\title{
Search for starless clumps in the ATLASGAL survey ${ }^{\star} \star \star$
}

\author{
J. Tackenberg ${ }^{1}$, H. Beuther ${ }^{1}$, T. Henning ${ }^{1}$, F. Schuller ${ }^{2}$, M. Wienen ${ }^{2}$, F. Motte ${ }^{7}$, F. Wyrowski², S. Bontemps ${ }^{3}$, \\ L. Bronfman ${ }^{4}$, K. Menten ${ }^{2}$, L. Testi ${ }^{5}$, and B. Lefloch ${ }^{6}$ \\ ${ }^{1}$ Max-Planck-Institut für Astronomie (MPIA), Königstuhl 17, 69117 Heidelberg, Germany \\ e-mail: tackenberg@mpia.de \\ 2 Max-Planck-Institut für Radioastronomie (MPIfR), Auf dem Hügel 69, 53121 Bonn, Germany \\ 3 OASU/LAB-UMR5804, CNRS/INSU, Université Bordeaux 1, 2 rue de l'Observatoire, 33270 Floirac, France \\ 4 Departamento de Astronomía, Universidad de Chile, Casilla 36-D, Santiago, Chile \\ 5 ESO, Karl-Schwarzschild Str. 2, 85748 Garching, Germany \\ ${ }^{6}$ Laboratoire d'Astrophysique de Grenoble, UMR 5571-CNRS, Université Joseph Fourier, Grenoble, France \\ 7 Laboratoire AIM, CEA/IRFU - CNRS/INSU - Université Paris Diderot, CEA-Saclay, 91191 Gif-sur-Yvette Cedex, France \\ Received 6 June 2011 / Accepted 16 January 2012
}

\section{ABSTRACT}

\begin{abstract}
Context. Understanding massive star formation requires comprehensive knowledge about the initial conditions of this process. The cradles of massive stars are believed to be located in dense and massive molecular clumps.

Aims. In this study, we present an unbiased sample of the earliest stages of massive star formation across $20 \mathrm{deg}^{2}$ of the sky.

Methods. Within the region $10^{\circ}<l<20^{\circ}$ and $|b|<1^{\circ}$, we search the ATLASGAL survey at $870 \mu \mathrm{m}$ for dense gas condensations. These clumps are carefully examined for indications of ongoing star formation using YSOs from the GLIMPSE source catalog as well as sources in the $24 \mu \mathrm{m}$ MIPSGAL images, to search for starless clumps. We calculate the column densities as well as the kinematic distances and masses for sources where the $v_{\mathrm{lsr}}$ is known from spectroscopic observations.

Results. Within the given region, we identify 210 starless clumps with peak column densities $>1 \times 10^{23} \mathrm{~cm}^{-2}$. In particular, we identify potential starless clumps on the other side of the Galaxy. The sizes of the clumps range between $0.1 \mathrm{pc}$ and $3 \mathrm{pc}$ with masses between a few tens of $M_{\odot}$ up to several ten thousands of $M_{\odot}$. Most of them may form massive stars, but in the $20 \mathrm{deg}^{2}$ area we only find 14 regions massive enough to form stars more massive than $20 M_{\odot}$ and 3 regions with the potential to form stars more massive than $40 M_{\odot}$. The slope of the high-mass tail of the clump mass function for clumps on the near side of the Galaxy is $\alpha=2.2$ and, therefore, Salpeter-like. We estimate the lifetime of the most massive starless clumps to be $(6 \pm 5) \times 10^{4} \mathrm{yr}$.

Conclusions. The sample offers a uniform selection of starless clumps. In the large area surveyed, we only find a few potential precursors of stars in the excess of $40 M_{\odot}$. It appears that the lifetime of these clumps is somewhat shorter than their free-fall times, although both values agree within the errors. In addition, these are ideal objects for detailed studies and follow-up observations.
\end{abstract}

Key words. ISM: clouds - stars: formation - surveys - stars: massive - stars: distances - Galaxy: fundamental parameters

\section{Introduction}

The understanding of high-mass star formation has made tremendous progress within the last decade (cf. reviews by Zinnecker \& Yorke 2007; Beuther et al. 2007). Nevertheless, there is still no consistent scenario describing how massive stars form, nor is the impact of massive stars on their environment fully understood. They undoubtedly play a crucial role throughout their whole lifetime on the physical and chemical structure of the interstellar medium, the evolution of stellar clusters, and even of entire galaxies. Therefore, massive star formation needs to be understood if we wish to make progress in our understanding of galaxy evolution.

Independent of the actual high-mass star formation scenario (e.g. Keto 2003; McKee \& Tan 2003; Bonnell \& Bate 2005; Commerçon et al. 2011), there is agreement that the most massive stars form in clusters. Therefore, it is probable that we can

\footnotetext{
* The catalog (Full Table 3) is only available in electronic form at the CDS via anonymous ftp to cdsarc.u-strasbg.fr (130.79.128.5) or via

http://cdsarc.u-strasbg.fr/viz-bin/qcat?]/A+A/540/A113

$\star \star$ Appendices and Fig. 12 are available in electronic form at

http://www . aanda.org
}

detect an initial stage of a high-mass gas clump without any star formation signatures.

The discovery of infrared-dark clouds (IRDCs) with ISO, MSX, and Spitzer data provided an interesting sample of objects with which to characterize the earliest stages of massive star formation (e.g. Perault et al. 1996; Carey et al. 1998). The systematic study of IRDCs provided potential precursors of massive stars and allowed the characterization of their physical and chemical parameters (e.g. Simon et al. 2006; Peretto \& Fuller 2009; Vasyunina et al. 2009, 2011). Although Peretto \& Fuller (2009) reported that more than $30 \%$ of the IRDCs have no IR counterparts at $24 \mu \mathrm{m}$, follow-up studies of IRDCs often revealed signs of ongoing star formation (Beuther \& Sridharan 2007; Cyganowski et al. 2008; Vasyunina et al. 2011).

All IRDC studies are biased by the variation of the background, foreground confusion, and extinction caused by variations in the dust properties. Longward of $200 \mu \mathrm{m}$, the thermal emission from dust grains in IRDCs is optically thin and can be measured at $\mathrm{mm}$ and sub-mm wavelengths (Hildebrand 1983). This can be used to obtain extinction-independent mass measurements of the cold gas inside these objects.

Until recently, there were no available systematic surveys of the Galactic plane directly tracing the cold dust associated with molecular clumps. Now, the Bolocam Galactic Plane Survey 
(BGPS, Aguirre et al. 2011) at $1.1 \mathrm{~mm}$, as well as the APEX Telescope Large Area Survey of the GALaxy (ATLASGAL, Schuller et al. 2009) at $870 \mu \mathrm{m}$, offer surveys of the Galactic plane's cold dust. Only ATLASGAL covers the full range $l=$ $-60^{\circ}$ to $60^{\circ}$ of the inner Galactic plane at $19^{\prime \prime}$ resolution.

In this paper, we present a compilation of clumps of high column density, located in a region of Galactic longitude $10^{\circ}<$ $l<20^{\circ}$ and latitude $-1^{\circ}<b<1^{\circ}$, showing no signs of star formation. To confirm their starless nature, we carefully examined each clump for GLIMPSE (Benjamin et al. 2003) and MIPSGAL $24 \mu \mathrm{m}$ (Carey et al. 2009) sources, either of which would indicate that star formation had already started. The column density threshold we imposed on our survey is $1 \times 10^{23} \mathrm{~cm}^{-2}$.

After a short description of the surveys we employed (Sect. 2), we describe the individual steps of the classification and its limitations in Sect. 3. In Sect. 4, we present both the results of the classification and the direct clump properties and discuss the clump column densities. Using the ammonia velocities given in Wienen et al. (2012), we derived distances to $\sim 71 \%$ of the clumps (Sect. 5). Section 6 presents the clump masses and the clump mass function. Next we estimated the lifetimes of starless clumps (Sect. 7). In Sect. 8, we discuss our results and compare them to other surveys (Sect. 8.3). Our conclusions, in Sect. 9, summarizes the main results of this work and provides an outlook to future work.

\section{Employed data}

All data for this study were taken from large surveys, most of them publicly available. Clumps were identified by searching for continuum peaks at $870 \mu \mathrm{m}$ in the ATLASGAL survey (Schuller et al. 2009) and then classified using both the GLIMPSE point source catalog and MIPSGAL $24 \mu \mathrm{m}$ images.

In contrast to most other searches for massive prestellar clumps of high column density using extinction maps (Simon et al. 2006; Peretto \& Fuller 2009; Kainulainen et al. 2011), we used emission at $870 \mu \mathrm{m}$ as a tracer of cold dust. The APEX telescope large area survey of the Galaxy (ATLASGAL, Schuller et al. 2009) is a systematic survey of the Galactic plane at $870 \mu \mathrm{m}$ with LABOCA (Siringo et al. 2009). Its beam size is 19.2", the pixel size in the maps is $6^{\prime \prime}$, and the average rms noise of the selected maps is below $50 \mathrm{mJy}$. To obtain a statistically meaningful sample, we covered $20 \mathrm{deg}^{2}$ on the sky, the region of Galactic longitude $10^{\circ}<l<20^{\circ}$ and Galactic latitude $|b|<1^{\circ}$.

As described in Sect. 3.2, we extracted young stellar objects from the GLIMPSE I Spring '07 catalog (Benjamin et al. 2003). Among other criteria, the GLIMPSE source catalog requires a minimum flux of $0.6 \mathrm{mJy}, 0.4 \mathrm{mJy}, 2 \mathrm{mJy}$, or $10 \mathrm{mJy}$ in either the $3.8,4.5,5.8$, or $8.0 \mu \mathrm{m}$ band, respectively, for a source to be taken into account. For the region given, we investigated more than 5.6 million GLIMPSE sources. The IRAC/Spitzer pointing accuracy is better than $1^{\prime \prime}$ and the pixel resolution is $0.6^{\prime \prime}$. As an additional tracer of ongoing star formation, we used the MIPSGAL $24 \mu \mathrm{m}$ survey (Carey et al. 2009). The rms noise of the MIPSGAL images is $\sim 0.67 \mathrm{mJy}$ and its resolution is $6^{\prime \prime}$.

\section{Classification}

The naming of clumps in the literature refers to various physical objects. Research groups working on high- and low-mass star formation have different naming schemes for the objects named clumps and cores, including the sub-categories starless and prestellar (Enoch et al. 2008). An often used nomenclature tries to bind things to physical properties, denoting gravitationally bound objects "cores" and unbound objects "clumps" (Chabrier \& Hennebelle 2010). In this paper, we use the term clumps for all emission peaks revealed by the CLUMPFIND algorithm (Williams et al. 1994). Typically, these are massive and large enough to form massive clusters. As shown in Fig. 5, typical sizes derived from the effective radii of these clumps range from $18^{\prime \prime}$ to $70^{\prime \prime}$. These can either be bound or unbound systems, but are assumed to be coherent in lbv space (Galactic longitude and latitude, and radial velocity, thus distance, Williams et al. 2000). In the remainder of this paper, clumps are called starless if they host no mid-IR tracers of ongoing star formation. Nevertheless, as mentioned in Sects. 1 and 3.3.3, many IRDCs not hosting $24 \mu \mathrm{m}$ sources have been shown to host star formation using other tracers such as $\mathrm{SiO}$ emission. In this study, we cannot completely rule out the presence of star formation, but only present starless clump candidates. In this context, the MALT90 survey (Foster et al. 2011) will improve future classifications.

\subsection{Clump extraction}

To identify starless clumps, we first employed the CLUMPFIND algorithm by Williams et al. (1994) to search for dust condensations. It has been reported that CLUMPFIND is less reliable in very crowded regions (Kainulainen et al. 2009) and that the extracted clump parameters strongly depend on the distance (Smith et al. 2008). The second point is unavoidable in observed data and is discussed further in Sect. 6.2. Nevertheless, we are interested in column-density peak positions and the associated fluxes/masses, which CLUMPFIND can extract reliably. Pineda et al. (2009) demonstrated that the exponent of the derived mass function is not very sensitive to the chosen step size.

We set the lowest detection level to $6 \sigma$, or $0.3 \mathrm{Jy}$. The additional thresholds, $0.3,0.4,0.5,0.7,0.9,1.3,1.8,2.5$, 4, and $7 \mathrm{Jy}$, were chosen (1) to account for the degree of variation relative to the actual emission level and (2) to trace the structures recognized by observers. The use of non-constant intervals for the various emission levels in CLUMPFIND prevents bright clumps from being artificially sub-divided because of brightness changes that are very small relative to the flux level of the clump.

To test the robustness of the chosen thresholds, we compared the integrated clump fluxes of our clump extraction to classical $3 \sigma$ spaced thresholds as proposed in Kainulainen et al. (2009). The two right columns of Fig. 2 compare the flux distributions along the lines plotted in the left column. The upper row shows our clump definition, the lower row shows the clumps of a pure $3 \sigma$ spacing of the contour levels. While some regions do not differ at all, e.g. as shown by the right-most column of Fig. 2, the additional contours in the evenly spaced situation can subdivide large clumps into a number of smaller ones, shown by the middle column of Fig. 2.

While flux level spacings that are not bound to some objective criteria introduce subjectivity, Fig. 2 shows that the chosen levels trace structures that we call clumps. In addition, we compared the fluxes of our clumps to the fluxes of the $3 \sigma$ extraction and could not find an excess of bright clumps, as might have been expected. Furthermore, we compared our CLUMPFIND sources to sources found by Contreras (priv. comm.) using SExtractor as described in Schuller et al. (2009). For fluxes above our thresholds, almost all sources identified by Contreras had a counterpart within our clumps with matching peak fluxes. In addition to this, the comparison shows that we identified smaller fragments of lower mass, and the integrated fluxes in our 
catalog are lower than the integrated fluxes of the corresponding SExtractor clumps. This assures us that we do not produce sources with artificially high fluxes.

As we aim to study sites of high-mass star formation, clumps with a peak flux of less than $0.5 \mathrm{Jy}$, corresponding to a column density of $1 \times 10^{23} \mathrm{~cm}^{-2}$, are ignored in the following. (For a further discussion of the derivation of column densities, we refer to Sect. 4.2.)

To motivate this threshold, we used the Orion nebula cluster (ONC). Its stellar mass is about $1800 M_{\odot}$ (Hillenbrand \& Hartmann 1998). To be consistent with estimates carried out in Sect. 7.1, we assumed a star formation efficiency of $30 \%$ and, therefore, estimated the initial gas mass of the ONC to be $6000 M_{\odot}$. As the cluster has dispersed over its lifetime, we set its initial radius to the typical radius we found for our most massive clumps, hence 0.7 pc (cf. Sect. 7.1). With the assumptions made and a spherically symmetric mass distribution, the initial peak column density in the $\mathrm{ONC}$ has been $1.8 \times 10^{23} \mathrm{~cm}^{-2}$, or $0.6 \mathrm{~g} \mathrm{~cm}^{-3}$. This agrees with the theoretical values found by Krumholz \& McKee (2008). To avoid fragmentation in highmass star formation, they require column densities of $1 \mathrm{~g} \mathrm{~cm}^{-2}$, or $3 \times 10^{23} \mathrm{~cm}^{-2}$.

\subsection{Identification of starless clumps}

Although it is unclear whether high- or low-mass stars form first, starless clumps should not host young stellar objects (class I sources, YSOs). To identify clumps hosting YSOs, we searched the GLIMPSE source catalog for stars with colors similar to known YSOs and compared those to our clumps. To do so, we followed the classification given by Gutermuth et al. (2008). We used additional color criteria, given in Gutermuth et al. (2008) as well, to reject contaminating extragalactic sources and AGNs "that masquerade as bona fide YSOs". Afterwards we selected objects obeying the following IRAC criteria:

$$
\begin{aligned}
& {[4.5]-[5.8]>1.0 \text { OR }} \\
& ([4.5]-[5.8]>0.7 \text { AND }[3.6]-[4.5]>0.7)
\end{aligned}
$$

In addition, we required a source to be detected at $8 \mu \mathrm{m}$. The identified YSOs were directly compared to the clumps and their extensions according to CLUMPFIND using the CLUMPFIND maps produced. If a YSO is located on a clump (in projection), the clump was considered as star forming and is ignored in the following. Nevertheless, at the onset of star formation, sources may be too cold to be detectable in the GLIMPSE bands, but show weak $24 \mu \mathrm{m}$ emission. Unfortunately, no MIPSGAL $24 \mu \mathrm{m}$ point source catalog has been published. Therefore, we used the STARFINDER algorithm by Diolaiti et al. (2000) to search the $24 \mu \mathrm{m}$ MIPSGAL images for point sources. To avoid misidentifications we only extracted stars with a detection better than $7 \sigma$. Again, clumps with a $24 \mu \mathrm{m}$ source were assumed to host stellar activity. In a last step, all remaining clumps were classified by visual inspection. Here the main focus was on $24 \mu \mathrm{m}$ objects that had not been identified by STARFINDER. A schematic summary of the classification is given in Fig. 1. Parts of M17, in which MIPSGAL is saturated due to extended emission, were omitted as well as a few additional regions. Exact positions of omitted regions are listed in Table B.1.

\subsection{Limitations and observational biases}

Although the visual verification of the classification ensures a maximum reliability, technical limitations of the data sets

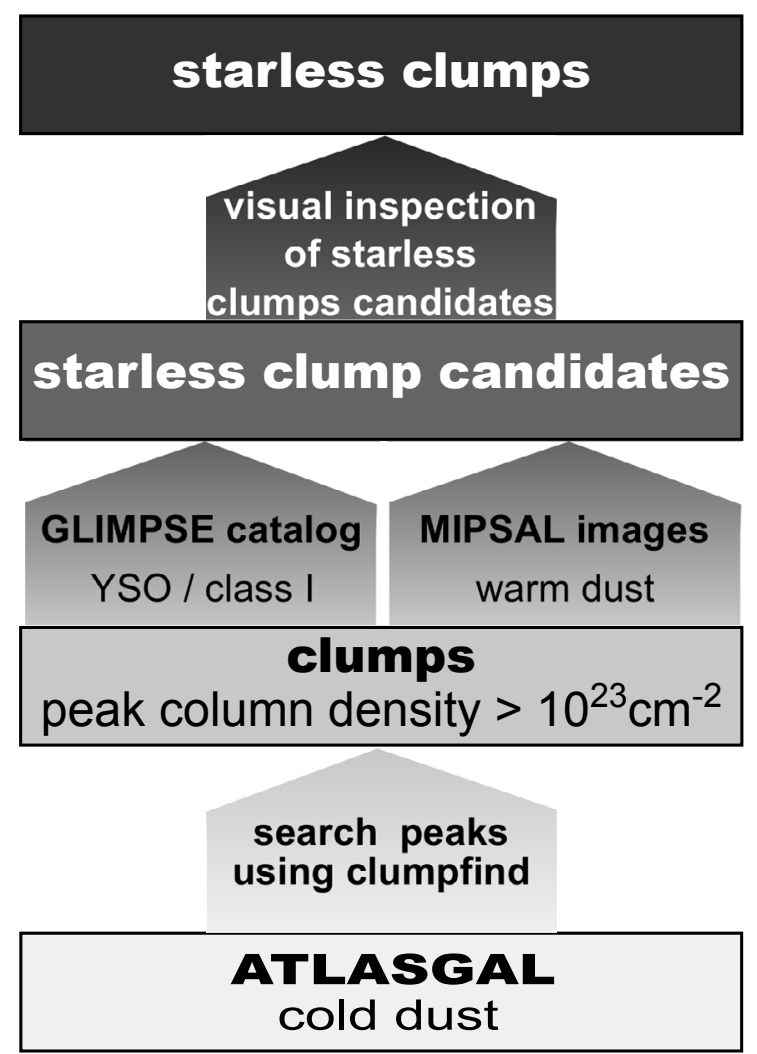

Fig. 1. Schematic visualization of classification.

impose various biases. To point out the limitations of this study, next we carefully discuss the biases.

\subsubsection{ATLASGAL and clump finding limitations}

The spatial limitations of ATLASGAL vary with the distance and are discussed in detail in Sect. 6.2. The flux threshold for the clump extraction was chosen to be $\sim 6 \sigma$ or $0.3 \mathrm{Jy}$, and the higher thresholds were chosen to trace clearly recognizable structures. These threshold spacings are larger than the estimated rms. Contours in steps of the noise level are less biased and would generate more substructure, hence clumps. However, noise would, more likely, generate artificial clumps as studied by Reid et al. (2010). The chosen peak flux threshold of $0.5 \mathrm{Jy} /$ beam corresponds to $1 \times 10^{23} \mathrm{~cm}^{-2}$. In the context of massive star formation, this provides a rough lower limit for potential regions of massive star formation (see Sect. 3.1). Sources for which the integrated flux is less than its peak flux are considered as artificial and 28 out of 929 sources were rejected.

\subsubsection{GLIMPSE catalog limitations}

The detection thresholds of GLIMPSE and MIPSGAL as well as the point source extraction from the GLIMPSE catalog and the color-color criteria for young sources described in Sect. 3.2 have a major impact on the classification. Gutermuth et al. (2008) included criteria to reject contaminating extragalactic sources, but AGB stars have similar colors to YSOs and obey the color-color criteria Gutermuth used to identify YSOs. Their contribution to the list of YSOs may be as high as $\sim 30 \%$ (Robitaille et al. 2008), rejecting potential starless clumps. Nevertheless, their likelihood of being projected onto a clump is significantly lower. Since we expect embedded YSOs 

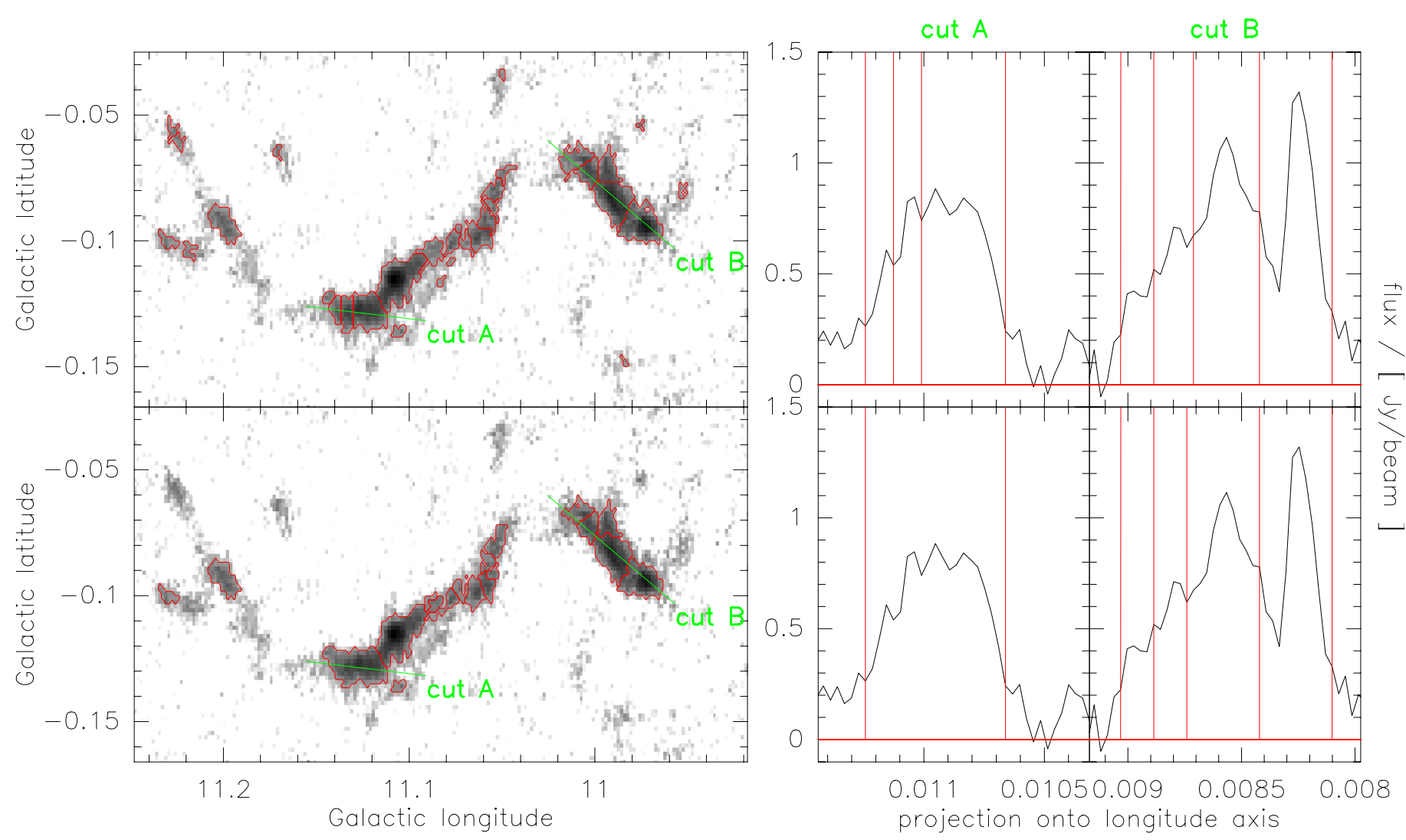

Fig. 2. The figures show the clump definition as used in this paper (bottom row), compared to "classical" $3 \sigma$ spacing contour levels (top row). While the left most column shows the ATLASGAL image of G11.11 with the clump definitions (red), the other columns show two profiles along the lines shown in the left panel ("cut A" and "cut B"). The red lines indicate the clump borders.

to have detectable $24 \mu \mathrm{m}$ flux for which we will check again, the given sensitivity limits of the GLIMPSE source catalog do not influence the population of starless clumps. Additional sources in the list of YSOs as well as chance alignments could lead to an artificial rejection of clumps, but will not produce artificial starless clumps.

\subsubsection{MIPSGAL $24 \mu \mathrm{m}$ limitations}

The situation is different for the $24 \mu \mathrm{m}$ MIPSGAL images. Here the sensitivity limit is the key parameter and sources hidden in the rms can lead to misidentifications of starless clumps. The brightness of the faintest sources still detectable varies over the images with respect to their surroundings, but for the visual inspection method we estimated it to be $\sim 1 \mathrm{mJy}$. This is slightly smaller than the $2 \mathrm{mJy}$ level for a $3 \sigma$ detection given in Carey et al. (2009).

For sources hidden in the dust, one may assume that all flux gets re-emitted by the dust producing a black body spectrum. From this, one can estimate the integrated luminosity of the internal source. Since the faint $24 \mu \mathrm{m}$ sources in question are not detected at GLIMPSE wavelengths, we used both the GLIMPSE and MIPSGAL detection limits to construct a SED of three data points at $3.8 \mu \mathrm{m}, 8.0 \mu \mathrm{m}$, and $24 \mu \mathrm{m}$ with $0.6 \mathrm{mJy}, 10.0 \mathrm{mJy}$, and $2.0 \mathrm{mJy}$, respectively. Using near- and mid-IR dust opacities from Draine \& Lee (1984), we fitted a black body spectrum to the SED, with an integrated luminosity of $1.1 L_{\odot}$ at $3 \mathrm{kpc}$, or $27 L_{\odot}$ at $15 \mathrm{kpc}$. These luminosities correspond to main sequence stars of $1.1 M_{\odot}$ or $2.1 M_{\odot}$ (Siess et al. 2000). Krumholz et al. (2007) found that accretion luminosities in massive star formation reach several hundred solar luminosities very early on, excluding that a massive collapsing core could be hidden in the dust. Furthermore, there have been observations of objects that may form high-mass stars, but are not yet that luminous (Beuther \& Steinacker 2007; Bontemps et al. 2010a; Motte et al. 2010, Ragan et al., in prep.). Nevertheless, their luminosities are still higher (on the order of several $10 L_{\odot}$ ) than our detection limit for $24 \mu \mathrm{m}$ fluxes on the far side of the Galaxy. Therefore, only low-mass objects can be hidden.

Observations using the Herschel satellite have shown that some $24 \mu \mathrm{m}$ dark regions, hence starless clumps already show $70 \mu \mathrm{m}$ emission (Beuther et al. 2010; Wilcock et al. 2011). As discussed in Henning et al. (2010), these sources may be either starless or protostellar. Similarly, Motte et al. (2007) and Russeil et al. (2010) find MSX and $24 \mu \mathrm{m}$ dark cores, driving SiO outflows. Although Motte et al. (2007) and Russeil et al. (2010) are less sensitive at $24 \mu \mathrm{m}$, future studies will need to disentangle this situation.

\subsection{Verification of classification by comparison to other tracers and studies}

To test the classification, a comparison with other tracers and catalogs is helpful.

Similar to Gutermuth et al. (2008), Robitaille et al. (2008) identified Intrinsically Red Sources (R08 in the following) by applying color-color criteria to the Spitzer GLIMPSE catalog. Both sets of color criteria differ and, in addition to a large number of common sources, both catalogs also identify different sources. We take these different identifications as statistical variations that set the approximate uncertainties in the different catalogs. If we now compare the population of starless clumps to 


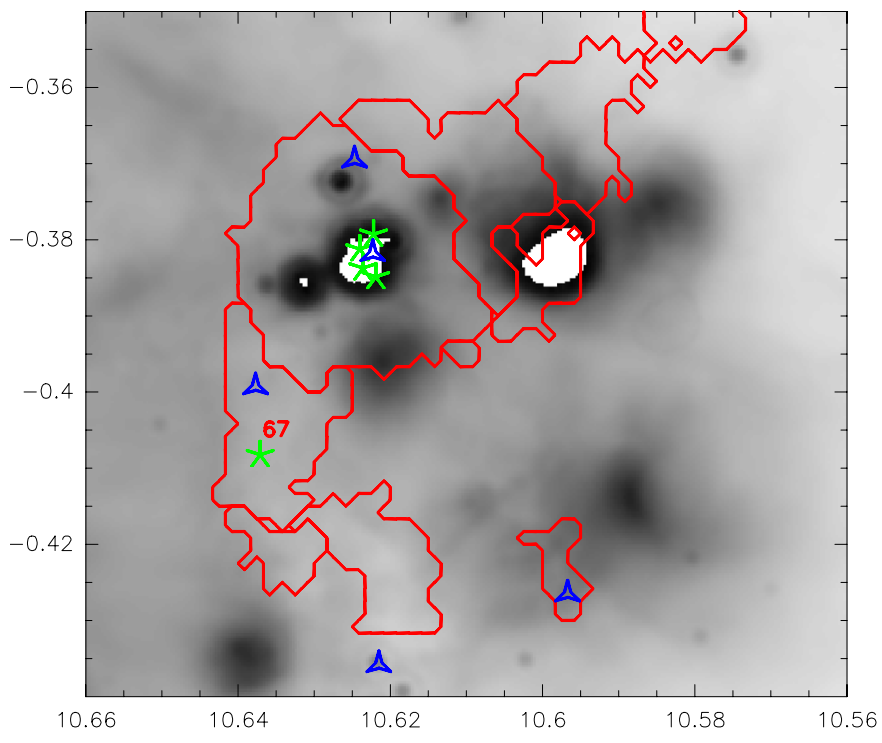

Fig. 3. Clump 67 overplotted on a MIPSGAL $24 \mu \mathrm{m}$ image. The clump definition are the red solid lines. Overplotted green asterisks are HII regions (Purcell \& Hoare 2010), and blue triangles are GLIMPSE Red Sources (Robitaille et al. 2008).

the Red Sources given in RO8, this gives us a feeling for the classification statistics. As it turns out, only two clumps that we identified as starless have a Spitzer Red Source. With knowledge of its position, we have been able to associate the R08 source in clump 67 with a peak in the $24 \mu \mathrm{m}$ image (see Fig. 3 ). The other Red Source is supposed to be in clump 1216, at a ridge of bright continuous emission. This hampers the identification and we cannot identify a $24 \mu \mathrm{m}$ counter part. Therefore, it is unclear to us whether this source is still very young.

Another test of our classification is to check the clumps for additional tracers of star formation. HII regions are a wellaccepted tracer of ongoing massive-star formation and several surveys have searched the Galactic plane systematically. In this context, CORNISH (Purcell \& Hoare 2010), a Galactic plane survey at $5 \mathrm{GHz}$ with the VLA in B configuration, identified more than 600 HII regions in our region of study. With their high spatial resolution, matches can be made unambiguously. We found Hir regions on only three of our clumps. In clump 67 (shown in Fig. 3) and clump 87, no $24 \mu \mathrm{m}$ source is in the vicinity of the $\mathrm{cm}$ emission peak. This suggests that star formation is already taking place, but so embedded that (almost) no light can escape. However, clump 505 has a very bright $24 \mu \mathrm{m}$ source at the edge of the clump. This might power the Hir region, which is offset by $11^{\prime \prime}$ towards the emission peak of clump 505 .

A comparison to the Green Bank Telescope HII Region Survey (GBT HRDS, Bania et al. 2010) and the Red MSX Source Survey (RMS, Hoare et al. 2004; Mottram et al. 2011) did not discover any matches.

In summary, since only three incorrect classifications have been found, all tests confirm our classification and establish its credibility. For consistency reasons, we flagged clump 67 as star forming, but kept the other two sources in our sample.

Furthermore, both pointed Herschel observations (e.g. EPOS, Krause et al., in prep.; Ragan et al., in prep.) and the Herschel Galactic plane survey HiGal (Molinari et al. 2010) revealed a new population of very young sources, detectable at $70 \mu \mathrm{m}$, but yet dark at $24 \mu \mathrm{m}$. Comparing the embedded protostars found in Henning et al. (2010) to the starless clumps we found in G11.11, we conclude that one out of six starless clumps harbors an embedded source, that is invisible at $24 \mu \mathrm{m}$. This suggests that not all clumps presented here will be starless at $70 \mu \mathrm{m}$. Future studies will need to clarify the Herschel view of our starless clumps.

\section{Distance independent results}

Using CLUMPFIND, we therefore extracted 901 clumps with peak column densities above our threshold of $1 \times 10^{23} \mathrm{~cm}^{2}$. We found that 291 clumps have a Spitzer counterpart classified as YSO using the Gutermuth criteria. For 238 objects, STARFINDER identified a $24 \mu \mathrm{m}$ (point) source in the MIPSGAL images, which had no YSO inside. During the visual inspection of the remaining 372 clumps, 103 additional $24 \mu \mathrm{m}$ sources were found, while 59 clumps were found to be partially or completely saturated in the MIPSGAL $24 \mu \mathrm{m}$ images. Therefore, 210 clumps, or $\sim 23 \%$, show no signs of a heating source with the data employed. These can be considered as starless.

The large number of visually identified $24 \mu \mathrm{m}$ sources show that this step was crucial for a reliable source catalog. Unfortunately, $24 \mu \mathrm{m}$ point sources are often hidden in the unsteady background emission, hence algorithmic point source extraction is unable to distinguish the weakest sources. The positions of the starless clumps are shown in a three color image, Fig. 4, and full details are given in Table 3. These clumps build a sample of potential starless clumps. As we show, most of them can be considered as massive.

\subsection{Results based on the classification}

It can be seen from Fig. 12 that almost all of the clumps are embedded in larger structures (cf. Schuller et al. 2009). These form filaments with lower density gas, indicated by the $3 \sigma$ contour. Only a few clumps seem to be isolated.

The majority of the gas is concentrated towards the Galactic plane, with a small offset towards negative latitudes. Enhanced concentrations of gas/clumps are visible towards known regions, mainly W31, W33, M17, M16, and W39 (from west to east).

A study to identify IRDCs, solely based on Spitzer $8 \mu \mathrm{m}$ extinction, was conducted by Peretto \& Fuller (2009). They found the fraction of starless IRDCs to be $32 \%$. This is similar to the fraction of $23 \%$ found within this work. Nevertheless, as pointed out in Peretto \& Fuller (2010), the detection of column densities via Spitzer $8 \mu \mathrm{m}$ extinction breaks down at column densities larger than $\sim 1 \times 10^{23} \mathrm{~cm}^{-2}$, which we require as minimum column density in our study. In addition, extinction is very unlikely to be observed on the far side of the Galaxy. Therefore, more than a quarter of the complexes have no Peretto \& Fuller (2009) IRDC close by and are a completely new sample, which is likely at the far side of the Galaxy.

The CLUMPFIND algorithm calculates the effective radius of the clumps by equating the area of a theoretical circular clump to the sum of the pixels. Results for the clump radii are shown in black in Fig. 5. The clump radii range between 10" and 40", with an average radius of $18^{\prime \prime}$.

\subsection{Column densities}

The fluxes at the peak positions of the clumps can be used to derive a beam-averaged peak column density. Within their large $\mathrm{NH}_{3}$ survey, Wienen et al. (2012) measured the rotation temperature of 15 of our starless clumps directly. Both the mean and 


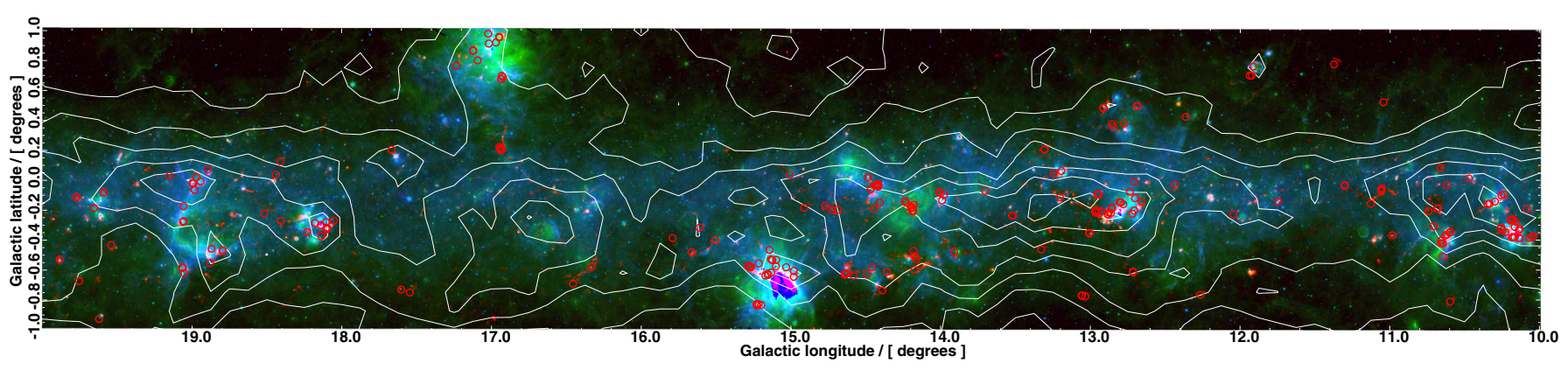

Fig. 4. RGB image of the Galactic plane with Galactic latitude $l=10^{\circ}$ to $20^{\circ}$ using GLIMPSE $8 \mu \mathrm{m}$, MIPSGAL $24 \mu \mathrm{m}$, and ATLASGAL $870 \mu \mathrm{m}$, respectively. Overplotted are CO contours from Dame et al. (2001). Starless cores are indicated as circles.

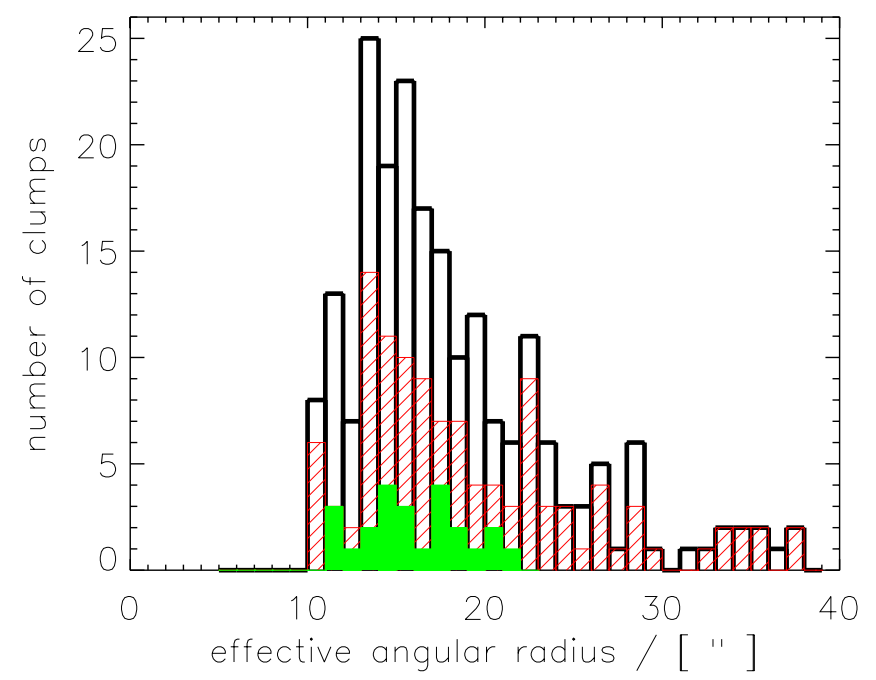

Fig. 5. Histogram of the effective radius derived by CLUMPFIND for all clumps (black), for clumps with IRDC connection (hatched red area), only, and for clumps without any IRDC indication (solid green area).

median temperature are $\mathrm{T}=15 \mathrm{~K}$ at these peak positions. This is in agreement with temperature estimates for IRDCs (Sridharan et al. 2005; Pillai et al. 2006; Vasyunina et al. 2011; Peretto et al. 2010). Since we required all clumps to be devoid of $24 \mu \mathrm{m}$ emission, we assumed that all our clumps have similar temperatures. We calculated the column density of the gas via

$N_{\mathrm{H}_{2}}=\frac{R F_{\lambda}}{B_{\lambda}(\lambda, T) m_{\mathrm{H}_{2}} K \Omega}$

for a gas-to-dust ratio of $R=100$, where $F_{\lambda}$ is the flux at the given wavelength, $B(\lambda, T)$ the blackbody radiation as a function of wavelength and temperature, $m_{\mathrm{H}_{2}}$ the mass per $\mathrm{H}_{2}$ molecule, and $\Omega$ the beam size. The mass absorption coefficient $\kappa=0.77 \mathrm{~cm}^{2} \mathrm{~g}^{-1}$ is adopted from the values given in Hildebrand (1983) using a dust emissivity index $\beta=2$ and an emissivity at $250 \mu \mathrm{m}$ of $3.75 \times 10^{-4}$. This is consistent with the value for the diffuse ISM in Ossenkopf \& Henning (1994), a frequently used value for dark clouds. The calculated column densities for the starless clumps are given in Table 3. For intermediate volume densities of $10^{6} \mathrm{~cm}^{-3}$ and thin ice mantles, one can extrapolate $\kappa=1.85 \mathrm{~cm}^{2} \mathrm{~g}^{-1}$ from Ossenkopf \& Henning (1994), as, e.g., used by Schuller et al. (2009). Including their different assumption of the mean molecular weight for the ISM, column density estimates in Schuller et al. (2009) would be smaller by a factor of three.

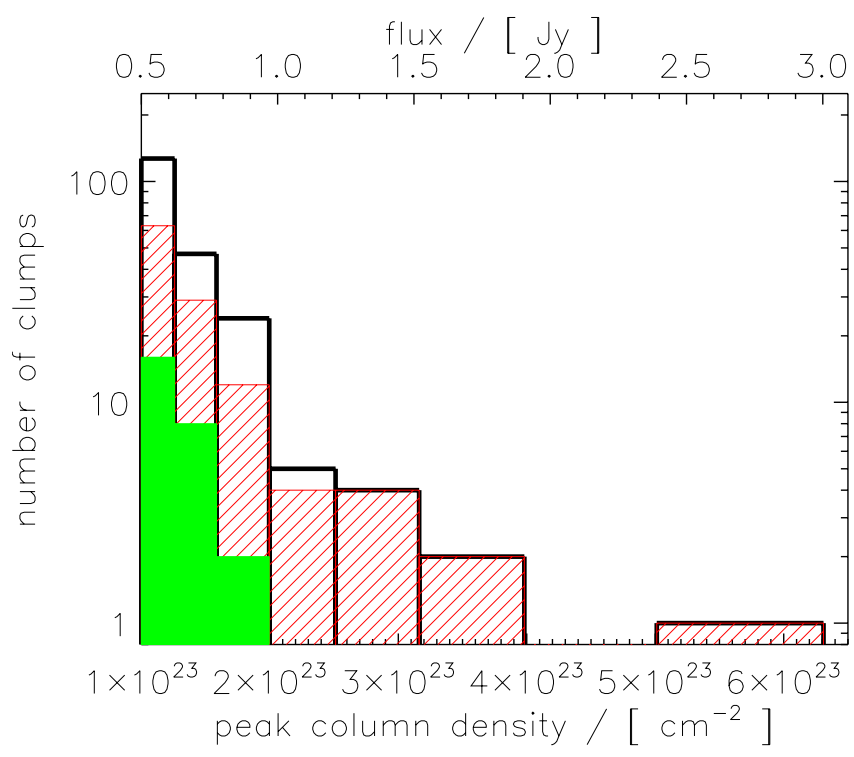

Fig. 6. Logarithmic histogram plot of the column density (lower $x$-axis) and flux (upper axis). While the black histogram represents the full sample of starless clumps, the hatched red and filled green histograms correspond to the near and far sample, respectively. (For details, cf. Sect. 5.)

The peak column densities vary only slightly. As shown in Fig. 6, 94\% of the starless clumps have column densities in the range between our survey threshold $1 \times 10^{23} \mathrm{~cm}^{-2}$ and $2 \times 10^{23} \mathrm{~cm}^{-2}$. Only 5 clumps, or $2 \%$, have a peak column density larger than $3 \times 10^{23} \mathrm{~cm}^{-2}$, which corresponds to $1 \mathrm{~g} \mathrm{~cm}^{-2}$. Nevertheless, the beam at a distance of $3 \mathrm{kpc}$ corresponds to $0.26 \mathrm{pc}$, hence is too large to resolve individual cores $(\sim 0.01-$ $0.1 \mathrm{pc})$. The given column densities are beam-averaged over large spatial scales and the actual peak column densities could be considerably larger. This effect preferentially reduces the column densities of clumps further away more significantly than those of nearby clumps, introducing an artificial difference between the clumps on this side and on the far side of the Galaxy. This difference is clearly illustrated by the red and green histograms in Fig. 6 for the near and far clumps, respectively (see Sect. 5).

To get a feeling for the small-scale peak column densities, Vasyunina et al. (2009) studied the effects of distance and telescope resolution onto the peak column density. First, they produced an artificial $r^{-1}$ density distribution grid of $2000 \mathrm{AU}$, or $0.01 \mathrm{pc}$, resolution. Secondly, they smoothed the grid with different Gaussian kernels to imitate observations with a 24" beam at different distances. They next compared the obtained column densities to the unsmoothed peak column densities and 
calculated from these ratios correction factors for different distances, which resemble the peak column density seen with a linear spatial resolution of $2000 \mathrm{AU}$. The correction factors applied for a distance of $\sim 2 \mathrm{kpc}$ start at around $\sim 17$, and go up to $\sim 40$ for distances around $4.5 \mathrm{kpc}$. Even assuming a minimal correction factor of $\sim 10$ and applying it to our sample, all clumps should contain smaller subregions of higher column densities, larger than $3 \times 10^{23} \mathrm{~cm}^{-2}$, following the Krumholz criterion for high-mass star formation. Nevertheless, this procedure cannot be applied to clumps at all distances. For clumps at the far side of the Galaxy in particular, the beam averages over several/many clumps and projection effects become more likely.

\section{Distances}

To determine additional physical parameters, in particular the size and the mass of the clumps, the distance is a major parameter. A Galactic rotation curve was utilized to determine distances from the clumps' radial velocities. In the following, only clumps with a distance estimate are discussed.

As the idea of this study was an unbiased survey of a large area of the sky with continuum data, a priori we have no information about the distances to the clumps found. To tackle this problem, we employed the Galactic rotation curve given in Reid et al. (2009). The necessary velocities are provided by Wienen et al. (2012). Wienen et al. (2012) conducted spectroscopic follow-up observations of $\mathrm{NH}_{3}$ towards bright peaks in the ATLASGAL survey. If no counterpart was found in Wienen et al. (2012), we used the $\mathrm{HCO}^{+}$survey of BGPS sources by Schlingman et al. (2011).

To maximize the number of clumps to which we could assign a velocity, all clumps that lie within the same lowest significant contour were assumed to be connected. With this assumption, we were able to assign to connected clumps the same velocities as their neighbors. Incorporating all information at hand, the velocities of 150 starless clumps, or $71 \%$, are known. The uncertainties in the velocities can be estimated by comparing the $\mathrm{NH}_{3}$ and $\mathrm{HCO}^{+}$velocities of clumps that have both measurements. We note that 134 of all clumps (not only starless clumps presented here) were observed by both Wienen et al. (2012) and Schlingman et al. (2011). The average difference between both velocity measurements is $0.5 \mathrm{~km} \mathrm{~s}^{-1}$, while their median difference is $0.3 \mathrm{~km} \mathrm{~s}^{-1}$, with $2.3 \mathrm{~km} \mathrm{~s}^{-1}$ being the largest difference. Therefore, we estimated the velocity uncertainties to be $0.5 \mathrm{~km} \mathrm{~s}^{-1}$.

Owing to the rotational structure and symmetry of the Galaxy, a Galactic rotation curve usually yields two distance solutions for a given direction and velocity. To solve this distance ambiguity, additional information or assumptions are required. While in studies of IRDCs it has often been argued that all sources lie at the near solution because their identification requires a bright mid-IR background, this argument could not be adopted here. The optically thin dust emission at $870 \mu \mathrm{m}$ instead allowed us to identify clumps across the entire Galaxy. Nevertheless, coincidence with an IRDC favors the near solution and we used the catalog of IRDCs given in Peretto \& Fuller (2009) to identify nearby objects within our sample. Although they cover a different column density range (for details see Sect. 4.1), considerable overlap can still be expected. During the visual inspection of the $24 \mu \mathrm{m}$ emission, additional dark patches connected to our clumps were identified and noted as IRDC. In the following, all clumps with an associated IRDC were assumed to be on the near side.

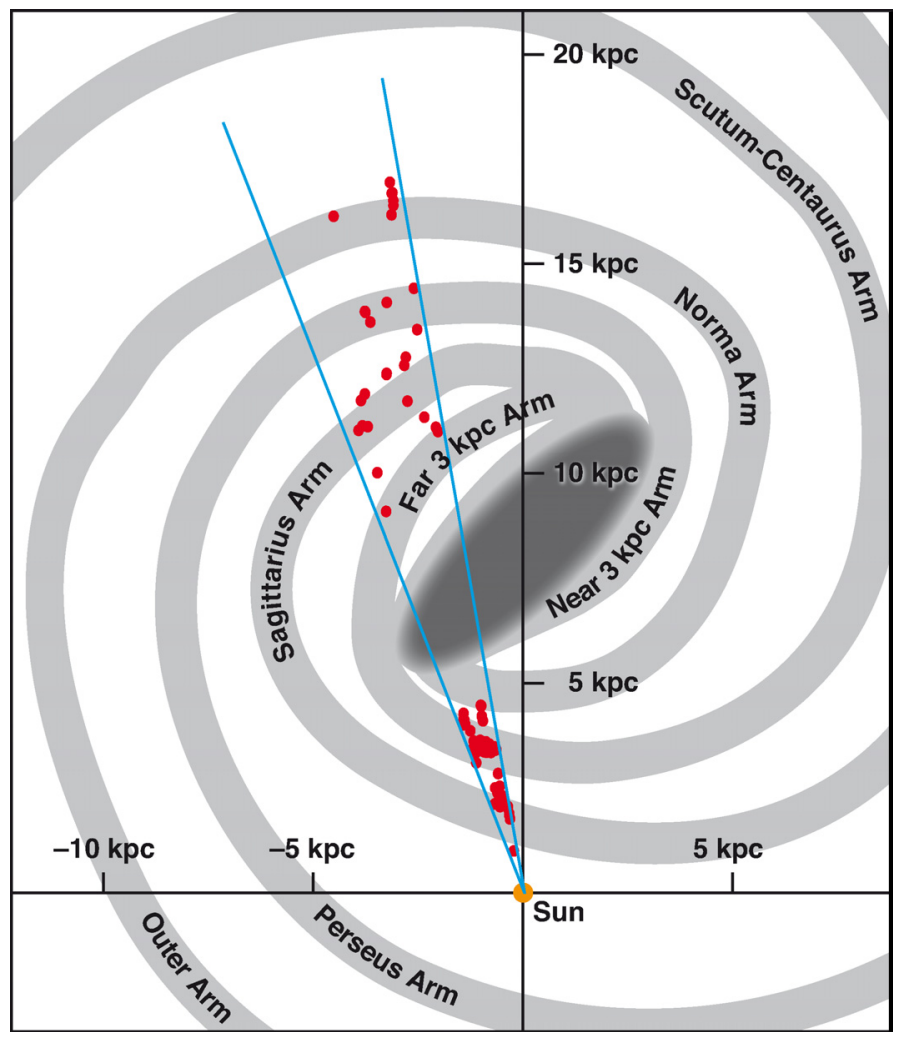

Fig. 7. Artist impression of face-on view of the Milky Way by R. Hurt (SSC-Caltech) / MPIA graphic. Plotted on top are the starless clumps presented here with the distance according to the distance flag in Table 3.

For 9 sources with velocities between $-5 \mathrm{~km} \mathrm{~s}^{-1}$ and $-1 \mathrm{~km} \mathrm{~s}^{-1}$, the rotation curve only allows the far solution, because their near solution is meaningless (it places the source in the outer Galaxy, while we looked in the opposite direction towards the inner Galaxy). The far solution places them in, or close to, the Norma arm at $\sim 17 \mathrm{kpc}$. However, as discussed in Dame \& Thaddeus (2008) and Green et al. (2011), the velocities could also place them in the near $3 \mathrm{kpc}$ arm at $\sim 5.2 \mathrm{kpc}$ distance. For consistency with the adopted Galactic rotation model, we prefer the Norma solution. Future studies of these clumps could also use $\mathrm{HI}$ self-absorption or ${ }^{13} \mathrm{CO}$ associations with well-known regions (Liszt et al. 1981) to better solve the distance ambiguity.

In summary, out of the 160 sources with velocity measurements 115 clumps are likely on the near side and 35 clumps are on the far side of the Galaxy (cf. Table 1 and Sect. 6.2). Only few starless clumps on the far side of the Galaxy have been known previously (Battersby et al. 2011), thus about a quarter of the sources are newly identified. Figure 7 shows the locations of the starless clumps within the Milky Way Galaxy. One notes a clear gap between $5 \mathrm{kpc}$ and $11 \mathrm{kpc}$ in the source distribution, which can be explained in several ways: (1) the elliptical orbits in the bulge of the Milky Way randomize its clouds' velocities and the rotation curve places them at random distances; (2) circular orbits close to the tangent point have very large $\frac{\mathrm{d}(\mathrm{dist})}{\mathrm{d} v}$, hence small errors in the velocities propagate into large distance offsets; (3) the majority of the cold gas is homogeneously distributed in a molecular ring around the Galactic center with $4 \mathrm{kpc}<R_{\mathrm{GC}}<8 \mathrm{kpc}$ (Solomon \& Rivolo 1989). Therefore, no clumps are expected outside that region. 
Table 1. Overview of typical clump properties for near and far clumps.

\begin{tabular}{lcccc}
\hline \hline Property reviewed & Near & Far & Only far & Estimated error near/far/only far \\
\hline Number of clumps & 115 & 26 & 9 & \\
Distance/[kpc] & 3.1 & 12.8 & 16.9 & 0.5 \\
Average effective radius / [pc] & 0.3 & 1.0 & 2.2 & $0.28 / 1.2 / 1.6$ \\
Average mass/[$\left.M_{\odot}\right]$ & 620 & 5560 & 26400 & factor of 4 \\
Median mass $/\left[M_{\odot}\right]$ & 320 & 4600 & 21400 & factor of 4 \\
Particle density $/\left[\mathrm{cm}^{-3}\right]$ & $1.0 \times 10^{5}$ & $2.3 \times 10^{4}$ & $1.3 \times 10^{4}$ & factor of 2 \\
\hline
\end{tabular}

Notes. The origin of the uncertainties is explained in Appendix A.

\section{Masses and clump mass function}

\subsection{Masses}

Assuming optically thin emission, the mass of these clumps can be calculated from the dust continuum emission via

$M_{\mathrm{gas}}=\frac{R d^{2} F_{\lambda}}{B_{\lambda}(\lambda, T) \kappa}$

where most of the parameters are the same as defined in Sect. 4.2, and $d$ is the distance. Therefore, the mass can only be calculated for sources with distance measurements. For completeness, the mass is calculated for both the near and far solutions produced by the distance ambiguity and listed in Table 3 . Figure 8 shows the calculated masses for both the near and far solutions. The solid line indicates the theoretical sensitivity limit of our source extraction. The uncertainties in the masses are discussed in Appendix A.

We expect the amount of dense gas per volume to be similar on both sides of the Galaxy. We can therefore conduct a consistency check by comparing the mass of the near and far population relative to the volume covered.

All clumps on the near side of the Galaxy have masses below $10^{4} M_{\odot}$ (cf. Fig. 8, black dots), while the maximum mass within the far-clumps is a factor of about three higher than the most massive clump on the near side. In addition, when looking at Fig. 8, the total number of near objects is clearly higher than the number of far objects. To make a quantitative comparison of both populations, we estimated the common sensitivity limit for distances between $10 \mathrm{kpc}$ and $15 \mathrm{kpc}$ to be $1 \times 10^{3} M_{\odot}$. We next calculated the volumes that we cover on the near and the far side using the scale height of $\sim 40 \mathrm{pc}$ given in Bronfman et al. (2000). Adding up all masses above the far detection threshold for the near range, $0 \mathrm{kpc}$ to $5 \mathrm{kpc}$, the total mass of clumps on the near side is $\sim 37000 M_{\odot}$. Doing the same for the far range $10 \mathrm{kpc}$ to $15 \mathrm{kpc}$ and normalizing it to the volume covered on the near side the total mass becomes $38000 M_{\odot}$. Both masses agree given this crude estimate, supporting the idea that, statistically, the allocation is reliable.

\subsection{Observational biases for clumps on the far side}

The change in resolution over the survey's depth affects the sensitivity and the recognition of substructure significantly. The $19.2^{\prime \prime}$ beam corresponds to $0.28 \mathrm{pc}$ linear spatial resolution at a distance of $3 \mathrm{kpc}$, and in contrast corresponds to $1.4 \mathrm{pc}$ at a distance of $15 \mathrm{kpc}$. While the distances vary by more than an order of magnitude, the angular sizes of the extracted clumps vary by only a factor 2-3 and show no correlation with distance (cf. Fig. 5). This results in an almost linear correlation between the physical size and the distance, which is shown in Fig. 9. In addition, a single, unresolved source would be 25 times fainter at

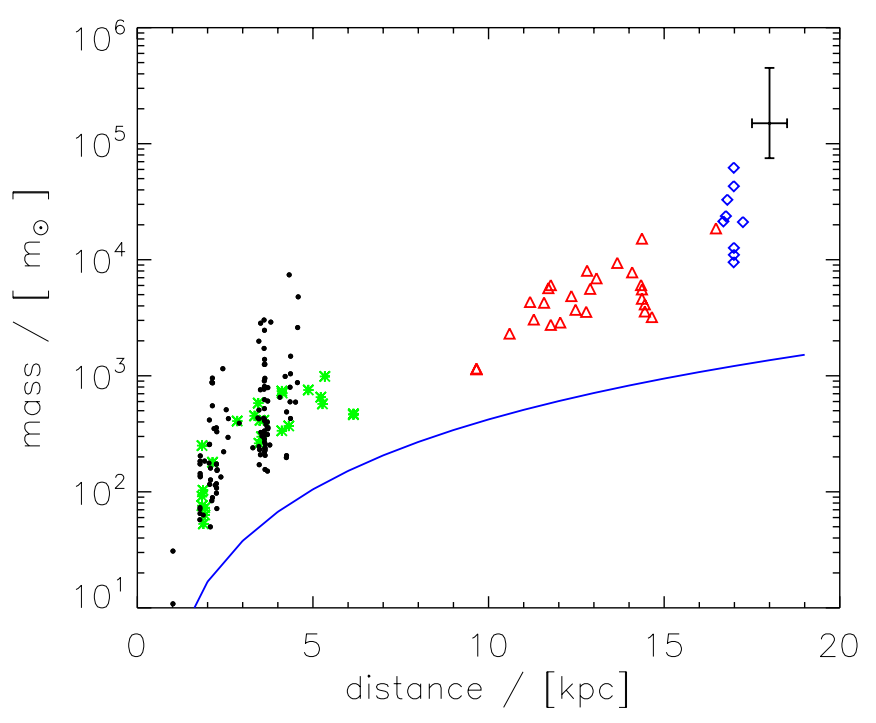

Fig. 8. The mass in solar masses is plotted over the distance in kpc. The solid line indicates the sensitivity/completeness limit of our clump extraction, which depends on the distance. The black filled dots represent sources with IRDC associations, and, therefore, the near distance is plotted. The red triangles represent the far solution of sources without an IRDC for which both a far and a near solution can be calculated; in addition, the green asterisks represent the corresponding near solutions. Blue diamonds show sources with such low velocities that only a single solution can be found in the given direction. The error bar in the top right corner indicates uncertainties of a factor of two for the masses and $0.5 \mathrm{kpc}$ for the distance.

$15 \mathrm{kpc}$ than at $3 \mathrm{kpc}$. As indicated by the solid line in Fig. 8, the completeness limit changes with distance. Both effects are studied in detail in the following.

Taking an ATLASGAL map of $3^{\circ} \times 2^{\circ}$, we re-extracted all clumps with CLUMPFIND using the same thresholds as explained above and calculated their masses assuming a generic distance of $3 \mathrm{kpc}$. In addition, we convolved the same map with a Gaussian profile, emulating a resolution of $96^{\prime \prime}$, reducing the resolution by a factor of 5 . This resembles the appearance of the same structure as seen at $15 \mathrm{kpc}$. Once again CLUMPFIND was used to search for clumps using the same parameters, but assuming a distance of $15 \mathrm{kpc}$ when determining the mass.

While $90 \%$ of the total mass was recovered in the lower resolution maps, the number of clumps extracted differed significantly. In the full resolution map, 328 clumps were extracted, whereas in the lower resolution map, only 20 clumps were found. This implies that structures, which can be resolved into several clumps on the near side, cannot be resolved on the far side and that their fluxes then add up.

The volumes covered at the two distances differ by a factor of about five. This would add to the probability of chance alignment 


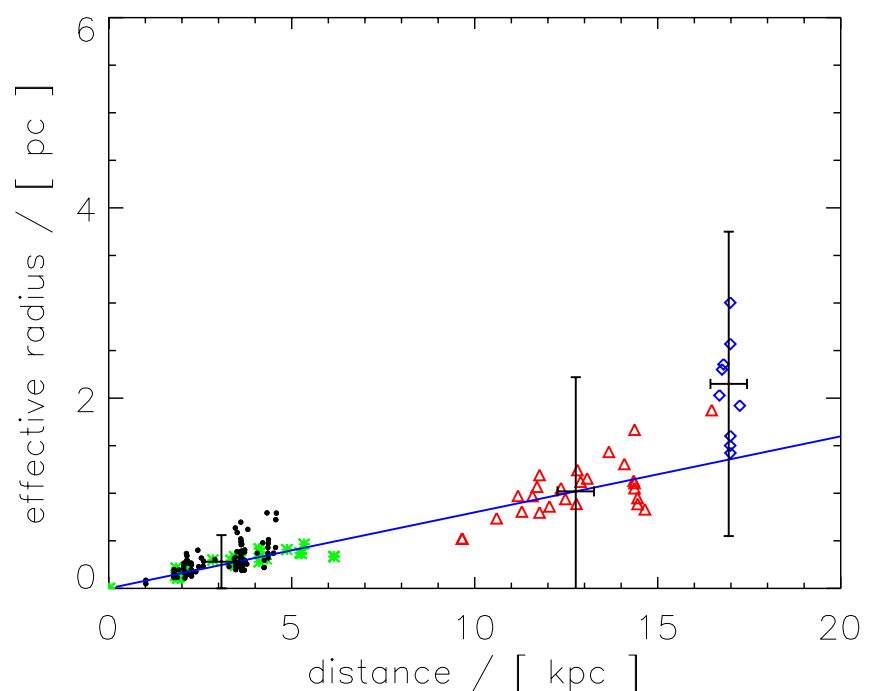

Fig. 9. Effective radius in pc over the distance. Colors and symbols are as in Fig. 8.

and since the dust emission is optically thin, several faint clumps within the same beam may add up and may become detectable.

Therefore, one should keep in mind that clumps discovered on the far side are slightly different types of objects.

\subsection{Clump-mass function (CMF)}

Mass distributions connected to stellar populations or star formation are very often compared to a power-law distribution, as first stated by Salpeter (1955) and discussed since then. The more recent study of Reid et al. (2010) even found that statistical errors allow the slope of (m)any sort(s) of astrophysical random distribution(s) to be fitted by a power-law with spectral indices similar to the Salpeter value of 2.35 within the errors. Nevertheless, a clump-mass function (CMF) is of great interest in connecting clumps to core- and star-formation efficiencies. To ensure that our data has sufficiently high quality statistics, we considered all 125 near clumps for a "local" CMF and the 27 far clumps for a CMF on the other side of the Galaxy. As shown in Fig. 8, the completeness limit for the near and far populations is lower than $100 M_{\odot}$ and $1000 M_{\odot}$, respectively. The black histogram in Fig. 10 represents the number distribution of the near clumps $\mathrm{d} N / \mathrm{d} M$. Owing to the completeness limits, we cannot rely on the low-mass end of the CMF below $100 M_{\odot}$, nor do we have sufficient statistics to determine the existence of a broken power-law distribution. We instead only consider the high-mass tail. The green line indicates a fit to the high-mass tail of the near-clump mass function, with a logarithmic slope of $\alpha=-2.2$. The red histogram is the far-clump mass function, fitted by the blue line of slope $\alpha=-2.6$. Neglecting the uncertainties on the data points, the uncertainties on the slopes calculated by the IDL routine "LINFIT" are 0.2 and 0.4 for the near- and far-clump mass functions, respectively. The thick bars at the bottom indicate the fitting range. If we expand the fitting range of the near population and include lower mass bins, the slope becomes immediately shallower. If we reduce the fitting range, the slope remains constant within the uncertainties. Therefore, we believe that the fitting range is reliable and the uncertainty is reasonable. For the far range, the situation is more difficult because of the smaller number of bins. An enlargement of the fitting range to smaller bins again reduces the slope dramatically. Including the next mass bin does not change the result significantly, but

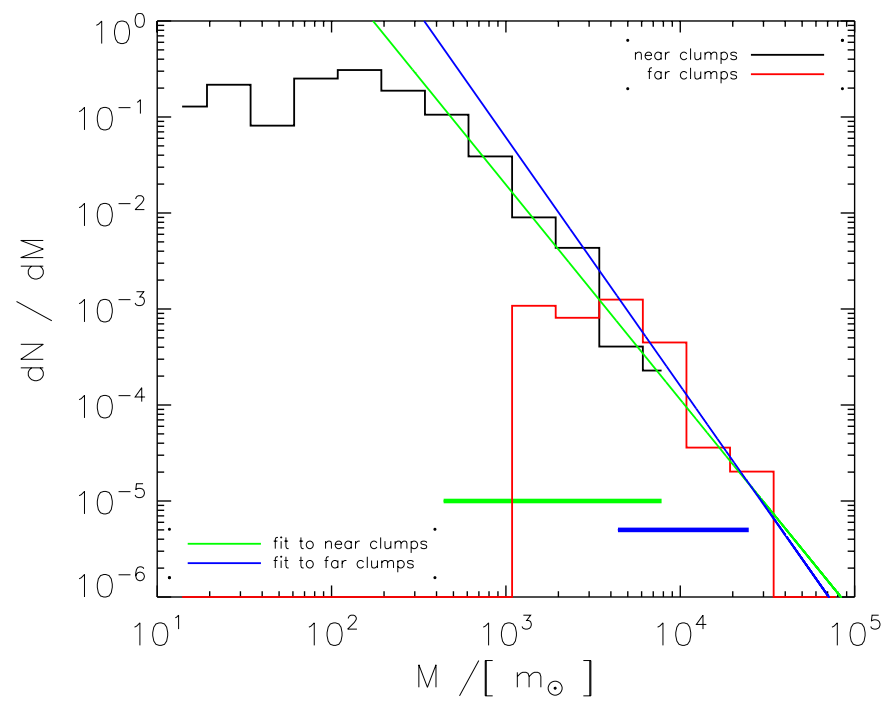

Fig. 10. Clump mass function with the near population plotted in black, the far population plotted in red. Both populations' high-mass tail have been fitted, with the fitting range indicated by the thick horizontal bars at the bottom. The power law indices are fitted to -2.2 and -2.6 for the near and far population, respectively.

including additional bins of lower mass would steepen the slope to -3.1. This number is almost within the errors and can be entirely explained by the lower quality statistics. Nevertheless, the fit to the far mass distribution should be interpreted with caution.

\section{Lifetimes}

As discussed in Sect. 6.2, clumps seen at the far side of the Galaxy are a mixture of clumps seen in chance-alignment or groupings that we would identify as several structures on the near side. To form a more consistent sample, in the following we only consider starless clumps that are identified on the near side.

\subsection{Mass of the most massive star}

To place constraints on the lifetime of starless clumps, we first need to estimate what clump mass is required so that the final cluster can house at least one massive star.

Star-formation efficiencies on scales from clumps to stars do not have a common value but a number of studies estimate that it is $23-50 \%$ (Chabrier \& Hennebelle 2010 and references therein). For high-mass star formation, the numbers are even more weakly constrained (5-50\%, cf. Krumholz et al. 2007; Kuiper et al. 2010).

Following the definition given in Williams et al. (2000) and Beuther et al. (2007), these clumps will most likely form entire clusters instead of single stars. To estimate the required clump mass to form a star of given mass, one must assume a gas-to-star formation efficiency and the initial mass function (IMF) of the cluster produced.

We assume the IMF of Kroupa (2001) and normalize it to the probability that at least one star with a mass higher than $20 M_{\odot}$ is formed. Integrating the normalized IMF over the expected mass range of stars, from 0.08 to $150 M_{\odot}$, the stellar mass of this cluster is on the order of $320 M_{\odot}$. With a star formation efficiency (SFE) of $30 \%$ (for details see next paragraph), we estimate the mass of a clump with the potential to form at least one star more 
Table 2. Lifetimes of starless clumps calculated for different sets of parameters.

\begin{tabular}{lcccc}
\hline \hline SFR & \multicolumn{2}{c}{ H83, diffuse ISM opacity } & \multicolumn{2}{c}{ OH94, cold dense opacities } \\
Mass of potential star & $20 M_{\odot}$ & $40 M_{\odot}$ & $20 M_{\odot}$ & $40 M_{\odot}$ \\
\hline Clump-mass threshold & $1065 M_{\odot}$ & $2960 M_{\odot}$ & $1065 M_{\odot}$ & $2960 M_{\odot}$ \\
Number of clumps above threshold & 14 & 3 & 6 & 1 \\
\hline SFR of $1 M_{\odot} / \mathrm{yr}$ & $3.3 \times 10^{5} \mathrm{yr}$ & $2.0 \times 10^{5} \mathrm{yr}$ & $1.4 \times 10^{5} \mathrm{yr}$ & $6.6 \times 10^{4} \mathrm{yr}$ \\
SFR of $3 M_{\odot} / \mathrm{yr}$ & $1.1 \times 10^{5} \mathrm{yr}$ & $6.6 \times 10^{4} \mathrm{yr}$ & $4.8 \times 10^{4} \mathrm{yr}$ & $2.2 \times 10^{4} \mathrm{yr}$ \\
SFR of $6 M_{\odot} / \mathrm{yr}$ & $5.5 \times 10^{4} \mathrm{yr}$ & $3.3 \times 10^{4} \mathrm{yr}$ & $2.4 \times 10^{4} \mathrm{yr}$ & $1.1 \times 10^{4} \mathrm{yr}$ \\
\hline
\end{tabular}

Notes. Estimates are calculated using different Milky Way star formation efficiencies for opacities for both the cold ISM (Hildebrand 1983, H83) and dense but cold regions (Ossenkopf \& Henning 1994, OH94).

massive than $20 M_{\odot}$ to be $10^{3} M_{\odot}$. A $3 \times 10^{3} M_{\odot}$ clump is required to form a $40 M_{\odot}$ star (see also Table 2).

Since the mass distribution follows a power law, the number of clumps with masses higher than a given threshold is very sensitive to that threshold. In the picture in which these clumps form entire clusters following the IMF, the estimate of the stellar cluster mass relative to the most massive star seems quite reliable. Nevertheless, the estimates of the SFE vary over a wide range (Lada \& Lada 2003; Alves et al. 2007; Johnston et al. 2009; Bontemps et al. 2010b). Here, we used the SFE given in Lada \& Lada (2003), Alves et al. (2007), and Bontemps et al. (2010b) of $30 \%$.

In the near sample derived across $20 \mathrm{deg}^{2}$ of the sky, with this estimate only 14 starless clumps have the potential to form stars more massive than $20 M_{\odot}$, and only 3 have the potential to form a $40 M_{\odot}$ star.

The MIPSGAL images of the 3 most massive clumps are shown in Fig. 11. As can be seen, none of the regions are isolated but all are connected to regions already containing $24 \mu \mathrm{m}$ sources. At the distances to these objects varying between $3.6 \mathrm{kpc}$ and $4.6 \mathrm{kpc}$, their effective radii become between $0.7 \mathrm{pc}$ and $0.8 \mathrm{pc}$. Therefore, their particle densities are not among the highest of our sample at only $4 \times 10^{4} \mathrm{~cm}^{-3}$ to $8 \times 10^{4} \mathrm{~cm}^{-3}$. Nevertheless, these are very interesting objects and very promising in the context of massive star formation.

\subsection{Lifetime of clumps}

Since there is no reason to believe that the starless clumps have ages that are correlated with those of other objects in this sample, the sample should span the entire age range expected for these clumps. If this is the case, the oldest clumps will start forming stars as new and similar clumps appear. Therefore, their lifetime can be calculated by comparing the number of these clumps identified to the number of massive stars formed.

To do so, we need to 1) extrapolate the number of starless clumps we would find in the entire Milky Way galaxy, and 2) estimate the number/fraction of massive stars formed every year:

1) We assume that most star-forming gas is distributed in a ring around the Galactic center, between $4 \mathrm{kpc}$ and $8 \mathrm{kpc}$ Galactocentric distance (Solomon \& Rivolo 1989) with a scale height of $40 \mathrm{pc}$ (Bronfman et al. 2000). Taking into account that we only consider clumps up to a distance of $5 \mathrm{kpc}$ from the Sun within the direction of the survey, and above the clump mass thresholds for $20 M_{\odot}$ and $40 M_{\odot}$ stars, for the whole Milky Way Galaxy, we expect to identify 1043 and 223 clumps, respectively.

2) To calculate the fraction of massive stars formed every year, one needs to assume a star-formation rate (SFR) for the Milky Way. The most recent publications suggest a star formation rate of around 1-2 $M_{\odot} / \mathrm{yr}$ (Robitaille \& Whitney 2010).

For a SFR of $1 M_{\odot} / y r$, the lifetimes of massive starless clumps become $3 \times 10^{5} \mathrm{yr}$ and $2 \times 10^{5} \mathrm{yr}$ calculated for all clumps more massive than $10^{3} M_{\odot}$, and $3 \times 10^{3} M_{\odot}$, respectively. For higher SFRs, the lifetimes become shorter in a linear fashion, e.g. for $6 M_{\odot} / \mathrm{yr}$ the lifetimes become $5.5 \times 10^{4} \mathrm{yr}$ and $3.3 \times 10^{4} \mathrm{yr}$, respectively. Lifetimes for different parameters are summarized in Table 2. As we discuss in Sect. 8.2, we estimate the lifetime of these objects to be $(6 \pm 5) \times 10^{4} \mathrm{yr}$.

\section{Discussion}

\subsection{Discussion of the clump-mass function}

The slope of the CMF for starless clumps found in this study, $\alpha=-2.2$, is similar to the value of the Salpeter IMF. Nonetheless, as we study clumps that will most likely host small clusters rather than individual stars, we do not propose a one-toone mapping to the IMF. We emphasize that not all clumps will eventually form clusters or even be transient objects. Another set of massive clumps was presented in Peretto \& Fuller (2009), including a subset of clumps without MIPS $24 \mu \mathrm{m}$ emission. They found the mass function of IRDCs to be similar to the CO clump mass distribution (Simon et al. 2006; Peretto \& Fuller 2010) with $\alpha=-1.7$. In addition, they used a derivative of CLUMPFIND to search their extinction maps for structures within the IRDCs. These fragments have a rather Salpeter-like slope (Rathborne et al. 2006; Peretto \& Fuller 2010) which is then similar to our result. Although the extinction method used by Peretto \& Fuller (2009) to calculate column densities of IRDCs is sensitive to a lower column density range than that of the starless clumps we present, the $870 \mu \mathrm{m}$ emission identifies objects similar to fragments Peretto \& Fuller (2009) find in IRDCs.

The studies of Williams et al. (2004), Reid \& Wilson (2005), and Beltrán et al. (2006) all targeted the more evolved high-mass protostellar objects. They found a common break in the CMF at $100 M_{\odot}$ and fit power laws to their high-mass end with exponents between -2.0 and -2.32 . Although we did not attempt to fit the exact break, a break point of $100 M_{\odot}$ or just above seems to be in good agreement with the starless CMF, but that clearly might be biased by the completeness limit close to $100 M_{\odot}$. However, the exponent of the near CMF agrees with all values within the errors. Thus, comparing our results to earlier studies of more evolved clumps shows that there is no evidence that the CMF of starless clumps is different from a CMF at later evolutionary stages.

Thus, we found that the exponent of the CMF for clumps at the far side of the Galaxy is larger than most other values. Although the uncertainty is larger and the difference could be explained by the errors, this trend might equally represent a general 

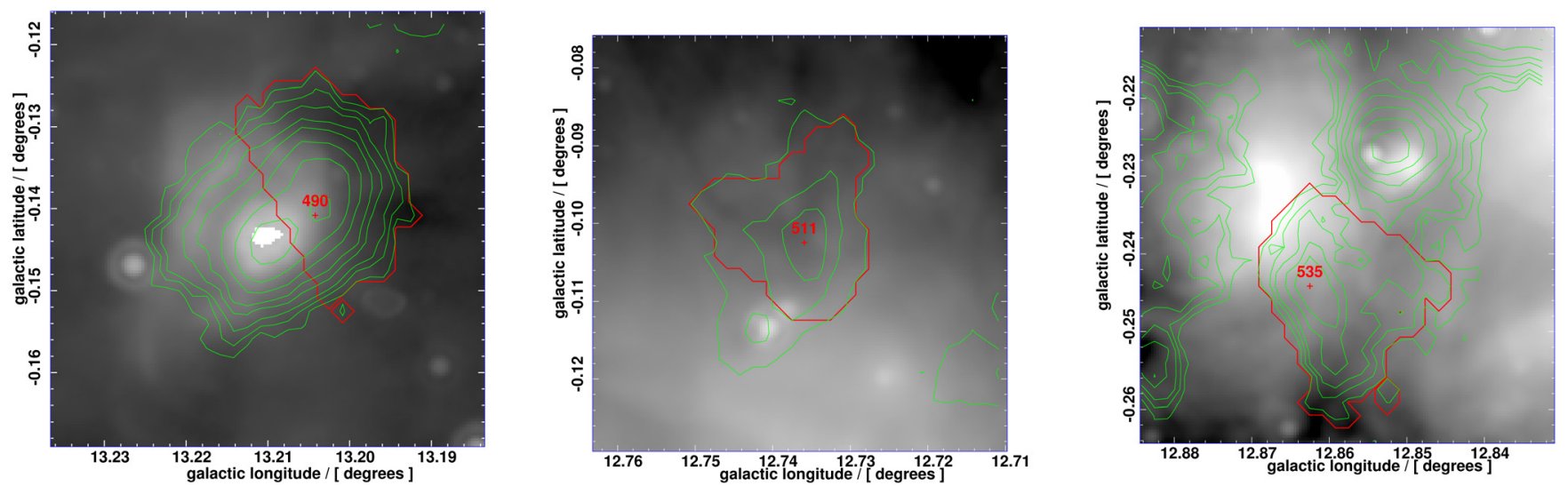

Fig. 11. MIPSGAL images of the three most massive starless clumps found in this survey, with (from left to right ) $7400 M_{\odot}, 4700 M_{\odot}$, and $3000 M_{\odot}$. Green contours are from ATLASGAL, red lines mark the boarders of the clumps identified by CLUMPFIND.

scale-dependent trend. Beltrán et al. (2006) distinguished between the populations at $d<2 \mathrm{kpc}$ and $d<6 \mathrm{kpc}$ and found that the exponent for the more clearly resolved population $<2 \mathrm{kpc}$ is shallower than for $d<6 \mathrm{kpc}$. Our far population is even more distant than their sample and the far CMF's exponent would continue the trend to steeper slopes. This could be interpreted as a general scale-dependent trend and rather a matter of resolution than of true structure.

Studies of the core mass function for low-mass star formation target significantly smaller objects. To have sufficient spatial resolution, they are typically chosen to be nearby. Nevertheless, whether they present starless cores or more evolved objects, many studies have measured power-law slopes close to the Salpeter value (Motte et al. 1998; Johnstone et al. 2000; Alves et al. 2007; Enoch et al. 2008).

\subsection{Discussion of the lifetimes}

The estimates of the lifetimes of starless clumps are based on the SFR of a given mass range and the number of clumps above a corresponding clump mass. Both estimates involve several assumptions, which may introduce errors.

Lifetimes are inversely proportional to the SFR. The SFR varies from $1 M_{\odot} / \mathrm{yr}$ (Robitaille \& Whitney 2010) to over $4 M_{\odot} / \mathrm{yr}$ (Diehl et al. 2006) to even larger values, but the most recent publications favor the smaller values. Nevertheless, our own survey looks at a region in the vicinity of the Galactic center for which both Rosolowsky et al. (2010) and Beuther et al. (2012) found that more dense gas is located within the inner $l<30^{\circ}$ of the Galactic plane. While most gas is concentrated within a molecular ring around the Galactic center at $4 \mathrm{kpc}<R<8 \mathrm{kpc}$ (Solomon \& Rivolo 1989), the ring does not seem homogeneous and the outer regions of that ring seem to contain less gas. This might indicate that the extrapolated numbers for the entire Galaxy might be higher than average and therefore require a higher SFR. This would reduce the lifetime estimate.

The lifetimes are also proportional to the number of clumps of given mass and when we use the extrapolated dust opacities from Ossenkopf \& Henning (1994) our clump masses decrease and the number of clumps above our threshold is lower. This, in turn, reduces the lifetimes. In addition, one should keep in mind that small number statistics are involved. If one of these objects turns out to already be star-forming, the estimated lifetimes are reduced, while correcting chance alignments of mid-IR sources has the opposite effect.

Another factor contributing to the uncertainties are the starformation efficiencies, which directly influence the mass thresholds themselves. As explained in Sect. 7.1, the number distribution decays as a power of the mass, which makes the lifetime estimates very sensitive to the clump mass thresholds. On the basis of the range of possible lifetimes shown in Table 2, we estimated the uncertainties to be one order of magnitude.

In summary, most effects seem to reduce the lifetimes. Based on these arguments, we estimate the lifetime of starless clumps to be on the order of $(6 \pm 5) \times 10^{4} \mathrm{yr}$.

For volume-averaged particle densities of $10^{5} \mathrm{~cm}^{-3}$, the freefall time becomes $\sim 1.6 \times 10^{5} \mathrm{yr}$, and lower densities increase this number. Therefore, the free-fall time is about a factor of two longer than the lifetime we found for starless clumps, but both agree within the errors.

In good agreement with previous studies (Motte et al. 2007; Hatchell \& Fuller 2008; Motte et al. 2010), Table 2 shows that the estimated lifetimes of more massive clumps are smaller. Accordingly, it was expected that the lifetimes we found for starless dense clumps are shorter than the $3 \times 10^{5} \mathrm{yr}$ found by Kirk et al. (2005) for low-mass cores.

An adequate comparison to the lifetime of high-mass starless clumps is difficult because only a few studies exist. Most deal with slightly different kinds of objects. For example, Motte et al. (2007) studied the nearby Cygnus X region and found cores that cover the same volume densities as our clumps, while their masses are significantly lower (see also Motte et al. 2010). However, they found clumps of similar mass to our objects but with lower volume densities. Using $\mathrm{SiO}$ as an additional tracer of star formation, Motte et al. (2007) did not find a single starless massive dense core in their sample. Therefore they proposed that the low-density starless clumps dynamically evolve into starforming massive dense cores. Their lifetime estimate of starless massive dense cores becomes $<10^{3} \mathrm{yr}$.

A similar study covering more similar entities both in density and mass by Russeil et al. (2010) found one starless clump, hence they derived a statistical lifetime of $\sim 1 \times 10^{4} \mathrm{yr}$. This agrees with our estimate, but at the lower edge of the error. The difference could also be caused by their inclusion of $\mathrm{SiO}$ as a tracer of star formation, which could reduce the number of starless clumps we found. 


\subsection{Comparison to other surveys}

\subsubsection{Comparison to the Bolocam Galactic Plane Survey (BGPS)}

The Bolocam Galactic Plane Survey (BGPS) performed a systematic study of the inner Galactic plane at $1.1 \mathrm{~mm}$. Although their rms across the region $10^{\circ}<l<20^{\circ}$ is $\sim 25 \mathrm{mJy} /$ beam, their overall $5 \sigma$ level is $0.4 \mathrm{Jy}$, at which they have a completeness level of $99 \%$. Their 0.4 Jy completeness threshold converts into a column density threshold of $\sim 6 \times 10^{22} \mathrm{~cm}^{-2}$ over their $31^{\prime \prime}$ beam when using the same assumptions as for our data, while their sensitivity translates into a rms of $64 \mathrm{mJy} /$ beam at $870 \mu \mathrm{m}$ and their beam width of $31^{\prime \prime}$. With this column density, they found 1211 sources on the same region as we studied here.

For that data set, Dunham et al. (2011) used different point source catalogs to search within all BGPS sources for midinfrared tracers of star formation activity. As resources they employed the Red Sources (R08, Robitaille et al. 2008), the RMS catalog, the catalog of extended green objects (EGOs, Cyganowski et al. 2008), and the full GLIMPSE catalog. The EGOs have no direct counterpart in this study, but trace shocked gas. To be truly starless, starless clumps should not contain EGOs.

Reviewing the Dunham et al. (2011) source catalog for the same region as surveyed in this study, we found that for $\sim 70 \%$ of the sources they found neither RMS sources, R08 sources, nor EGOs.

Following a similar approach to Dunham et al. (2011) and using the identical mid-IR tracers, the R08, RMS, and EGO catalogs, for the ATLASGAL clumps, we found R08 counterparts within 163 clumps, 39 RMS counterparts within clumps without R08 sources, and 9 matches among EGOs and clumps without the previous tracers. This leaves 719 , or $77 \%$, of our ATLASGAL clumps starless. Both fractions of infrared quiet clumps are significantly larger than those of Peretto \& Fuller (2009) $(32 \%)$ or what we found $(23 \%)$ including all tracers. Even without visual inspection but including MIPSGAL $24 \mu \mathrm{m}$ sources found by STARFINDER, we would have found only $42 \%$ of the clumps to be starless. These differences in our statistics from both of the latter studies can be explained by the superior sensitivity of the "by eye" source confirmation and the higher spatial resolution of the MIPSGAL survey over the MSX images.

\subsubsection{Comparison to Planck's Early Cold Cores}

That Planck's Early Cold Core Source List (ECC) (Planck Collaboration 2011) does not contain any cold sources in the region of the sky we surveyed here is apparently remarkable. However, the ECC contains only sources colder than $T<14 \mathrm{~K}$ averaged over Planck's beam sizes of several arc minutes. Owing to the high gas density and ongoing star formation as well as confusion, no sources are expected to be found within the Galactic plane (Planck Collaboration 2011).

\section{Conclusion and outlook}

\subsection{Conclusion}

For the first time, we have presented a complete and unbiased sample of high-mass starless clumps on $20 \mathrm{deg}^{2}$ of the sky. To concentrate on the actual potential precursors of massive stars, we imposed a minimum peak column density of $1 \times 10^{23} \mathrm{~cm}^{-2}$. From ATLASGAL, we extracted 901 clumps across the region $10^{\circ}<l<20^{\circ}$ of the Galactic plane. Using the GLIMPSE source catalog and MIPSGAL $24 \mu \mathrm{m}$ images, we compared clumps found at $870 \mu \mathrm{m}$ to near- and mid-infrared tracers of ongoing star formation.

Neglecting clumps that are saturated at MIPSGAL $24 \mu \mathrm{m}$, we identified 210 clumps, or $23 \%$, to be starless. Their effective radii range from $10^{\prime \prime}$ to $40^{\prime \prime}$, and most of the beamaveraged peak column densities are in the range $1-2 \times 10^{23} \mathrm{~cm}^{-2}$. Correcting the single-dish peak column densities to true peak column densities as discussed in Vasyunina et al. (2009) even suggests that all clumps should have peak column densities above the fragmentation threshold for massive star formation of $1 \mathrm{~cm}^{-2}$ proposed by Krumholz \& McKee (2008).

For $\sim 71 \%$ of the starless clumps, we had the velocities and were able to calculate their distance. If a clump is connected to an IRDC, then we assumed a distance on the near side. We found that about a quarter of the starless clumps lie on the far side of the Galaxy and were therefore previously unknown.

We found that the masses of starless clumps on the near side range from $10 M_{\odot}$ to $7500 M_{\odot}$, and that objects on the far side have masses between $1000 M_{\odot}$ to several $10000 M_{\odot}$. The different mass regimes are a consequence of our limited spatial resolution, biasing us towards detecting larger structures as a single clump on the far side, which we would resolve into several clumps on the near side. The mass distributions of the near and far populations could be fitted by power laws with slopes $\alpha=-2.2$ and $\alpha=-2.6$, respectively, and agree within the errors. This shows that the mass distribution of clump populations on the near and far side of the Galaxy do not differ significantly.

Drawn from the population of clumps on the near side, we found that only 14 objects are massive enough to form clusters with stars more massive than $20 M_{\odot}$. Only 3 starless clumps have the potential to form stars even more massive than $40 M_{\odot}$. We estimate the minimum clump mass required to form a cluster with a $20 M_{\odot}$ or $40 M_{\odot}$ star to be $10^{3} M_{\odot}$ or $3 \times 10^{3} M_{\odot}$, respectively. Since the star-formation efficiency used is an upper limit, these numbers are lower limits.

Extrapolating the numbers of massive starless clumps from our survey volume to the Milky Way Galaxy, we estimated the lifetime of the most massive starless clumps to be on the order of $(6 \pm 5) \times 10^{4} \mathrm{yr}$. To do so, we assumed a star formation rate of $1 M_{\odot}$ to $3 M_{\odot}$ per year for the entire Galaxy. We also discussed a possibly enhanced star formation activity within the surveyed volume and its implications for the assumed global star formation rate.

\subsection{Outlook}

In the future, we wish to extend our classification of the clumps to more evolved stages. To identify massive young stellar objects in particular, the Red MSX Source (RMS) survey will be used. Incorporating CORNISH (see Sect. 3.4) and RMS, in addition to GLIMPSE and MIPSGAL, we plan to establish an evolutionary sequence of the early stages of massive star formation. With this classification, we will be able to derive the relative timescales for the full sequence. Using the absolute timescale established in Sect. 7.2, we will be able to translate the relative into absolute timescales and compare these to earlier studies.

In addition, the objects that we have presented herein represent an ideal and unique sample for follow-up studies of the early stages of massive star formation. The upcoming HIGAL/Herschel survey (Molinari et al. 2010) will help to provide additional constraints on the nature of these clumps, but deep 
J. Tackenberg et al.: Starless clumps in ATLASGAL

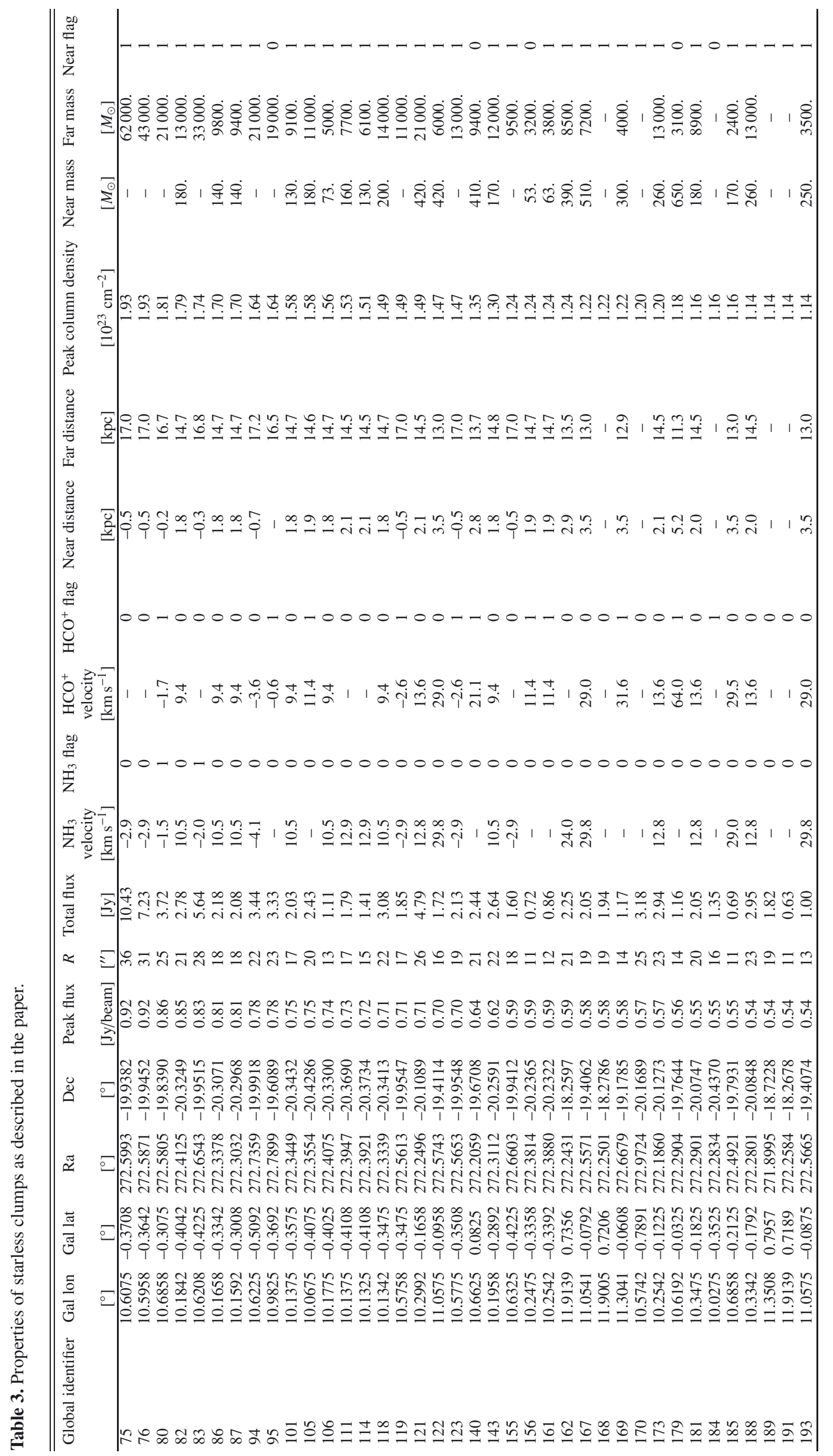

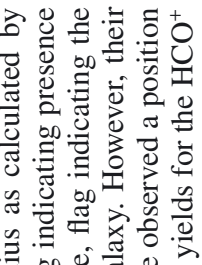
密

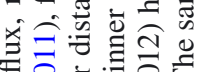

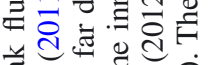

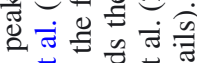

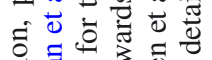
.ี. G5 5005 in

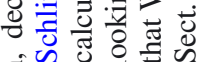

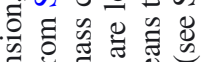

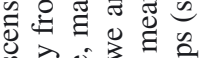

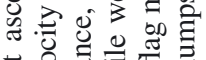

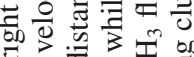

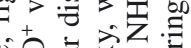

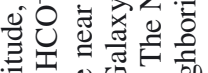
壱

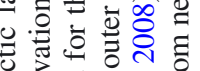

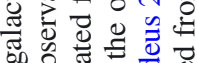

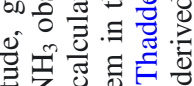

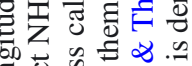

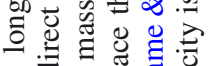
记

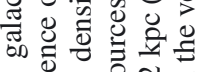
उ)

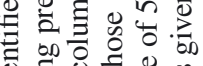

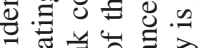

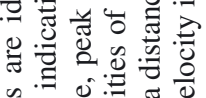

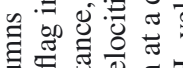

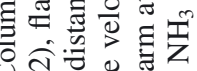

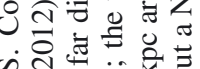

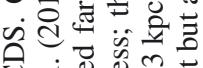

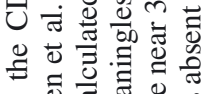

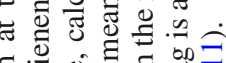

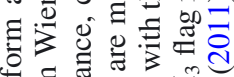
ह छ

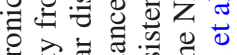

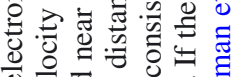

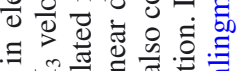

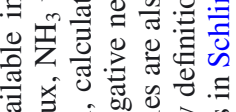

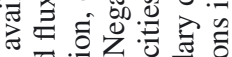
$\leadsto$ 焉记远

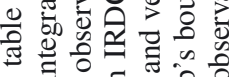

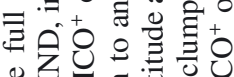

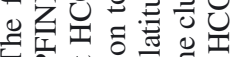

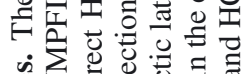

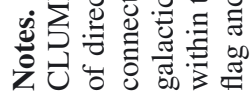


integrations with the Herschel $100 \mu \mathrm{m}$ band will be needed to uncover all embedded sources. In addition, the high resolution of ALMA will allow detailed studies of star-forming regions on the other side of the Galaxy.

Acknowledgements. We wish to thank the anonymous referee for her/his careful reading and comments, which substantially enhanced both the science and appearance of the paper. This publication is partially based on data acquired with the Atacama Pathfinder Experiment (APEX). APEX is a collaboration between the Max-Planck-Institut für Radioastronomie, the European Southern Observatory, and the Onsala Space Observatory. This work is based, in part, on observations made with the Spitzer Space Telescope, which is operated by the Jet Propulsion Laboratory, California Institute of Technology under a contract with NASA. This research has made use of the NASA/ IPAC Infrared Science Archive, which is operated by the Jet Propulsion Laboratory, California Institute of Technology, under contract with the National Aeronautics and Space Administration. L.B. acknowledges support from CONICYT projects FONDAP 15010003 and Basal PFB-06. J.T. is supported by the International Max Planck Research School (IMPRS) for Astronomy and Cosmic Physics.

\section{References}

Aguirre, J. E., Ginsburg, A. G., Dunham, M. K., et al. 2011, ApJS, 192, 4 Alves, J., Lombardi, M., \& Lada, C. J. 2007, A\&A, 462, L17

Bania, T. M., Anderson, L. D., Balser, D. S., \& Rood, R. T. 2010, ApJ, 718, L106

Battersby, C., Bally, J., Ginsburg, A., et al. 2011, A\&A, 535, A128

Beltrán, M. T., Brand, J., Cesaroni, R., et al. 2006, A\&A, 447, 221

Benjamin, R. A., Churchwell, E., Babler, B. L., et al. 2003, PASP, 115, 953

Beuther, H., \& Sridharan, T. K. 2007, ApJ, 668, 348

Beuther, H., \& Steinacker, J. 2007, ApJ, 656, L85

Beuther, H., Churchwell, E. B., McKee, C. F., \& Tan, J. C. 2007, Protostars and Planets V, 165

Beuther, H., Henning, T., Linz, H., et al. 2010, A\&A, 518, L78

Beuther, H., Tackenberg, J., Linz, H., et al. 2012, ApJ, 747, 43

Bonnell, I. A., \& Bate, M. R. 2005, MNRAS, 362, 915

Bontemps, S., André, P., Könyves, V., et al. 2010a, A\&A, 518, L85

Bontemps, S., Motte, F., Csengeri, T., \& Schneider, N. 2010b, A\&A, 524, A18

Bronfman, L., Casassus, S., May, J., \& Nyman, L. 2000, A\&A, 358, 521

Carey, S. J., Clark, F. O., Egan, M. P., et al. 1998, ApJ, 508, 721

Carey, S. J., Noriega-Crespo, A., Mizuno, D. R., et al. 2009, PASP, 121, 76

Chabrier, G., \& Hennebelle, P. 2010, ApJ, 725, L79

Commerçon, B., Hennebelle, P., \& Henning, T. 2011, ApJ, 742, L9

Cyganowski, C. J., Whitney, B. A., Holden, E., et al. 2008, AJ, 136, 2391

Dame, T. M., \& Thaddeus, P. 2008, ApJ, 683, L143

Dame, T. M., Hartmann, D., \& Thaddeus, P. 2001, ApJ, 547, 792

Diehl, R., Halloin, H., Kretschmer, K., et al. 2006, Nature, 439, 45

Diolaiti, E., Bendinelli, O., Bonaccini, D., et al. 2000, A\&AS, 147, 335

Draine, B. T., \& Lee, H. M. 1984, ApJ, 285, 89

Dunham, M. K., Robitaille, T. P., Evans, II, N. J., et al. 2011, ApJ, 731, 90

Enoch, M. L., Evans, II, N. J., Sargent, A. I., et al. 2008, ApJ, 684, 1240

Foster, J. B., Jackson, J. M., Barris, E., et al. 2011, ApJS, 197, 25

Green, J. A., Caswell, J. L., McClure-Griffiths, N. M., et al. 2011, ApJ, 733, 27

Gutermuth, R. A., Myers, P. C., Megeath, S. T., et al. 2008, ApJ, 674, 336

Hatchell, J., \& Fuller, G. A. 2008, A\&A, 482, 855

Henning, T., Linz, H., Krause, O., et al. 2010, A\&A, 518, L95

Hildebrand, R. H. 1983, QJRAS, 24, 267
Hillenbrand, L. A., \& Hartmann, L. W. 1998, ApJ, 492, 540

Hoare, M. G., Lumsden, S. L., Oudmaijer, R. D., et al. 2004, in Milky Way Surveys: The Structure and Evolution of our Galaxy, ed. D. Clemens, R. Shah, \& T. Brainerd, ASP Conf. Ser., 317, 156

Johnston, K. G., Shepherd, D. S., Aguirre, J. E., et al. 2009, ApJ, 707, 283

Johnstone, D., Wilson, C. D., Moriarty-Schieven, G., et al. 2000, ApJ, 545, 327

Kainulainen, J., Lada, C. J., Rathborne, J. M., \& Alves, J. F. 2009, A\&A, 497, 399

Kainulainen, J., Alves, J., Beuther, H., Henning, T., \& Schuller, F. 2011, A\&A, 536, A48

Keto, E. 2003, ApJ, 599, 1196

Kirk, J. M., Ward-Thompson, D., \& André, P. 2005, MNRAS, 360, 1506

Kroupa, P. 2001, MNRAS, 322, 231

Krumholz, M. R., \& McKee, C. F. 2008, Nature, 451, 1082

Krumholz, M. R., Klein, R. I., \& McKee, C. F. 2007, ApJ, 656, 959

Kuiper, R., Klahr, H., Beuther, H., \& Henning, T. 2010, ApJ, 722, 1556

Lada, C. J., \& Lada, E. A. 2003, ARA\&A, 41, 57

Liszt, H. S., Burton, W. B., \& Bania, T. M. 1981, ApJ, 246, 74

McKee, C. F., \& Tan, J. C. 2003, ApJ, 585, 850

Molinari, S., Swinyard, B., Bally, J., et al. 2010, PASP, 122, 314

Motte, F., Andre, P., \& Neri, R. 1998, A\&A, 336, 150

Motte, F., Bontemps, S., Schilke, P., et al. 2007, A\&A, 476, 1243

Motte, F., Zavagno, A., Bontemps, S., et al. 2010, A\&A, 518, L77

Mottram, J. C., Hoare, M. G., Urquhart, J. S., et al. 2011, A\&A, 525, A149

Ossenkopf, V., \& Henning, T. 1994, A\&A, 291, 943

Perault, M., Omont, A., Simon, G., et al. 1996, A\&A, 315, L165

Peretto, N., \& Fuller, G. A. 2009, A\&A, 505, 405

Peretto, N., \& Fuller, G. A. 2010, ApJ, 723, 555

Peretto, N., Fuller, G. A., Plume, R., et al. 2010, A\&A, 518, L98

Pillai, T., Wyrowski, F., Carey, S. J., \& Menten, K. M. 2006, A\&A, 450, 569

Pineda, J. E., Rosolowsky, E. W., \& Goodman, A. A. 2009, ApJ, 699, L134

Planck Collaboration 2011, A\&A, 536, A7

Purcell, C. R., \& Hoare, M. G. 2010, Highlights of Astronomy, 15, 781

Rathborne, J. M., Jackson, J. M., \& Simon, R. 2006, ApJ, 641, 389

Reid, M. A., \& Wilson, C. D. 2005, ApJ, 625, 891

Reid, M. J., Menten, K. M., Zheng, X. W., et al. 2009, ApJ, 700, 137

Reid, M. A., Wadsley, J., Petitclerc, N., \& Sills, A. 2010, ApJ, 719, 561

Robitaille, T. P., \& Whitney, B. A. 2010, ApJ, 710, L11

Robitaille, T. P., Meade, M. R., Babler, B. L., et al. 2008, AJ, 136, 2413

Rosolowsky, E., Dunham, M. K., Ginsburg, A., et al. 2010, ApJS, 188, 123

Russeil, D., Zavagno, A., Motte, F., et al. 2010, A\&A, 515, A55

Salpeter, E. E. 1955, ApJ, 121, 161

Schlingman, W. M., Shirley, Y. L., Schenk, D. E., et al. 2011, ApJS, 195, 14

Schuller, F., Menten, K. M., Contreras, Y., et al. 2009, A\&A, 504, 415

Siess, L., Dufour, E., \& Forestini, M. 2000, A\&A, 358, 593

Simon, R., Jackson, J. M., Rathborne, J. M., \& Chambers, E. T. 2006, ApJ, 639, 227

Siringo, G., Kreysa, E., Kovács, A., et al. 2009, A\&A, 497, 945

Smith, R. J., Clark, P. C., \& Bonnell, I. A. 2008, MNRAS, 391, 1091

Solomon, P. M. \& Rivolo, A. R. 1989, ApJ, 339, 919

Sridharan, T. K., Beuther, H., Saito, M., Wyrowski, F., \& Schilke, P. 2005, ApJ, 634, L57

Vasyunina, T., Linz, H., Henning, T., et al. 2009, A\&A, 499, 149

Vasyunina, T., Linz, H., Henning, T., et al. 2011, A\&A, 527, A88

Wienen, M., Wyrowski, F., Schuller, F., et al. 2012, A\&A, submitted

Wilcock, L. A., Kirk, J. M., Stamatellos, D., et al. 2011, A\&A, 526, A159

Williams, J. P., de Geus, E. J., \& Blitz, L. 1994, ApJ, 428, 693

Williams, J. P., Blitz, L., \& McKee, C. F. 2000, Protostars and Planets IV, 97

Williams, S. J., Fuller, G. A., \& Sridharan, T. K. 2004, A\&A, 417, 115

Zinnecker, H. \& Yorke, H. W. 2007, ARA\&A, 45, 481

Pages 15 to 36 are available in the electronic edition of the journal at http://www . aanda. org 
J. Tackenberg et al.: Starless clumps in ATLASGAL
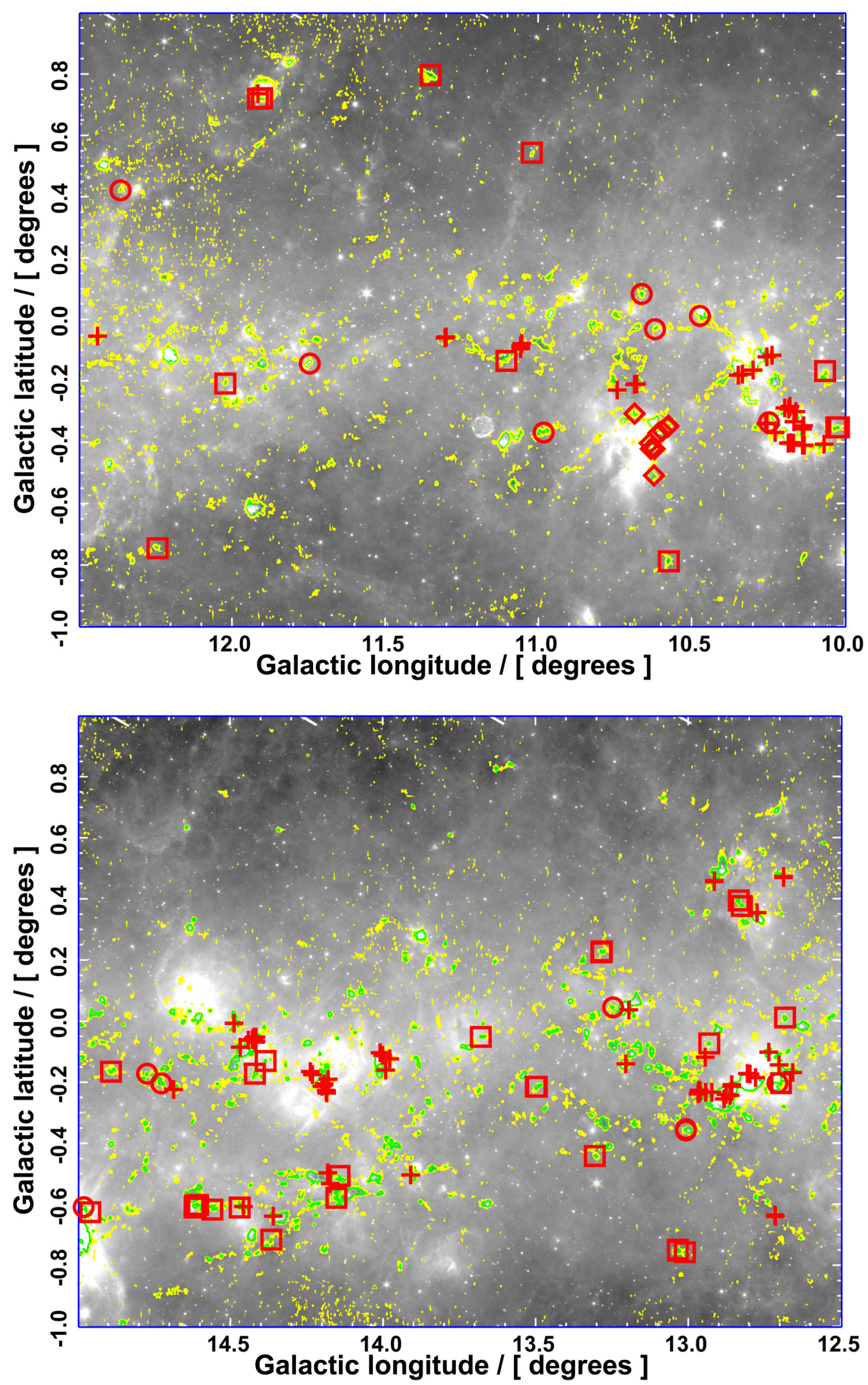

Fig. 12. ATLASGAL 3 and $6 \sigma$ contours in yellow and green, respectively, on top of a $24 \mu \mathrm{m}$ MIPSGAL image in logarithmic scale, with clumps plotted in overlay. Plus signs represent clumps for which the near solutions is assumed while circles are clumps with far solution assumed. For clumps plotted with a diamond only the far solution exists, while for sources with a box no velocity information is present. White stripes at the edges are artifacts from the MIPSGAL coverage. 

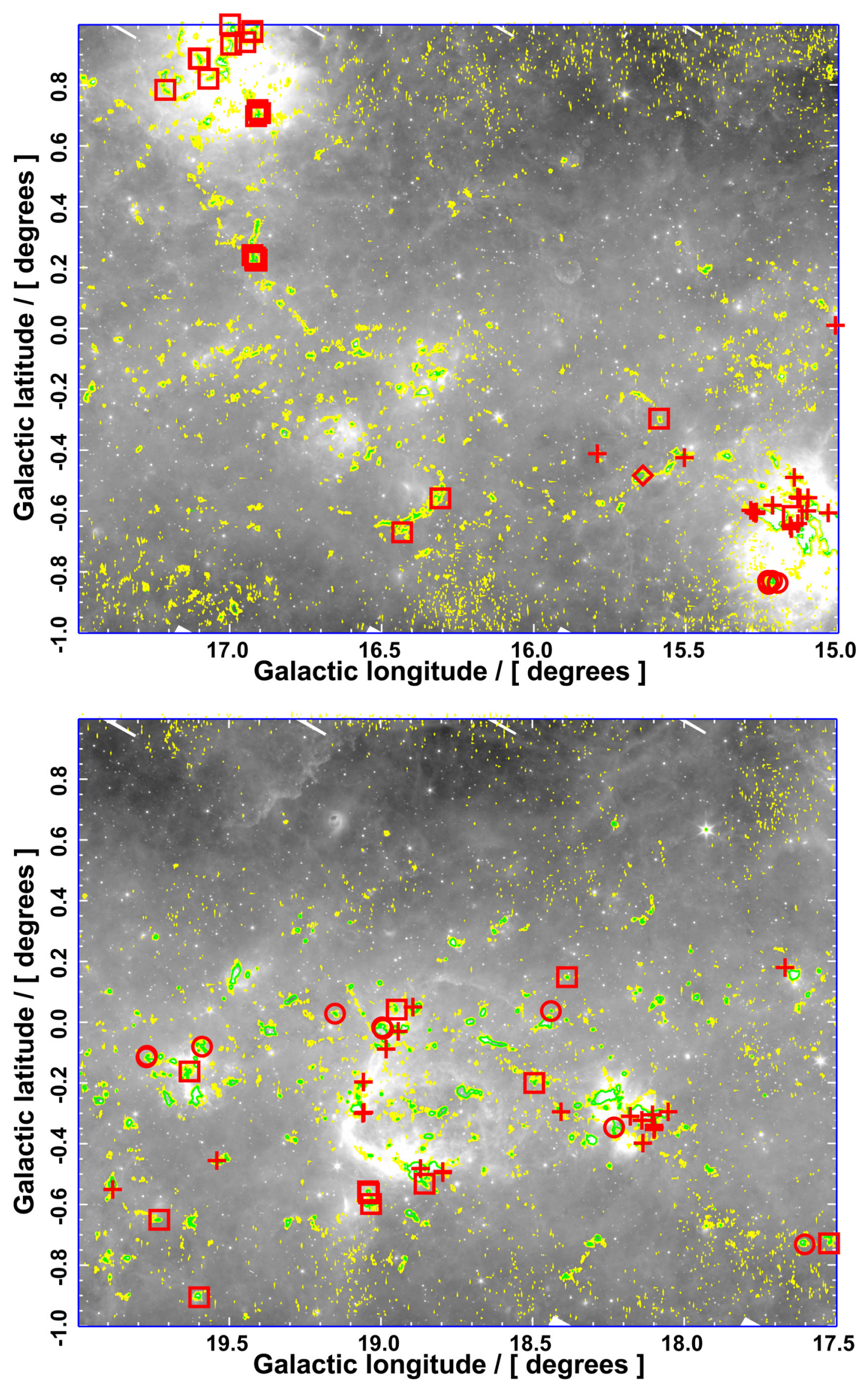

Fig. 12. continued. 


\section{Appendix A: Errors and uncertainties}

As conventional error propagation breaks down when the uncertainties become larger than a few percent, one can only point out the individual sources of errors and estimate the final uncertainties.

The distance error mainly stems from uncertainties in the Galactic rotation curve and errors in the gas velocities can be neglected. Error propagation including the velocity uncertainties following Reid et al. (2009) suggests uncertainties of smaller than $0.1 \mathrm{kpc}$. However, owing to intrinsic errors and deviations from the global Galactic rotation we estimate the distance to be uncertain to within $0.5 \mathrm{kpc}$. This leads to a contribution to the final mass uncertainties ranging from $10 \%$ to $50 \%$, that depends on the absolute distance. In addition, individual objects close to the Galactic center that have non-circular orbits may be placed at random distances and contaminate the sample.
In the literature, temperatures of starless cores range from $10 \mathrm{~K}$ to $20 \mathrm{~K}$ with the bulk at $15 \mathrm{~K}$ (Sridharan et al. 2005; Pillai et al. 2006; Vasyunina et al. 2011; Peretto et al. 2010). The temperature estimate here is based on direct observations of 15 out of 210 starless clumps and is in good agreement with earlier studies. A temperature uncertainty of $\pm 5 \mathrm{~K}$ at $15 \mathrm{~K}$ may introduce mass uncertainties of about a factor of two.

The dust properties and the gas-to-dust ratio are very uncertain as well and might contribute another factor of two to the errors. The flux uncertainties are dominated by the calibration uncertainties, which are $\sim 15 \%$ (Schuller et al. 2009).

When calculating the column density as well as the masses, the predominant uncertainties are those of the dust properties and temperatures. For the mass, the uncertainty in the distance is equally important. Altogether, the total uncertainties in the mass may be as large as a factor of five. 
Appendix B: Omitted regions.

Table B.1. Regions where MIPSGAL $24 \mu \mathrm{m}$ images are to saturated to do a classification.

\begin{tabular}{|c|c|c|c|c|c|c|c|}
\hline Global identifier & Gal lon & Gal lat & $\mathrm{Ra}$ & Dec & Peak flux & $\begin{array}{l}\text { Total } \\
\text { flux } \\
\text { [Jy] }\end{array}$ & Radius \\
\hline 8 & 10.3225 & -0.1608 & 272.2570 & -20.0861 & 4.40 & 45.36 & 50. \\
\hline 45 & 10.1325 & -0.3775 & 272.3610 & -20.3573 & 1.30 & 6.38 & 28. \\
\hline 59 & 10.1392 & -0.3658 & 272.3535 & -20.3458 & 1.06 & 5.59 & 26. \\
\hline 65 & 10.1458 & -0.3158 & 272.3103 & -20.3157 & 1.00 & 7.15 & 31. \\
\hline 70 & 10.1808 & -0.3692 & 272.3781 & -20.3109 & 0.94 & 6.48 & 29. \\
\hline 79 & 10.1575 & -0.3775 & 272.3739 & -20.3354 & 0.88 & 3.34 & 23. \\
\hline 96 & 10.1442 & -0.3558 & 272.3468 & -20.3365 & 0.78 & 2.34 & 19. \\
\hline 465 & 14.9838 & -0.6956 & 275.0954 & -16.2465 & 6.60 & 166.25 & 74. \\
\hline 467 & 15.0121 & -0.7056 & 275.1185 & -16.2262 & 5.24 & 118.69 & 64. \\
\hline 502 & 14.9755 & -0.7139 & 275.1082 & -16.2624 & 1.90 & 27.56 & 49. \\
\hline 514 & 15.0022 & -0.7255 & 275.1320 & -16.2444 & 1.45 & 17.96 & 44. \\
\hline 519 & 14.9938 & -0.7305 & 275.1325 & -16.2541 & 1.34 & 12.58 & 36. \\
\hline 554 & 14.9738 & -0.7405 & 275.1318 & -16.2765 & 0.92 & 4.37 & 25. \\
\hline 561 & 14.9755 & -0.7372 & 275.1296 & -16.2734 & 0.91 & 6.55 & 28. \\
\hline 617 & 14.9688 & -0.7389 & 275.1279 & -16.2801 & 0.72 & 2.42 & 20. \\
\hline 656 & 13.9908 & -0.1208 & 274.0768 & -16.8488 & 0.61 & 1.40 & 16. \\
\hline 687 & 12.7975 & -0.2275 & 273.5786 & -17.9485 & 0.56 & 2.69 & 23. \\
\hline 707 & 13.9892 & -0.1358 & 274.0897 & -16.8574 & 0.54 & 1.52 & 18. \\
\hline 786 & 14.9738 & -0.7539 & 275.1441 & -16.2827 & 0.50 & 1.33 & 17. \\
\hline 1114 & 15.0545 & -0.6256 & 275.0659 & -16.1512 & 3.59 & 28.11 & 40. \\
\hline 1116 & 15.0511 & -0.6423 & 275.0795 & -16.1620 & 2.98 & 46.09 & 50. \\
\hline 1119 & 15.0678 & -0.6140 & 275.0617 & -16.1339 & 2.61 & 22.05 & 37. \\
\hline 1122 & 15.0995 & -0.6889 & 275.1461 & -16.1414 & 2.25 & 22.50 & 38. \\
\hline 1125 & 15.0778 & -0.6073 & 275.0605 & -16.1220 & 2.02 & 21.50 & 36. \\
\hline 1126 & 15.1844 & -0.6223 & 275.1266 & -16.0351 & 1.96 & 37.36 & 58. \\
\hline 1131 & 15.1311 & -0.6706 & 275.1448 & -16.1048 & 1.74 & 21.13 & 46. \\
\hline 1137 & 15.1028 & -0.6573 & 275.1187 & -16.1235 & 1.40 & 31.14 & 56. \\
\hline 1139 & 15.0028 & -0.7206 & 275.1277 & -16.2415 & 1.36 & 9.88 & 30. \\
\hline 1140 & 14.9929 & -0.7322 & 275.1335 & -16.2558 & 1.36 & 11.98 & 35. \\
\hline 1141 & 15.1061 & -0.6939 & 275.1539 & -16.1378 & 1.36 & 12.79 & 36. \\
\hline 1152 & 15.1111 & -0.7122 & 275.1732 & -16.1421 & 1.16 & 11.03 & 36. \\
\hline 1153 & 15.1161 & -0.6340 & 275.1038 & -16.1008 & 1.16 & 9.14 & 32. \\
\hline 1157 & 15.0961 & -0.7106 & 275.1643 & -16.1545 & 1.10 & 5.29 & 26. \\
\hline 1168 & 15.0944 & -0.7139 & 275.1666 & -16.1576 & 0.95 & 3.61 & 22. \\
\hline 1174 & 15.0944 & -0.6073 & 275.0687 & -16.1073 & 0.92 & 4.57 & 23. \\
\hline 1180 & 15.1078 & -0.6273 & 275.0936 & -16.1050 & 0.91 & 5.14 & 25. \\
\hline 1181 & 15.1061 & -0.5923 & 275.0606 & -16.0900 & 0.90 & 5.60 & 29. \\
\hline 1187 & 15.0944 & -0.7322 & 275.1834 & -16.1662 & 0.85 & 4.65 & 27. \\
\hline 1193 & 15.0412 & -0.6189 & 275.0532 & -16.1598 & 0.81 & 5.39 & 28. \\
\hline 1202 & 15.1278 & -0.6923 & 275.1630 & -16.1180 & 0.76 & 3.21 & 23. \\
\hline 1207 & 14.9845 & -0.7455 & 275.1417 & -16.2694 & 0.74 & 2.95 & 22. \\
\hline 1214 & 15.0995 & -0.7322 & 275.1859 & -16.1618 & 0.73 & 2.98 & 22. \\
\hline 1215 & 15.0778 & -0.6823 & 275.1293 & -16.1573 & 0.72 & 4.79 & 27. \\
\hline 1223 & 15.1028 & -0.6223 & 275.0865 & -16.1070 & 0.71 & 3.04 & 22. \\
\hline 1224 & 14.9845 & -0.6589 & 275.0621 & -16.2286 & 0.71 & 4.20 & 26. \\
\hline 1231 & 15.1278 & -0.7023 & 275.1722 & -16.1227 & 0.68 & 1.34 & 16. \\
\hline 1233 & 15.0861 & -0.6273 & 275.0829 & -16.1241 & 0.68 & 2.48 & 21. \\
\hline 1285 & 15.1228 & -0.7106 & 275.1774 & -16.1310 & 0.54 & 1.19 & 15. \\
\hline 1291 & 15.0561 & -0.6989 & 275.1340 & -16.1843 & 0.53 & 1.55 & 18. \\
\hline 1294 & 14.9879 & -0.7555 & 275.1525 & -16.2712 & 0.53 & 0.87 & 14. \\
\hline 1297 & 15.0328 & -0.7372 & 275.1577 & -16.2229 & 0.52 & 1.78 & 19. \\
\hline 1301 & 15.1094 & -0.5823 & 275.0531 & -16.0823 & 0.52 & 1.33 & 17. \\
\hline 1302 & 15.1244 & -0.7023 & 275.1706 & -16.1256 & 0.52 & 1.18 & 15. \\
\hline 1303 & 15.0245 & -0.7389 & 275.1552 & -16.2310 & 0.52 & 0.68 & 12. \\
\hline 1315 & 15.1311 & -0.6856 & 275.1585 & -16.1119 & 0.52 & 2.16 & 21. \\
\hline 1324 & 15.1011 & -0.6323 & 275.0949 & -16.1132 & 0.51 & 1.64 & 18. \\
\hline 1327 & 15.0628 & -0.6023 & 275.0486 & -16.1328 & 0.51 & 1.82 & 19. \\
\hline 1331 & 15.0278 & -0.7339 & 275.1522 & -16.2257 & 0.51 & 1.68 & 18. \\
\hline 1737 & 19.0742 & -0.2742 & 276.6897 & -12.4356 & 0.52 & 2.73 & 23. \\
\hline
\end{tabular}

Notes. Regions listed below were omitted during the classification. Columns are identifier, galactic longitude, galactic latitude, right ascension, declination, peak flux, integrated flux and radius as calculated by CLUMPFIND. 
J. Tackenberg et al.: Starless clumps in ATLASGAL

\section{Appendix C: Stamps of starless regions}

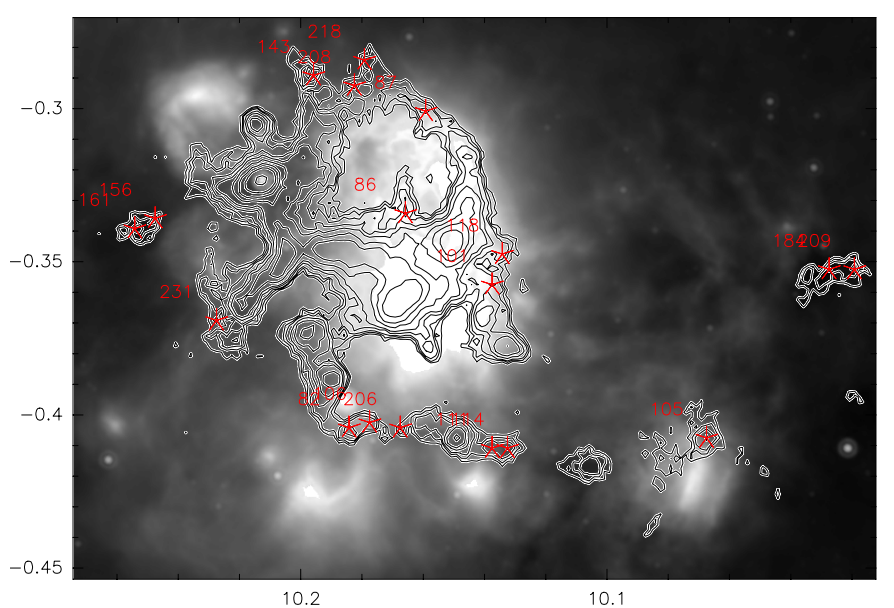

Fig. C.1. MIPSGAL $24 \mu \mathrm{m}$ image with ATLASGAL contours on top. Starless clumps are marked with a red asterisk. The numbers correspond to the global identifier given in Table 3.

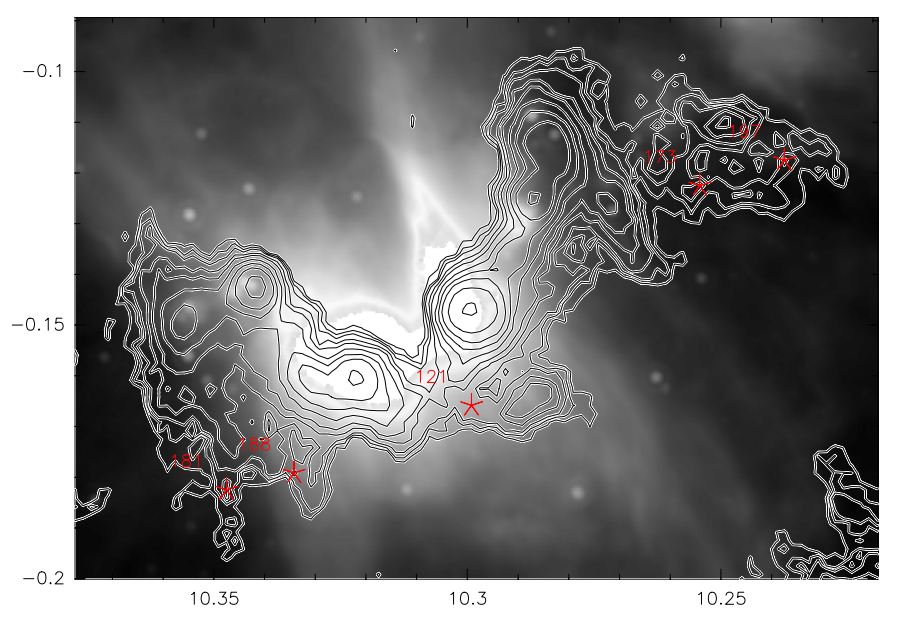

Fig. C.1. continued.

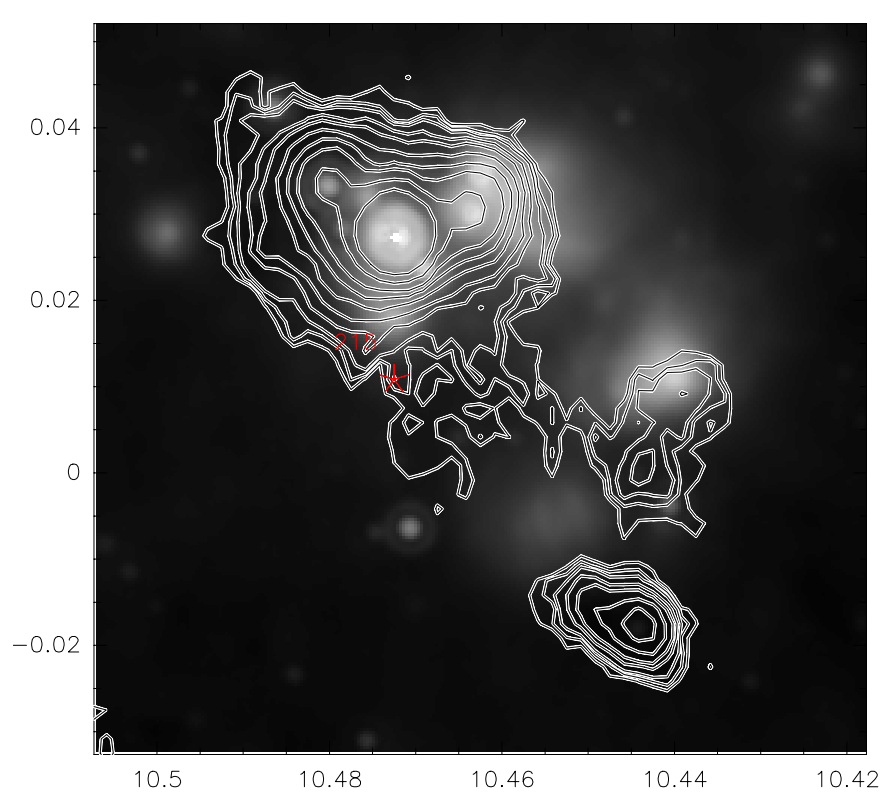

Fig. C.1. continued.

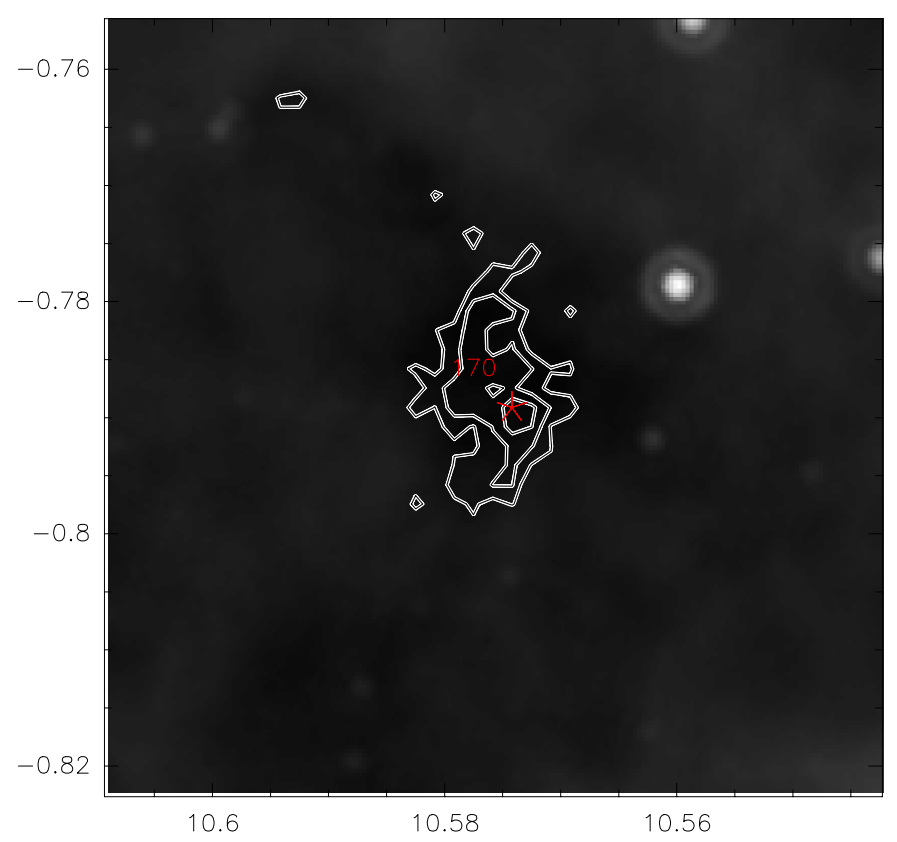

Fig. C.1. continued.

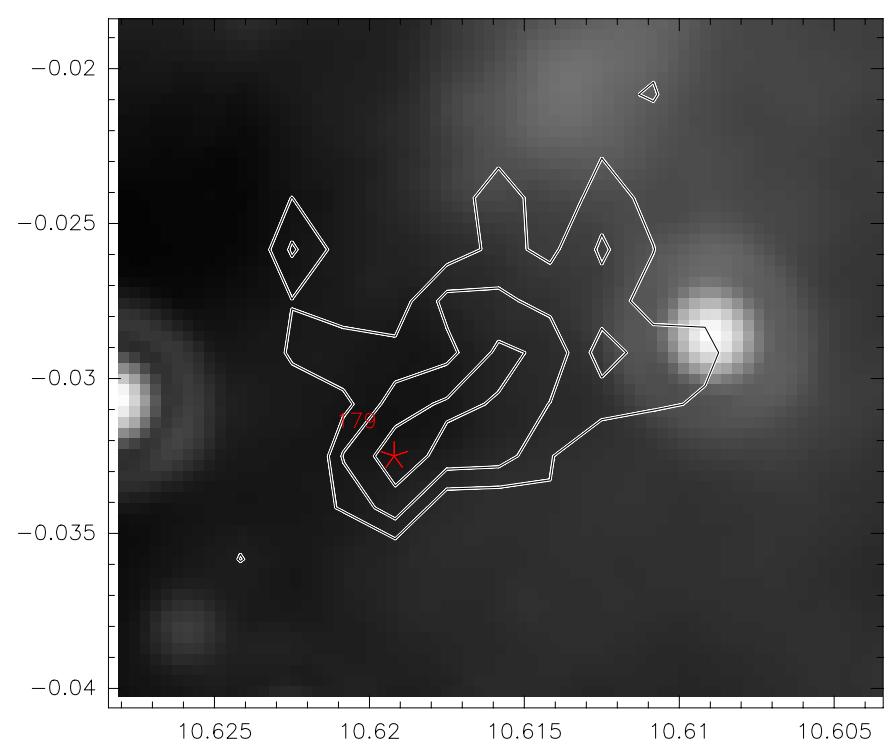

Fig. C.1. continued. 
A\&A 540, A113 (2012)

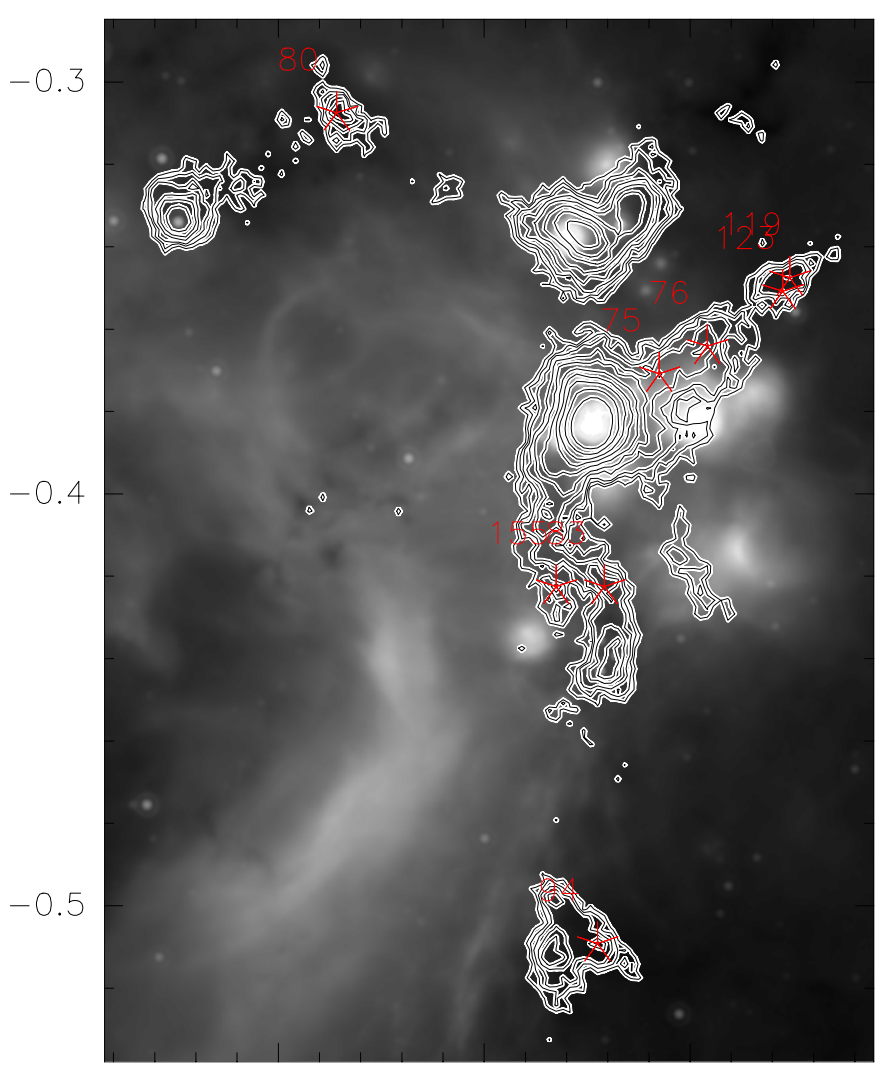

10.7

10.65

10.6

Fig. C.1. continued.

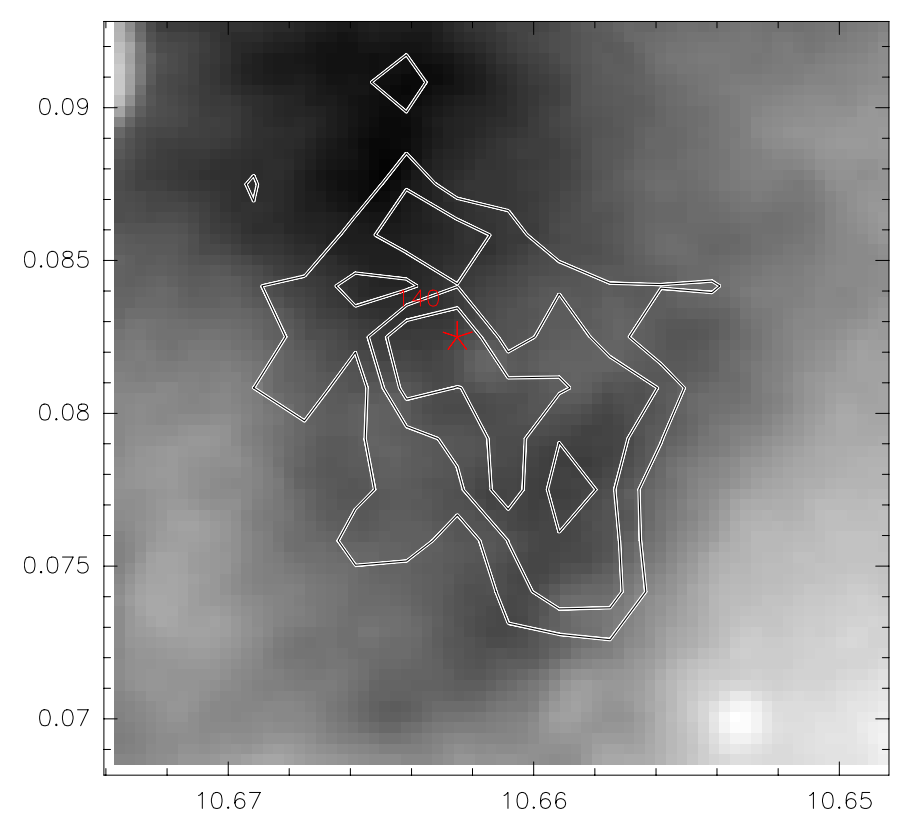

Fig. C.1. continued.

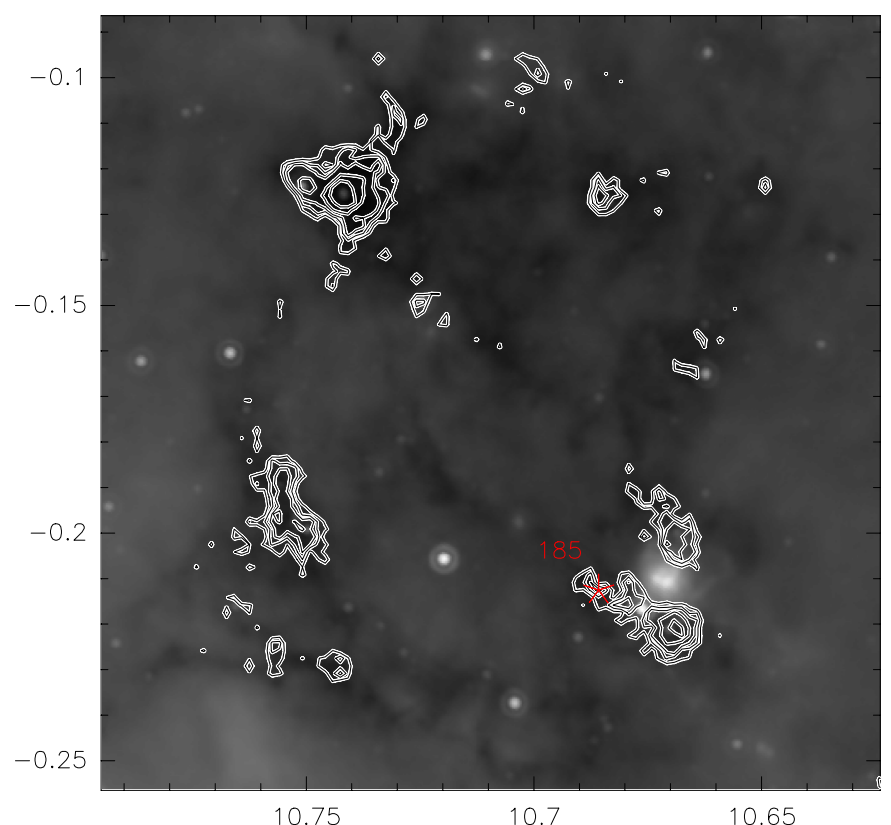

Fig. C.1. continued.

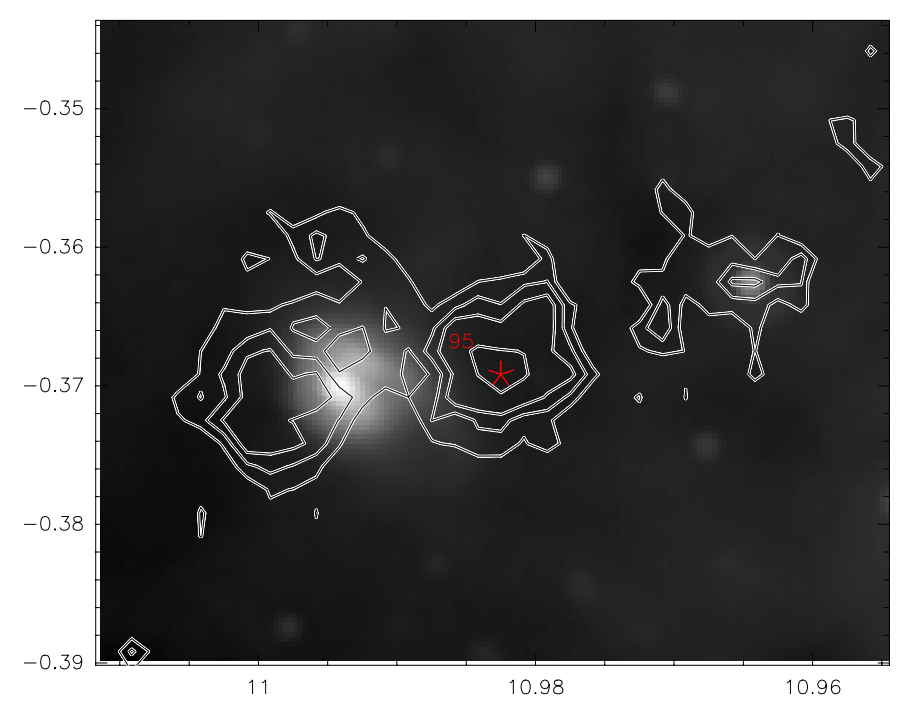

Fig. C.1. continued.

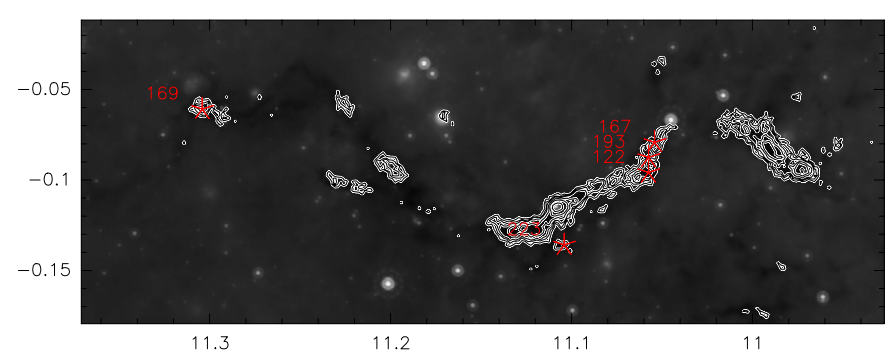

Fig. C.1. continued. 
J. Tackenberg et al.: Starless clumps in ATLASGAL
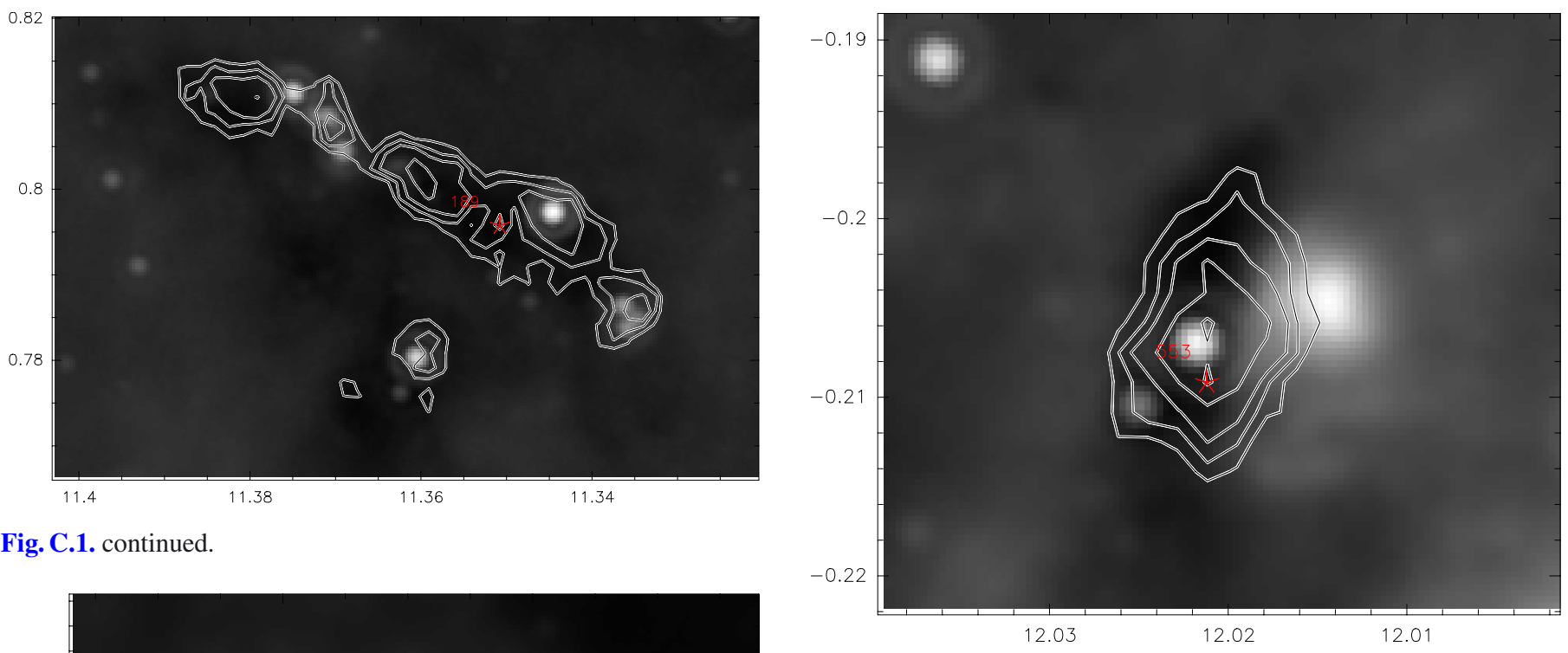

Fig. C.1. continued.

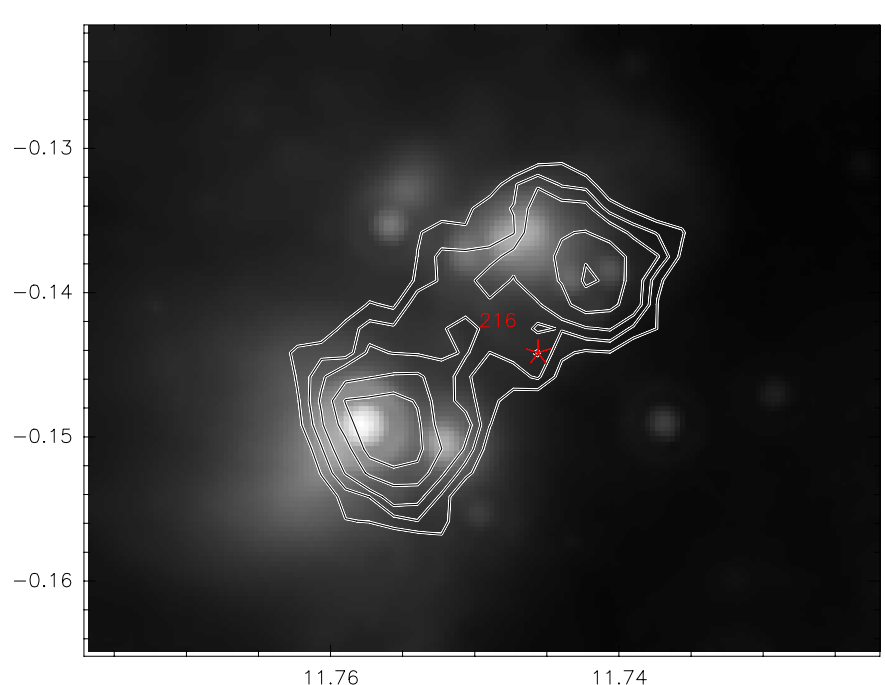

Fig. C.1. continued.

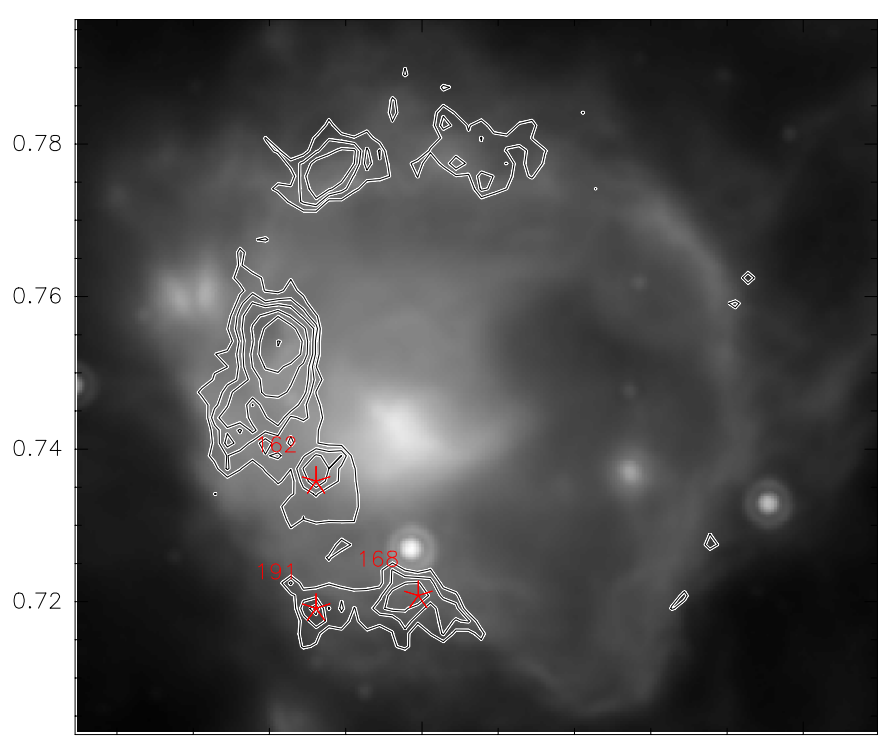

Fig. C.1. continued.

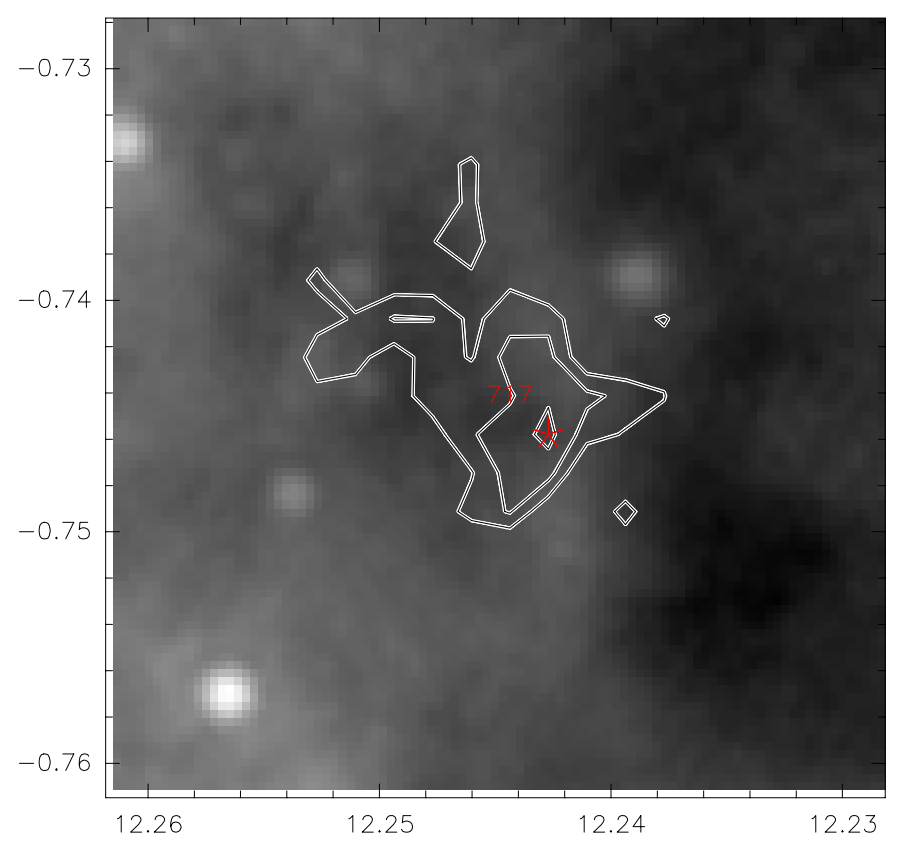

Fig. C.1. continued.

Fig. C.1. continued. 
A\&A 540, A113 (2012)

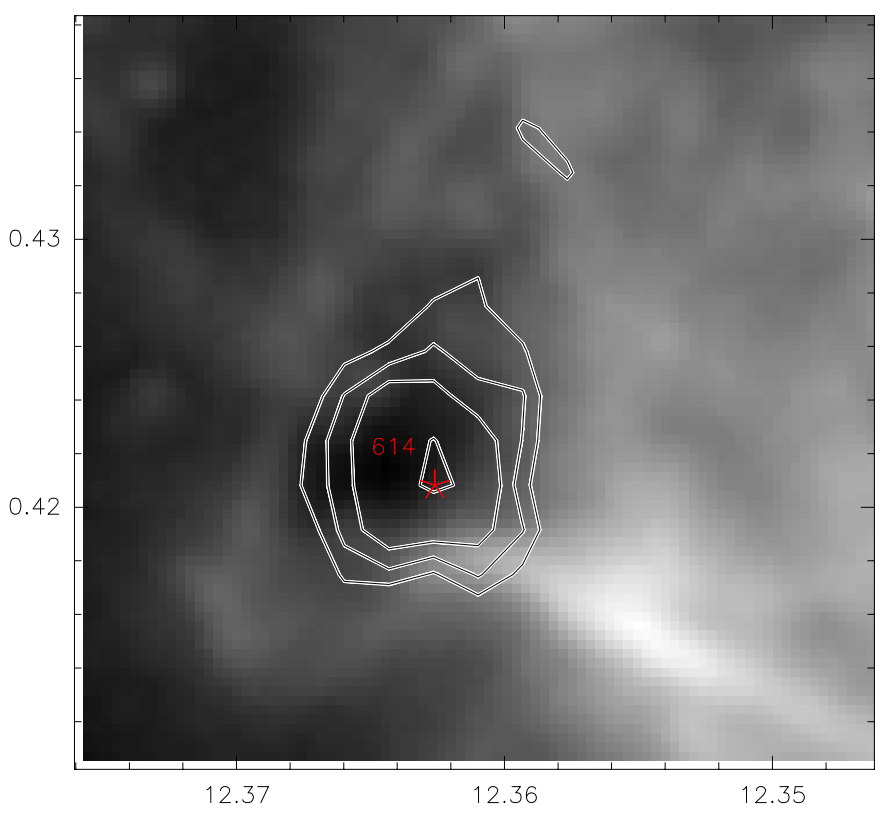

Fig. C.1. continued.

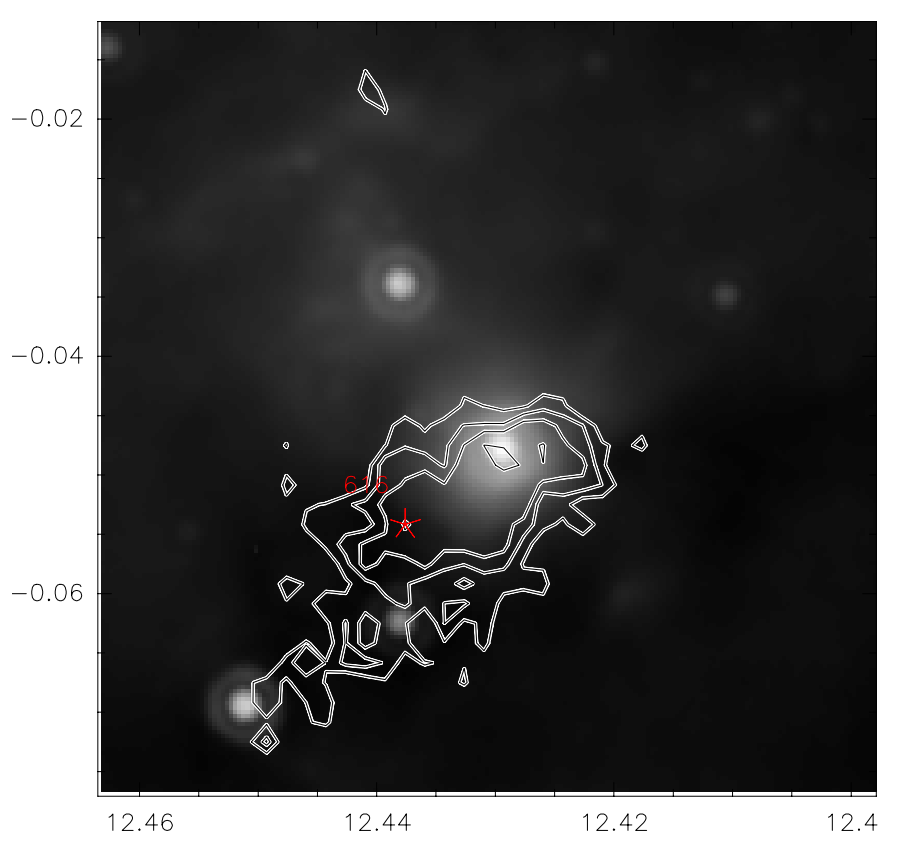

Fig. C.1. continued.

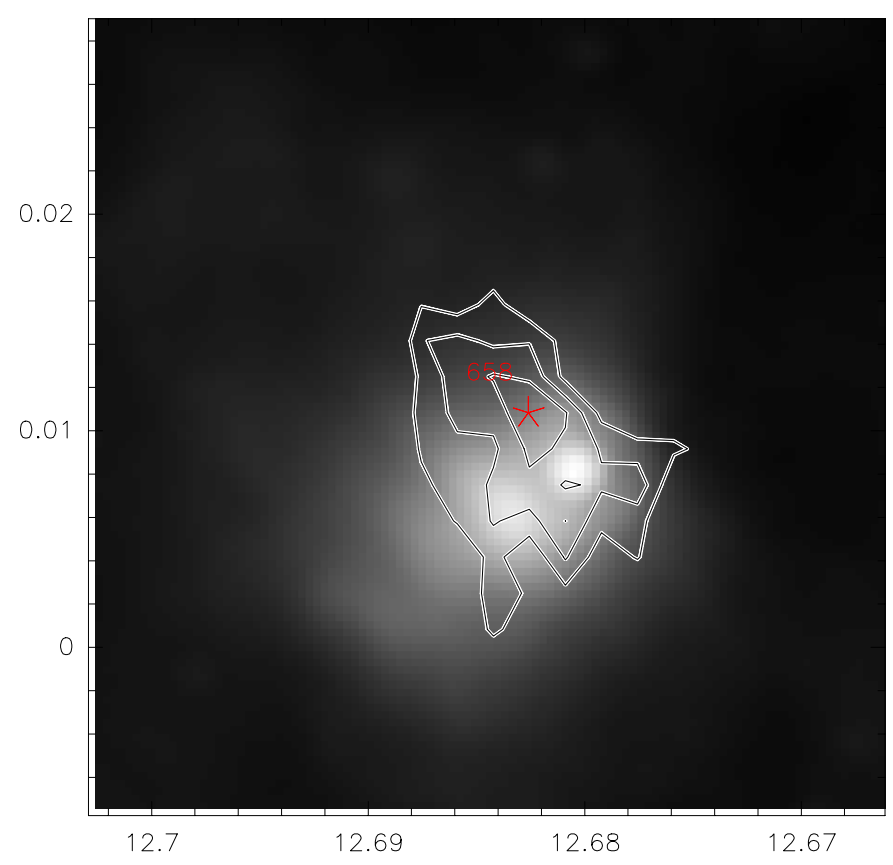

Fig. C.1. continued.

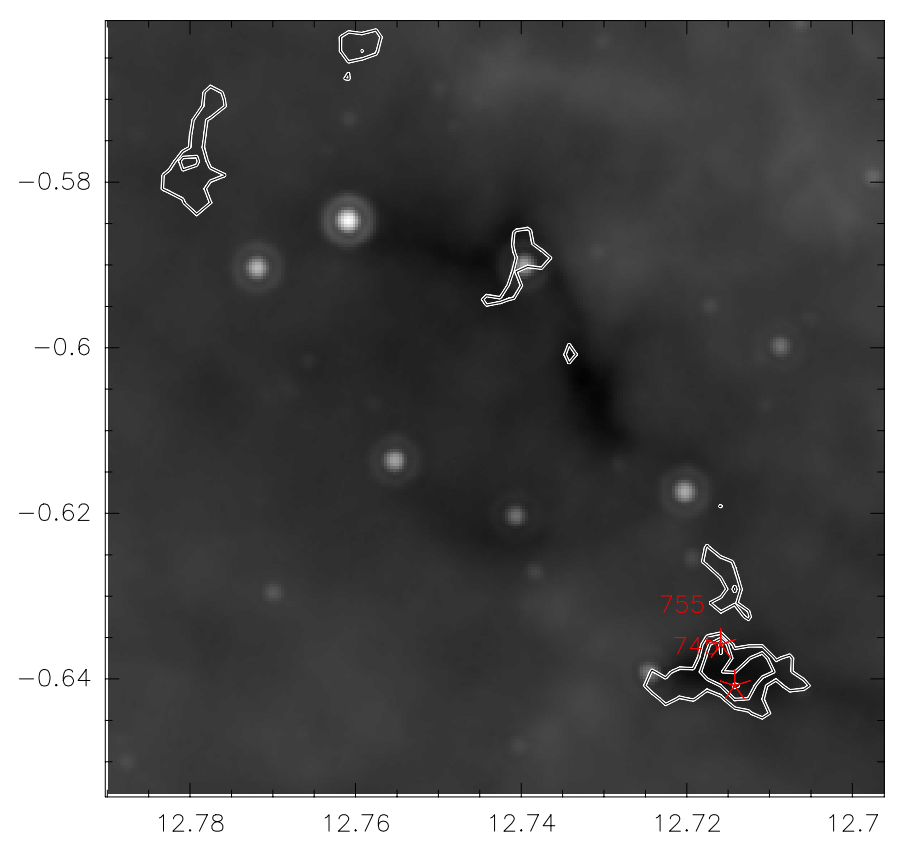

Fig. C.1. continued. 
J. Tackenberg et al.: Starless clumps in ATLASGAL
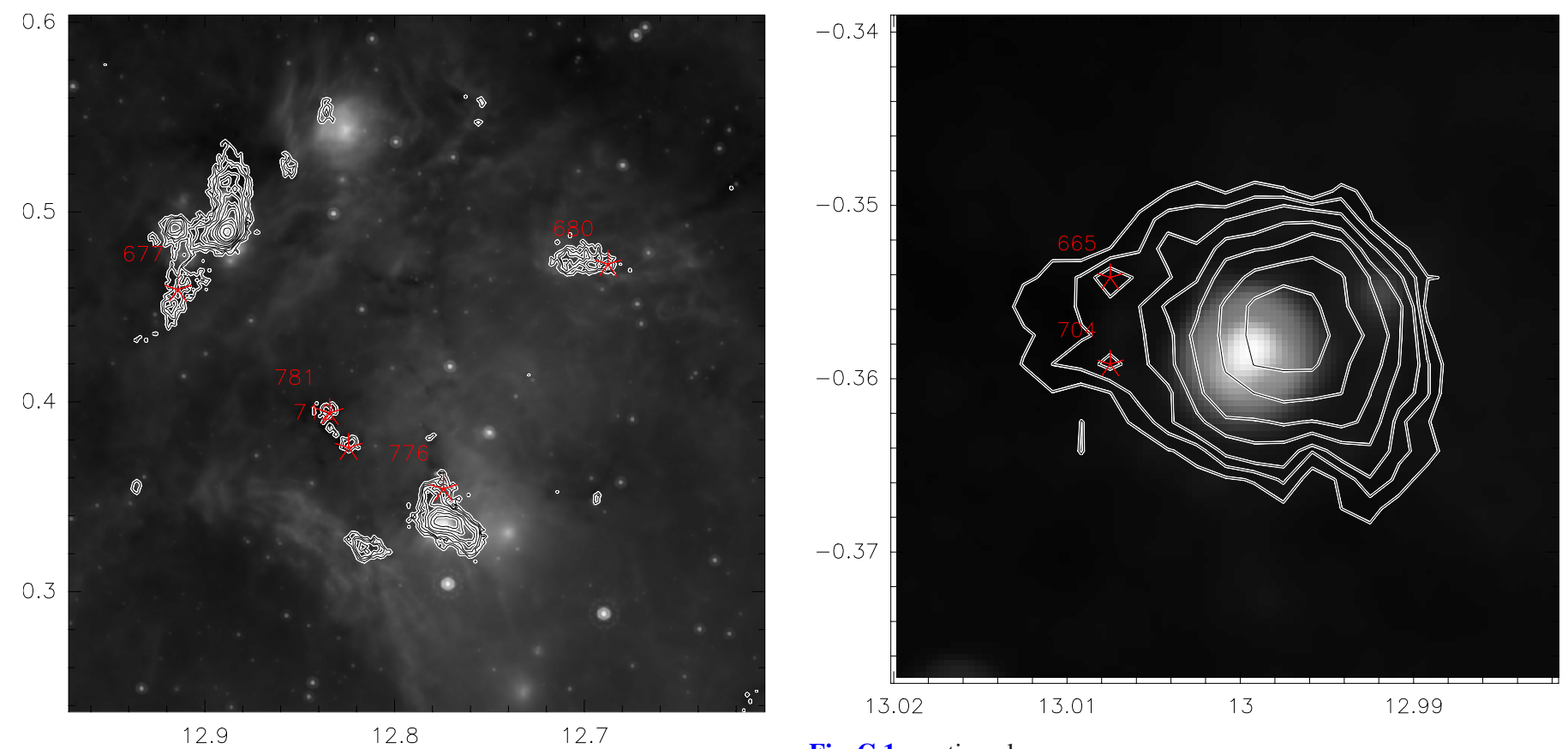

Fig. C.1. continued.

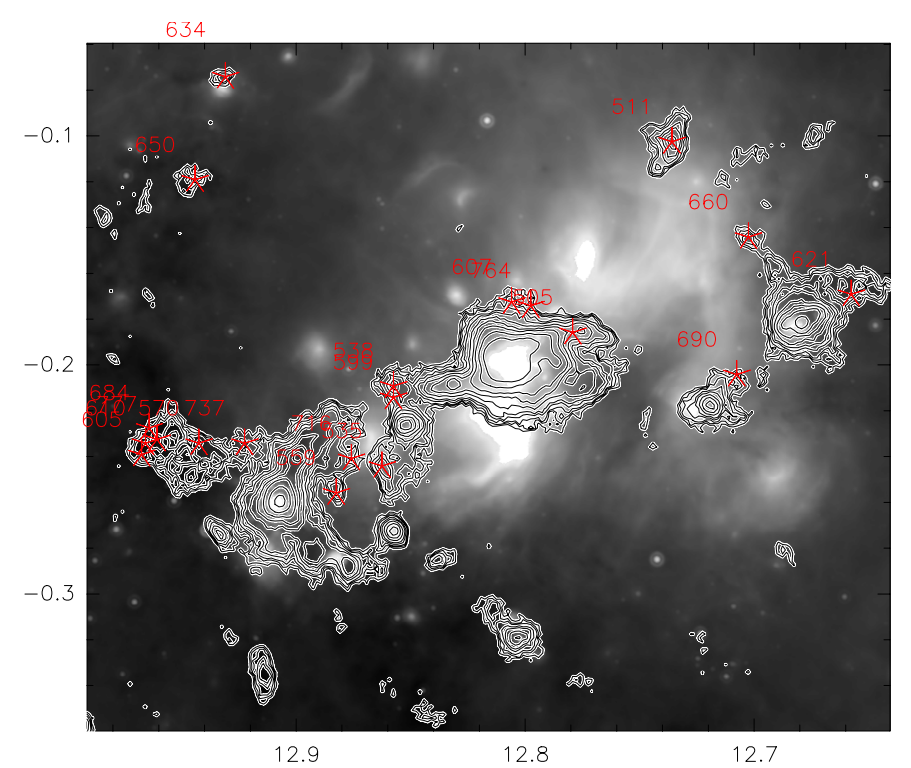

Fig. C.1. continued.

Fig. C.1. continued.

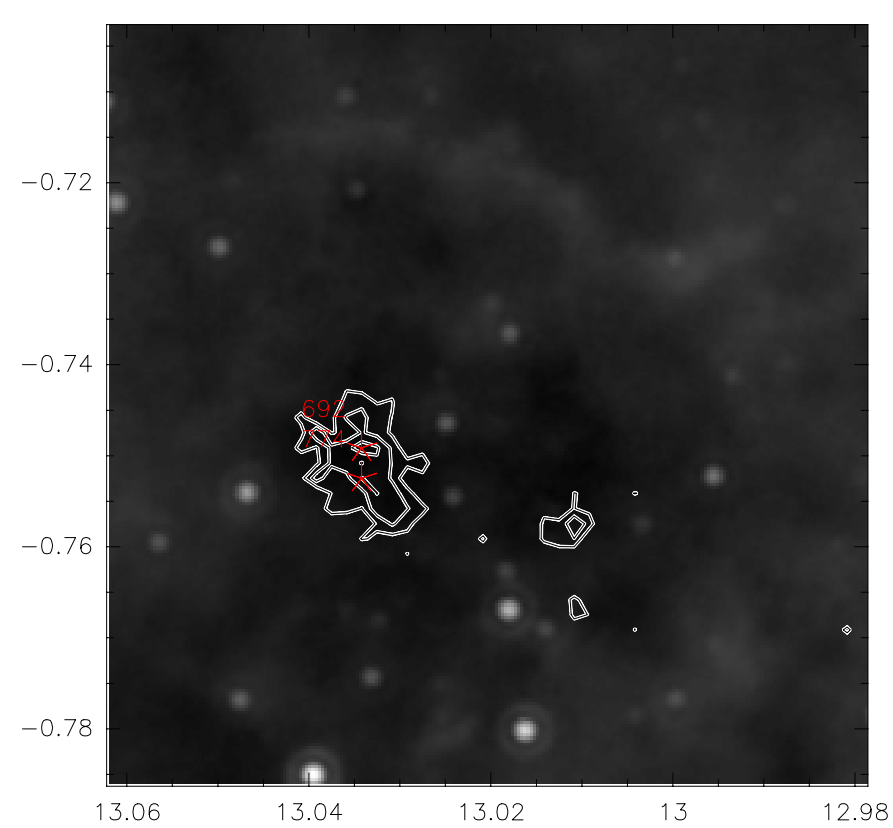

Fig. C.1. continued. 
A\&A 540, A113 (2012)
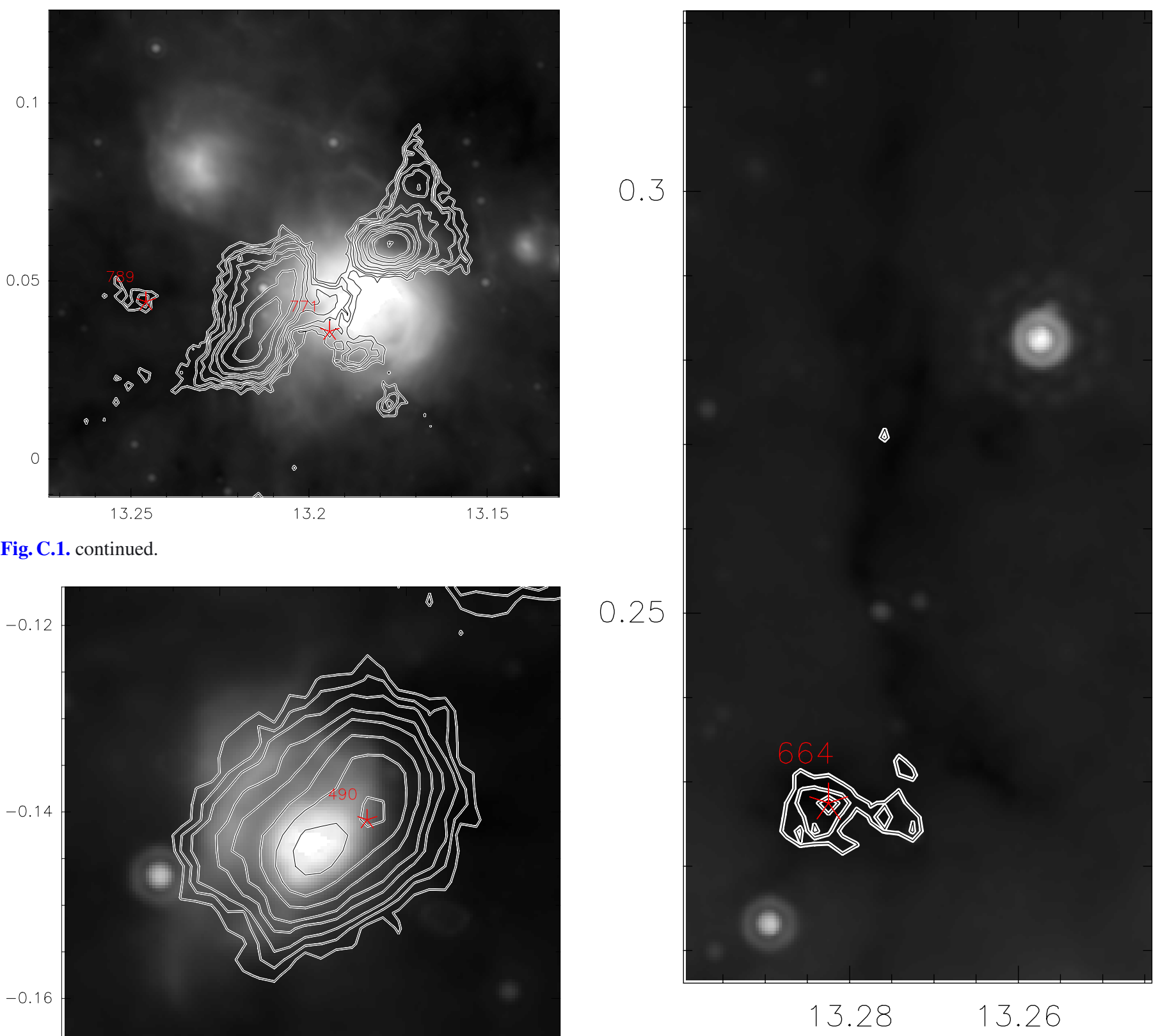

Fig. C.1. continued.

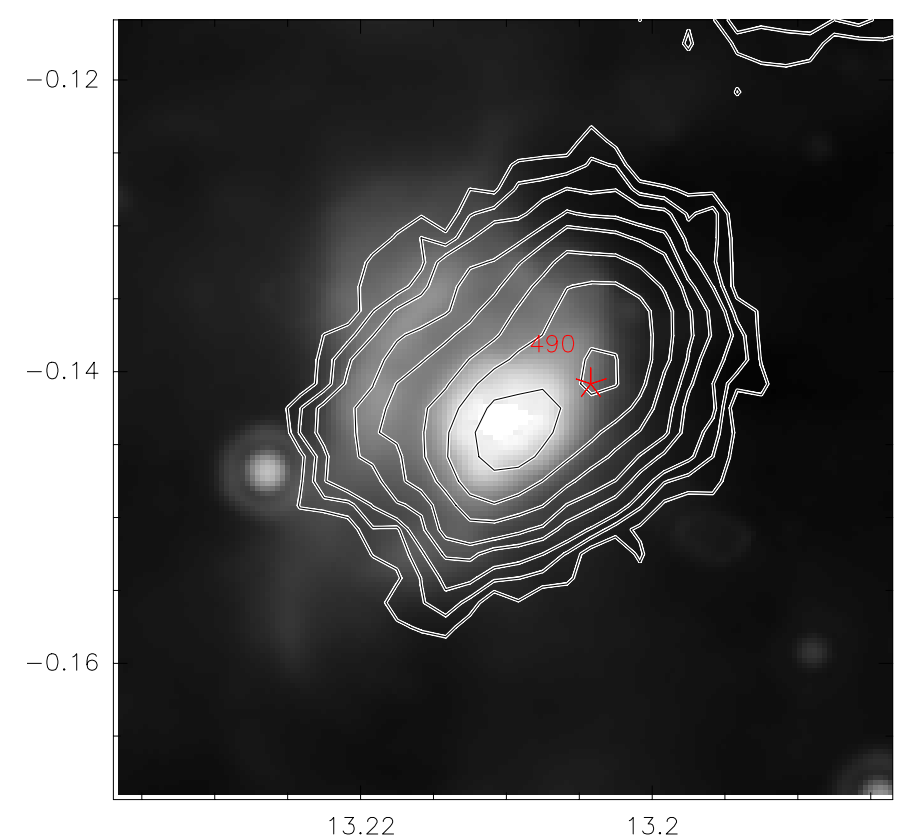

Fig. C.1. continued.

Fig. C.1. continued.

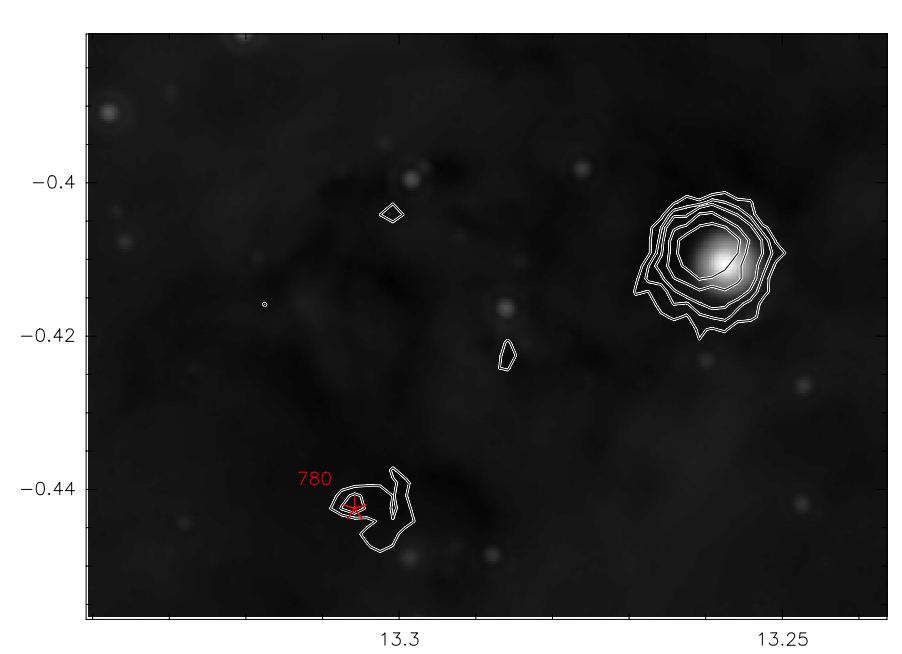

Fig. C.1. continued. 
J. Tackenberg et al.: Starless clumps in ATLASGAL
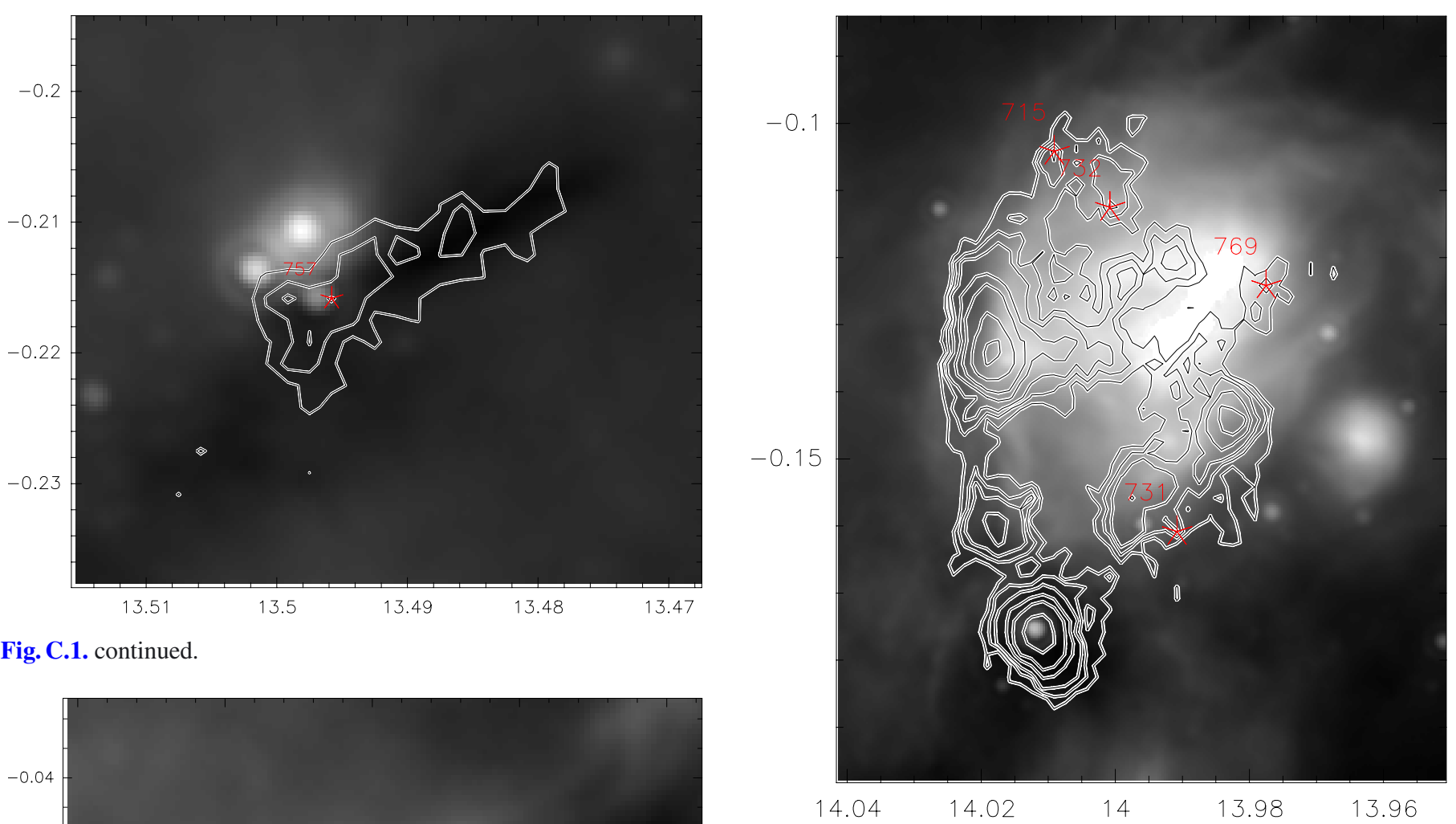

Fig. C.1. continued.

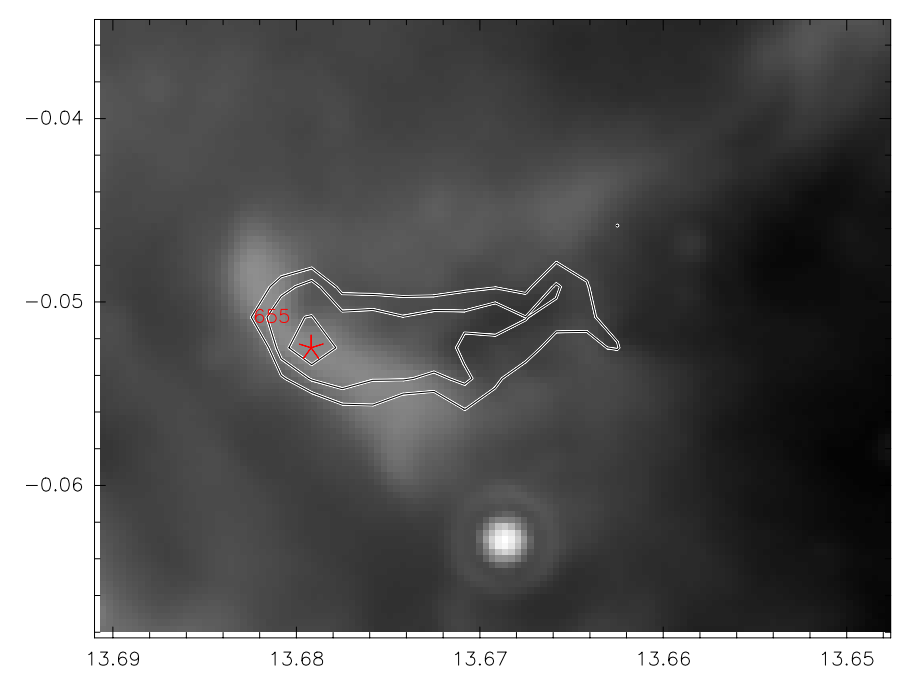

Fig. C.1. continued.

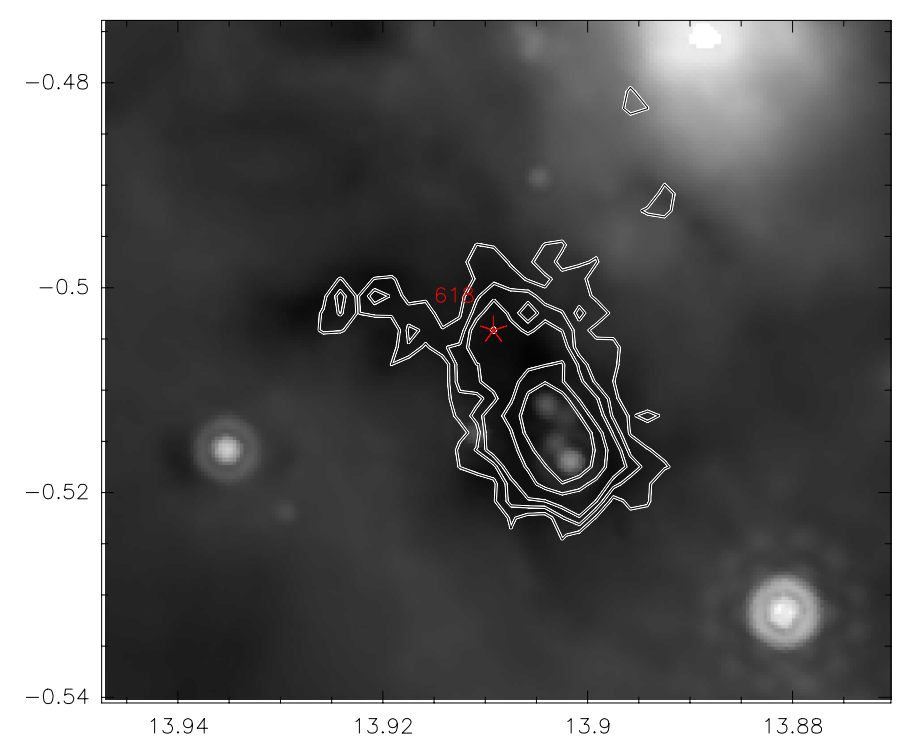

Fig. C.1. continued.

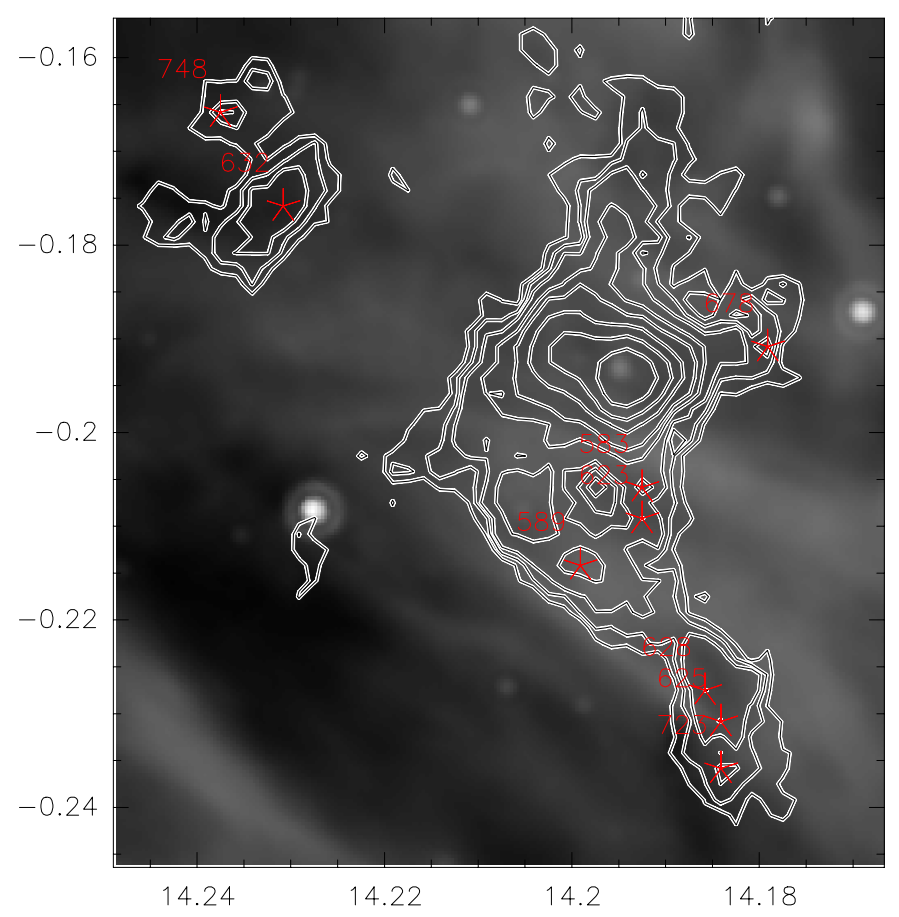

Fig. C.1. continued.

Fig. C.1. continued. 
A\&A 540, A113 (2012)

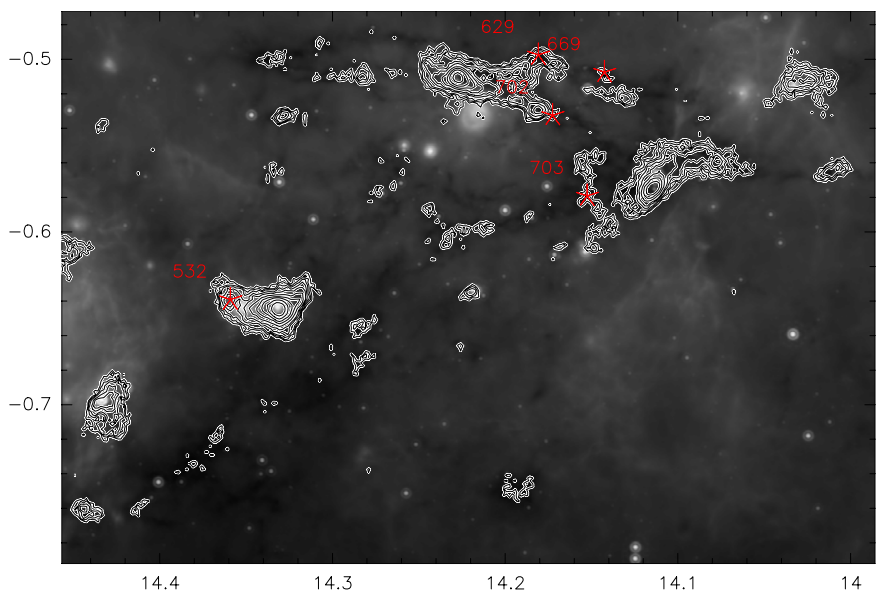

Fig. C.1. continued.

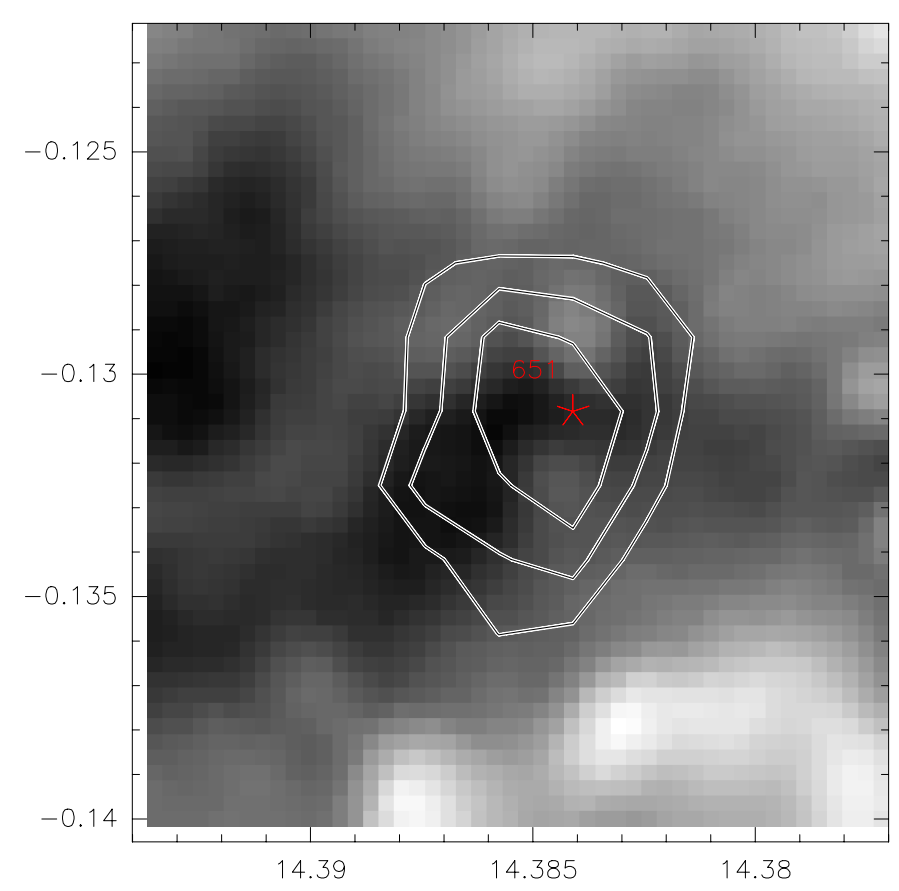

Fig. C.1. continued.

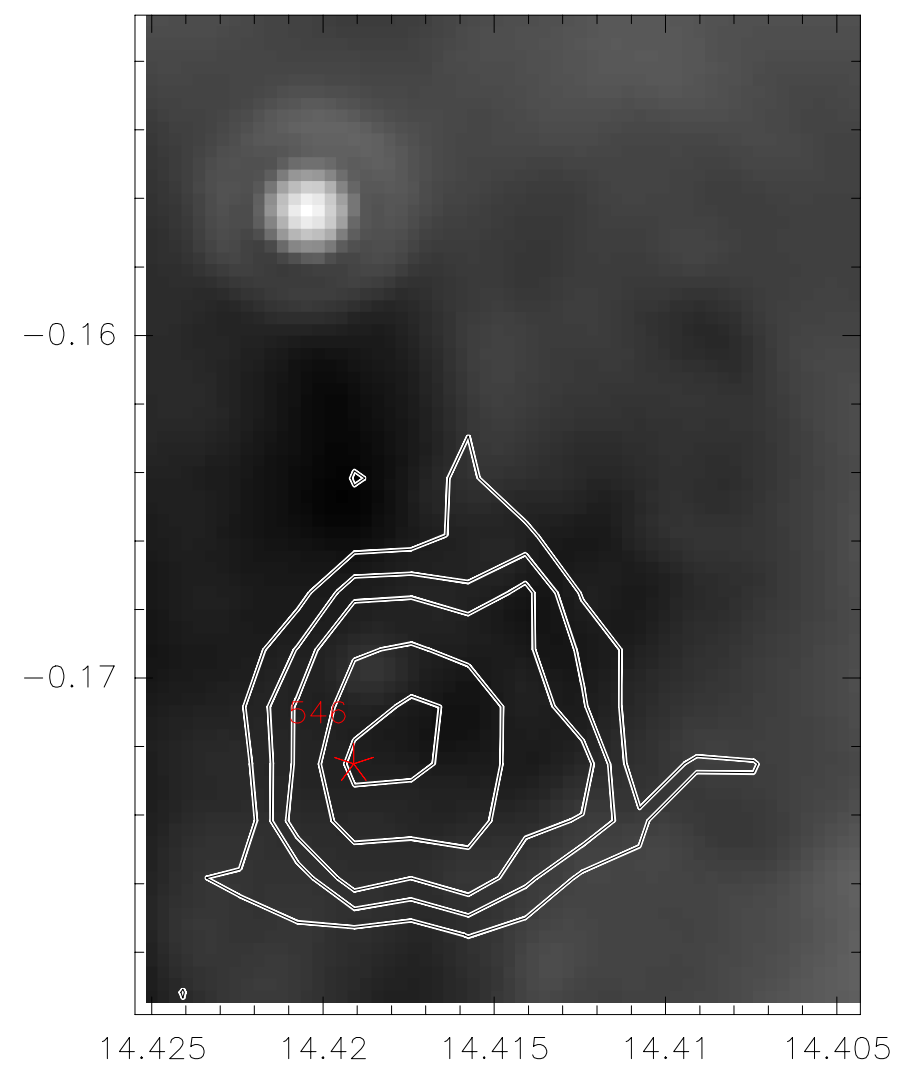

Fig. C.1. continued.

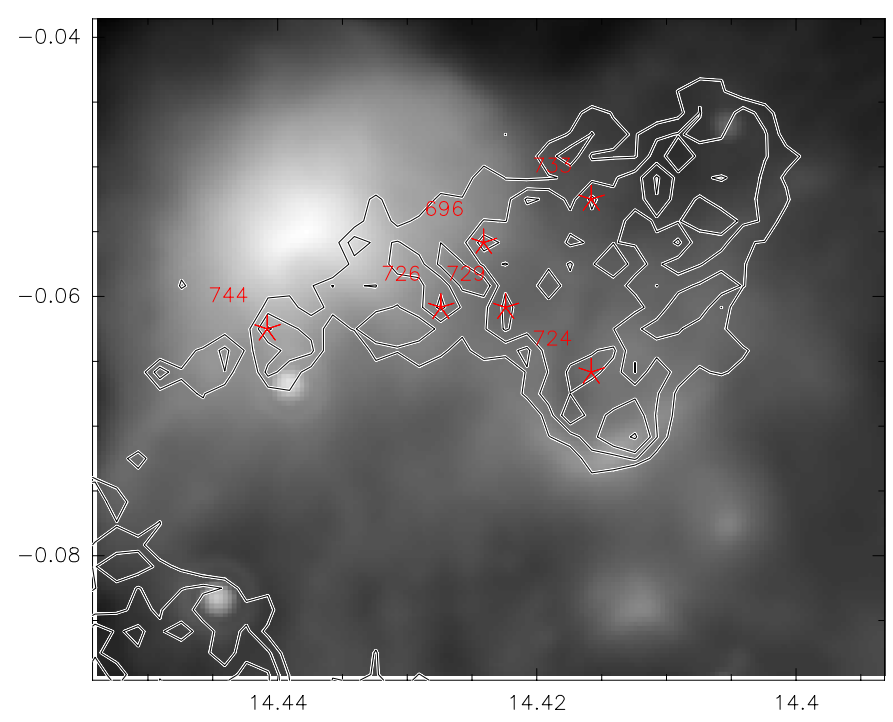

Fig. C.1. continued. 
J. Tackenberg et al.: Starless clumps in ATLASGAL

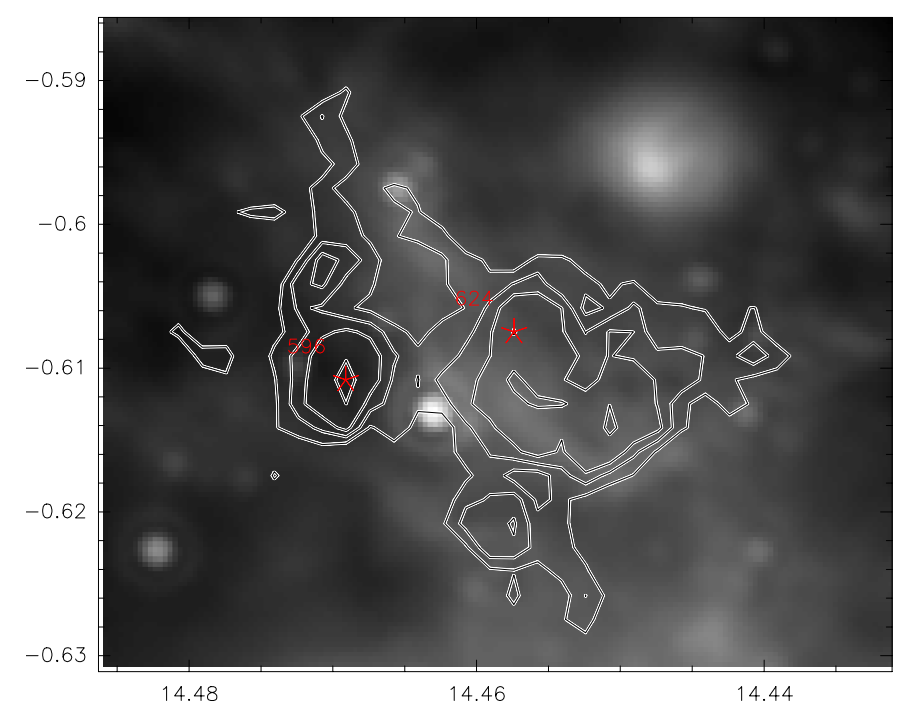

Fig. C.1. continued.

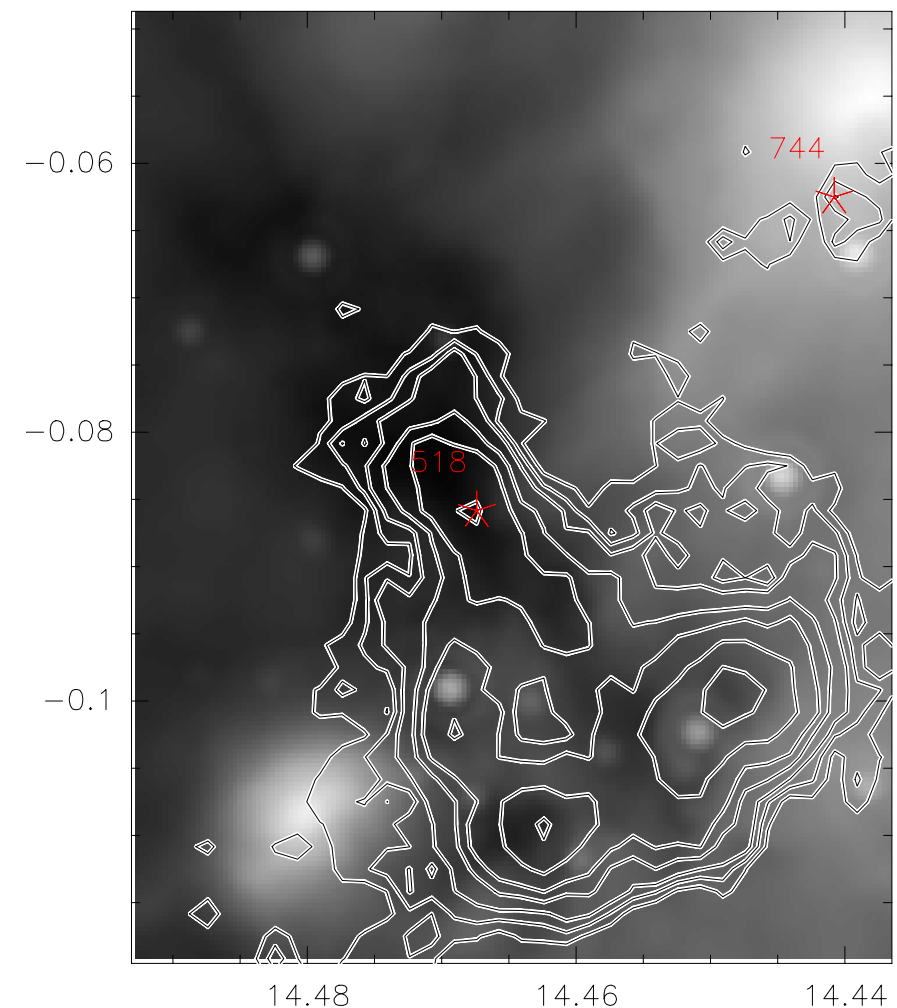

Fig. C.1. continued.

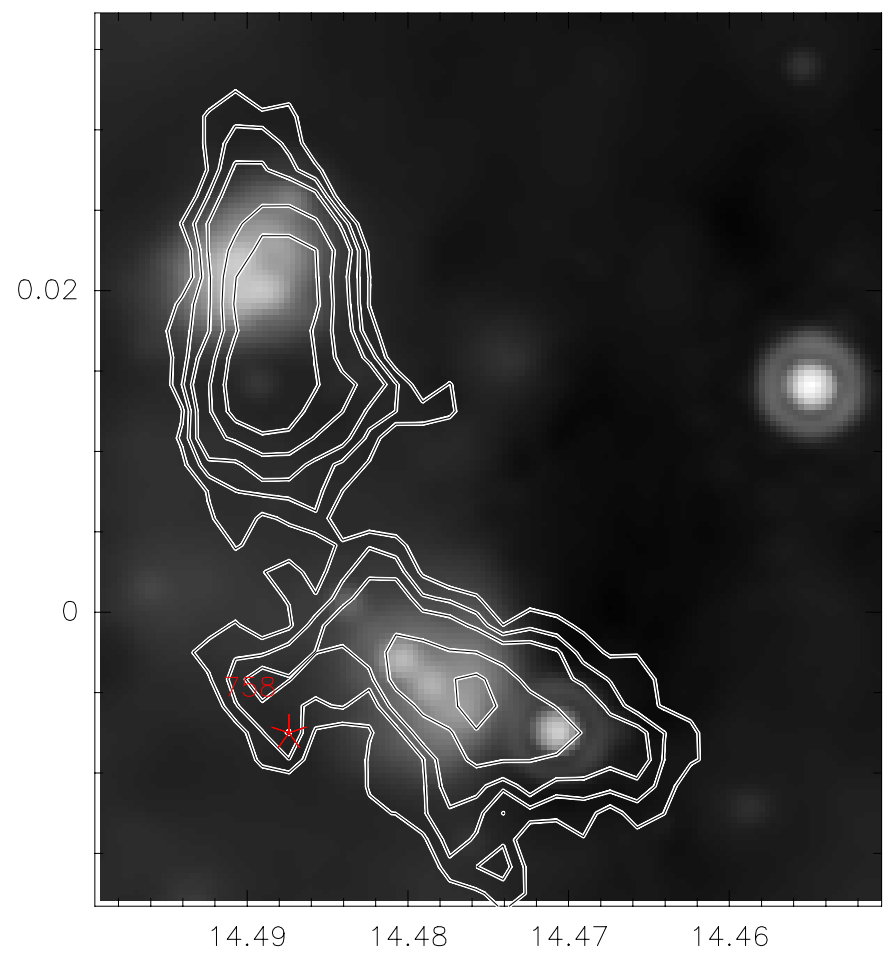

Fig. C.1. continued.

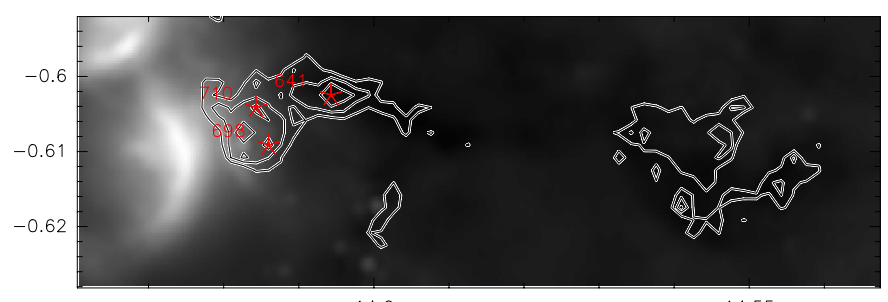

Fig. C.1. continued.

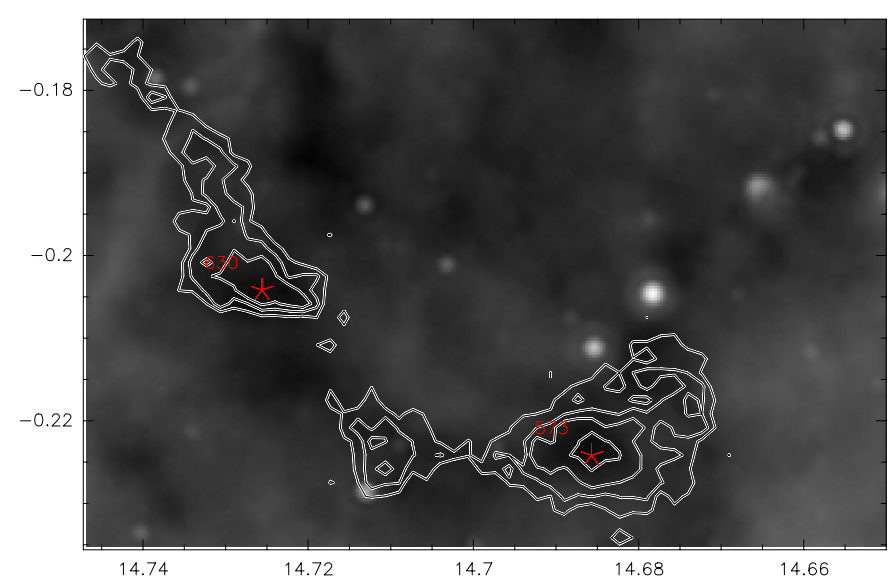

Fig. C.1. continued. 
A\&A 540, A113 (2012)

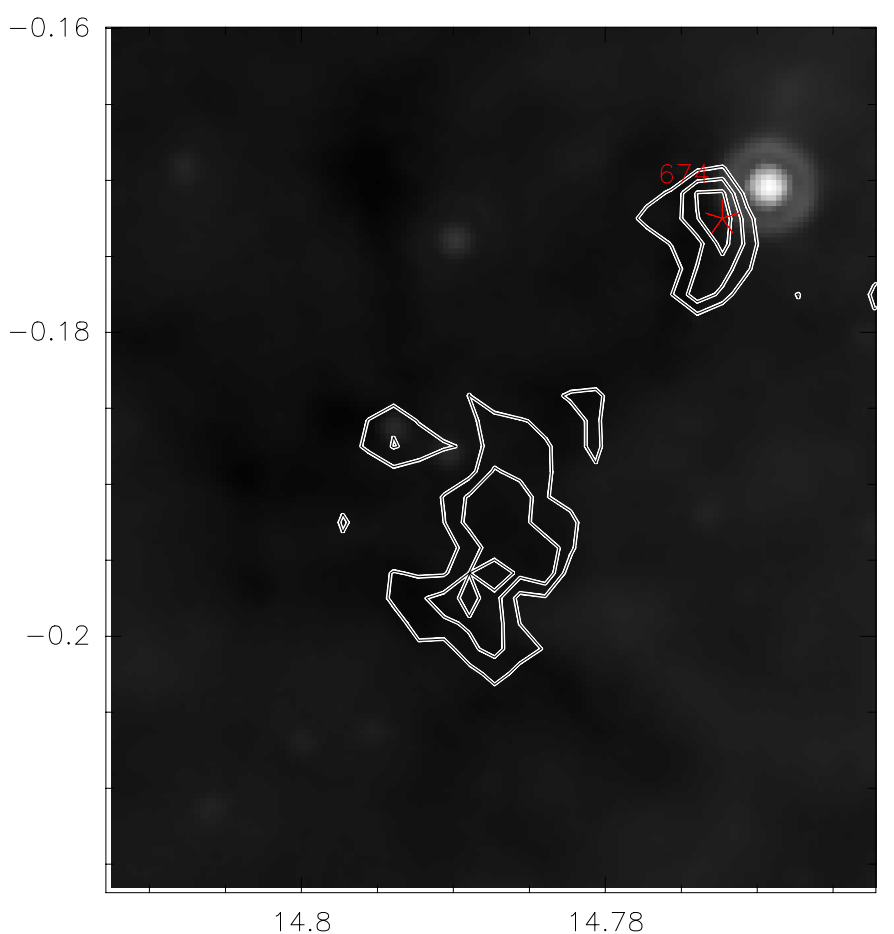

Fig. C.1. continued.

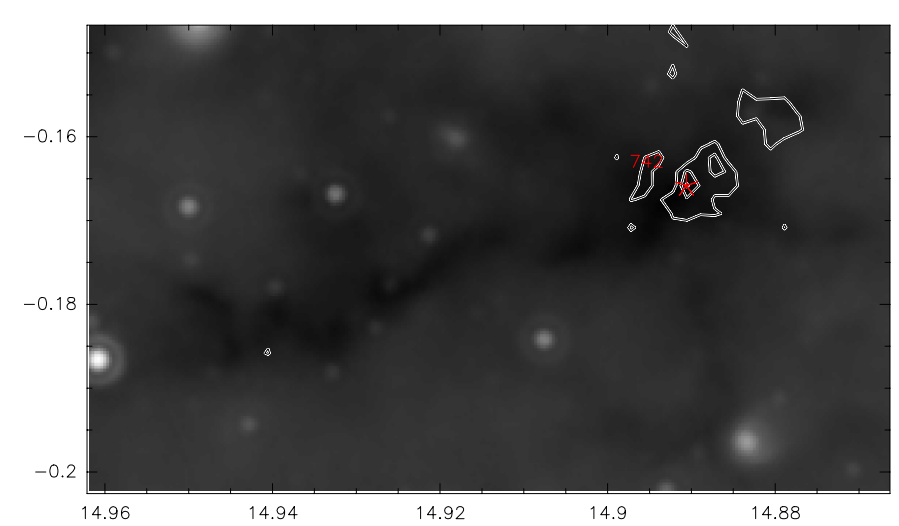

Fig. C.1. continued.

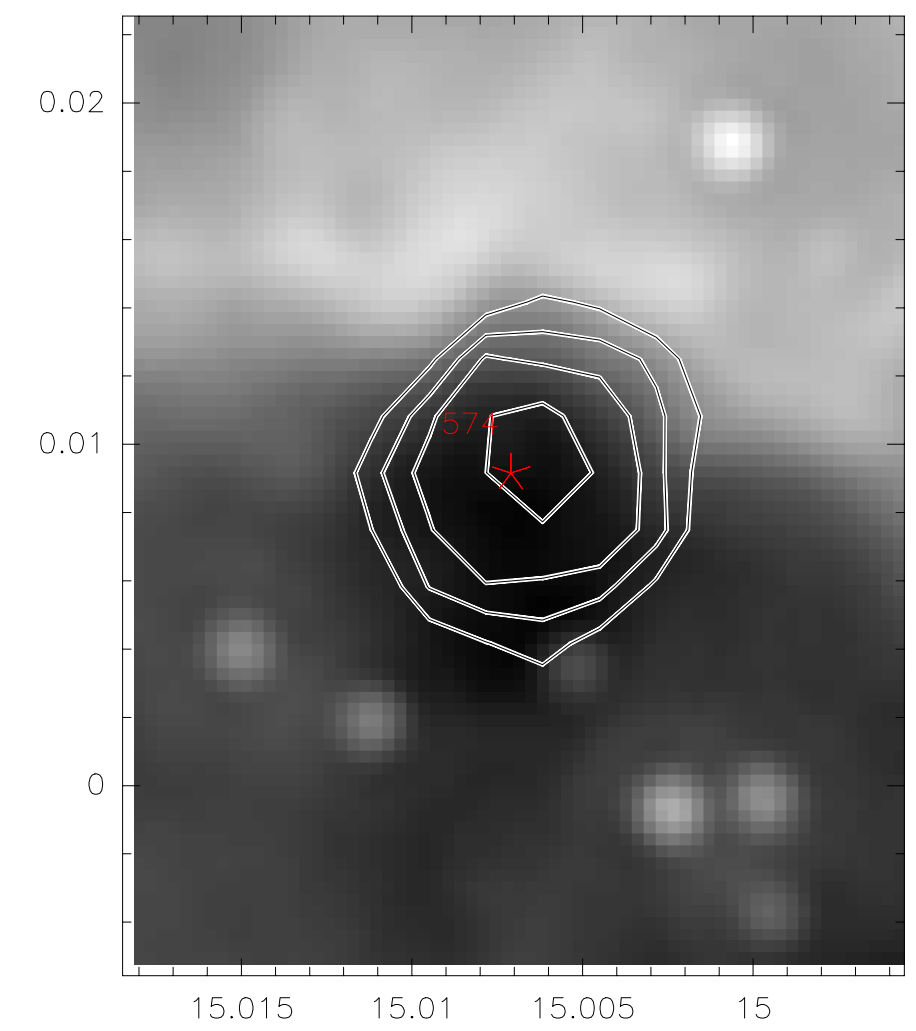

Fig. C.1. continued.

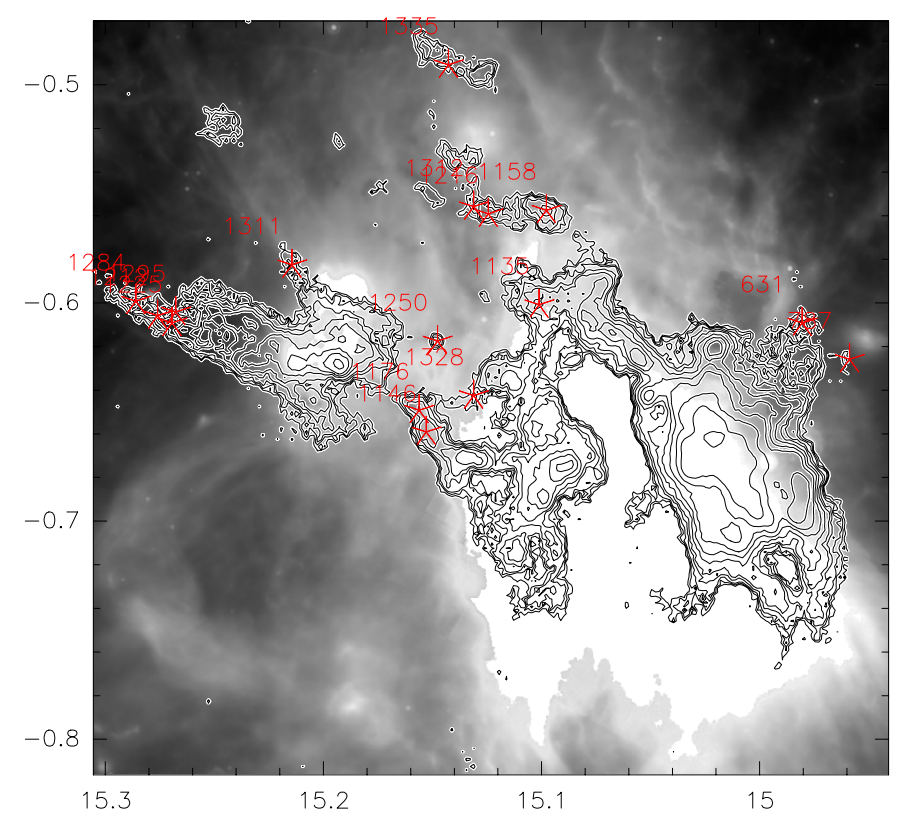

Fig. C.1. continued. 
J. Tackenberg et al.: Starless clumps in ATLASGAL
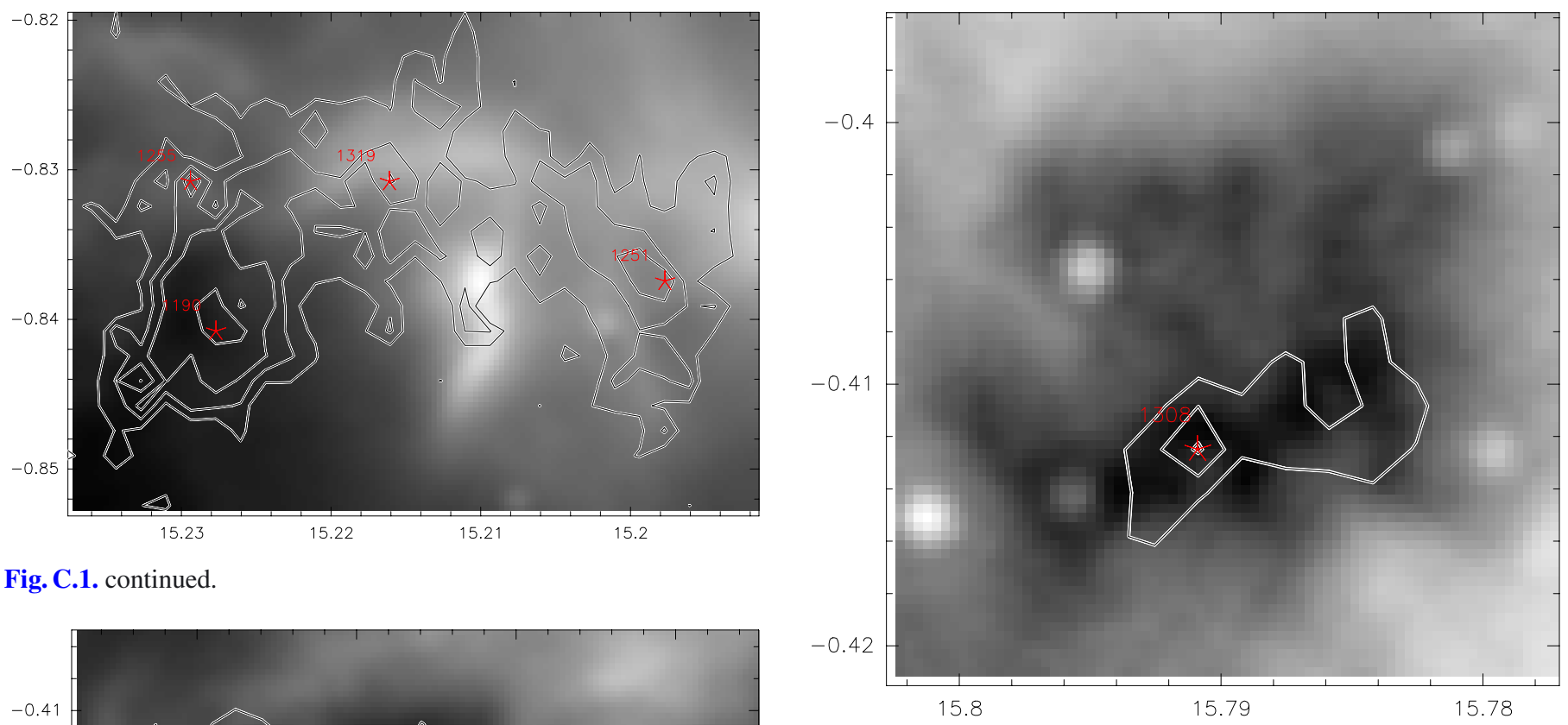

Fig. C.1. continued.

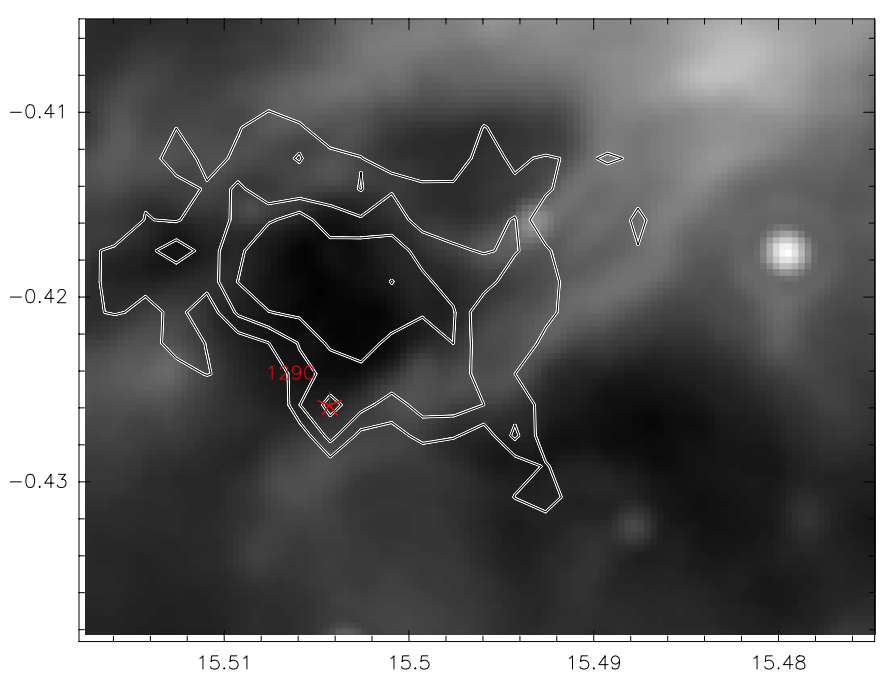

Fig. C.1. continued.

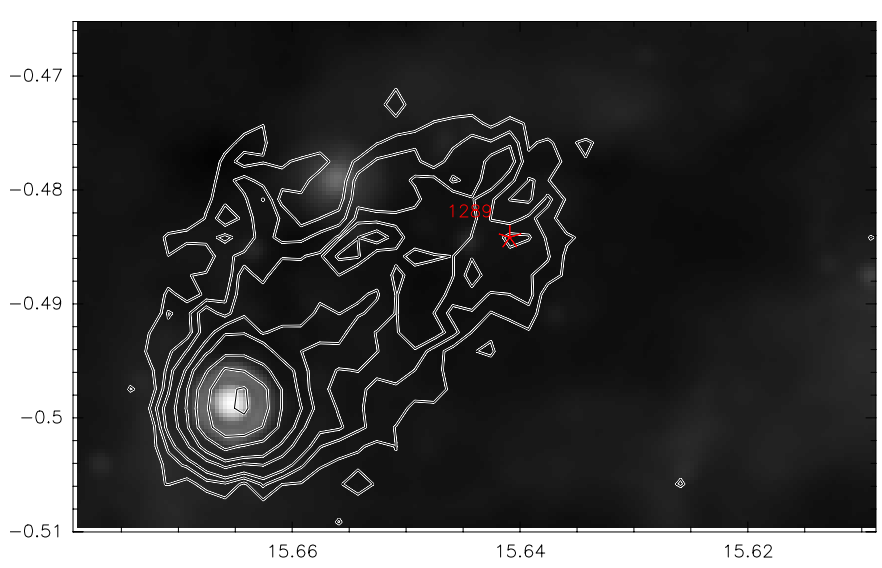

Fig. C.1. continued.

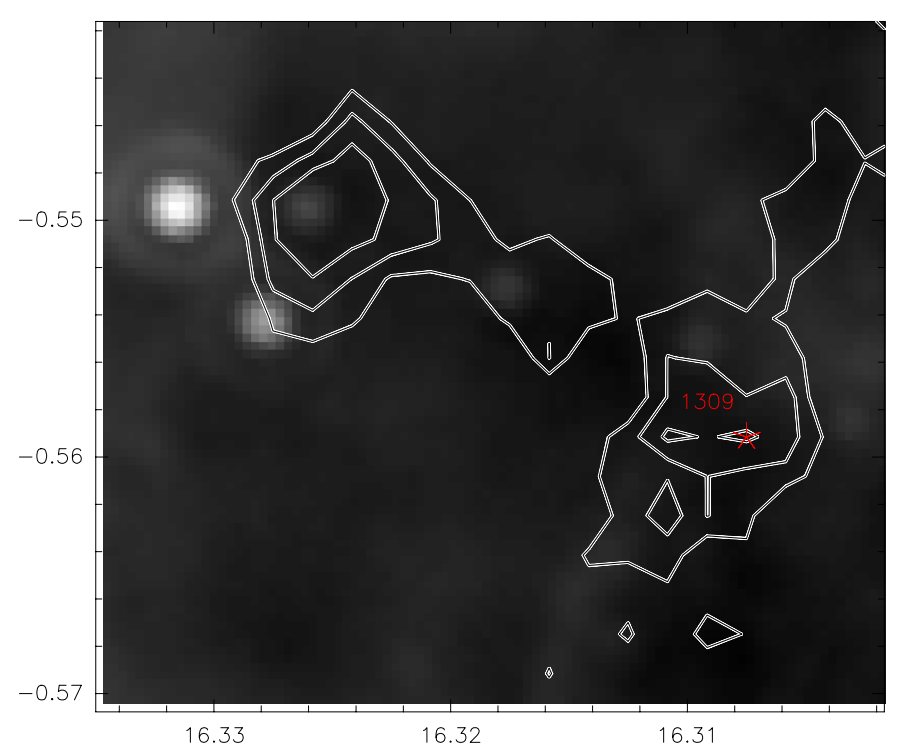

Fig. C.1. continued.

Fig. C.1. continued. 
A\&A 540, A113 (2012)

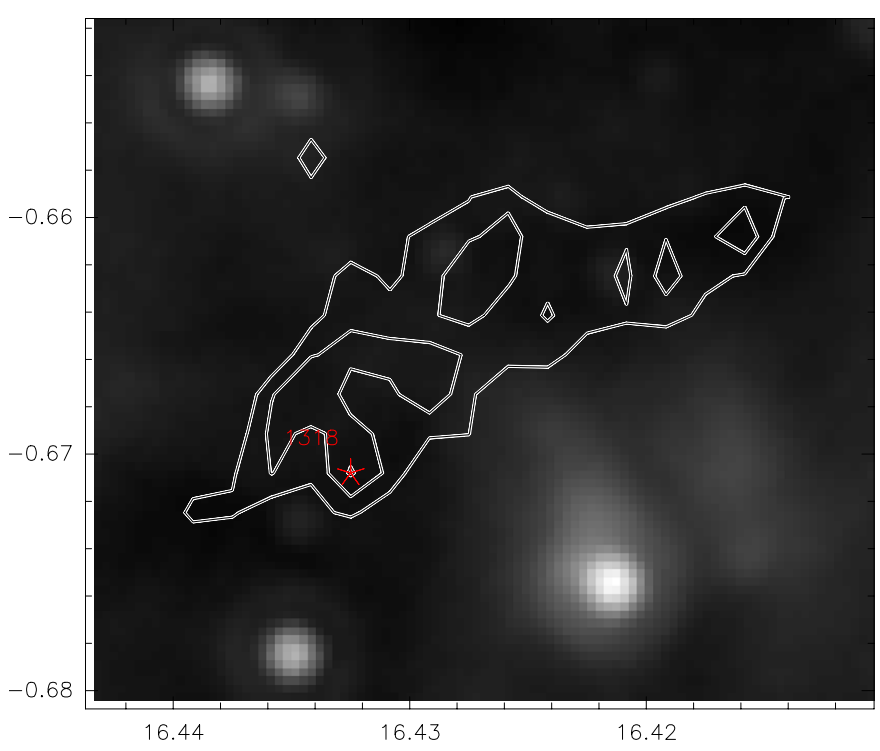

Fig. C.1. continued.

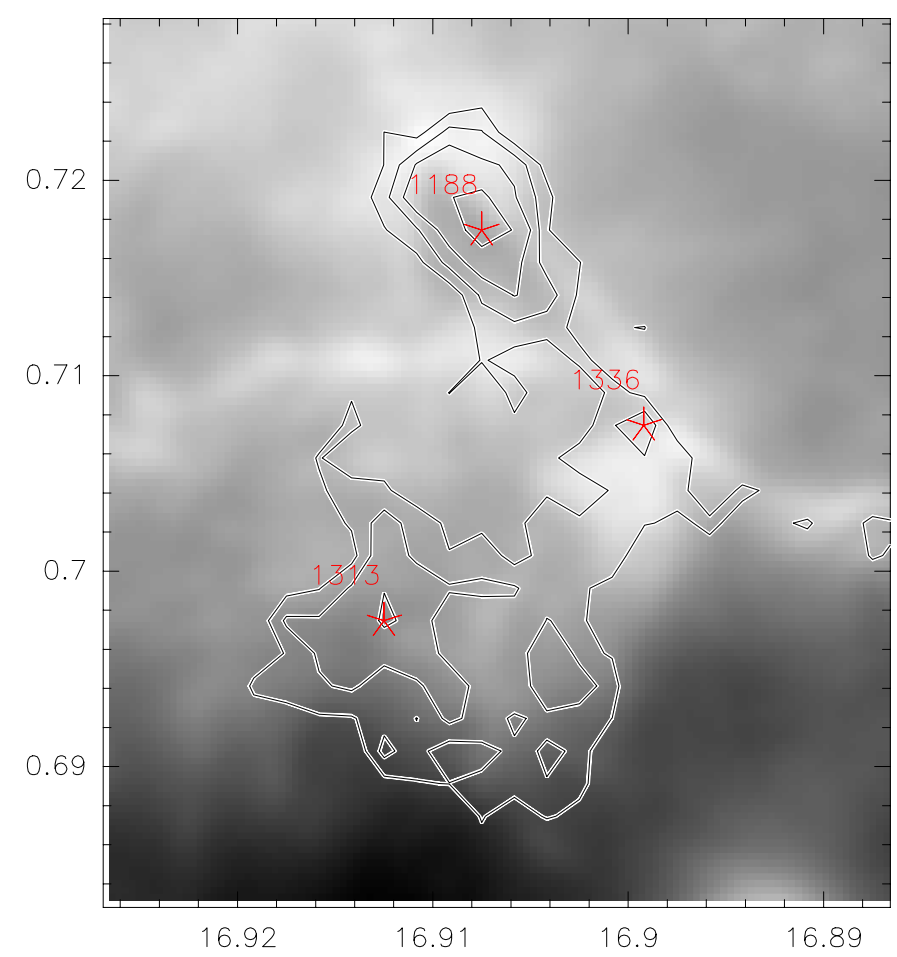

Fig. C.1. continued.

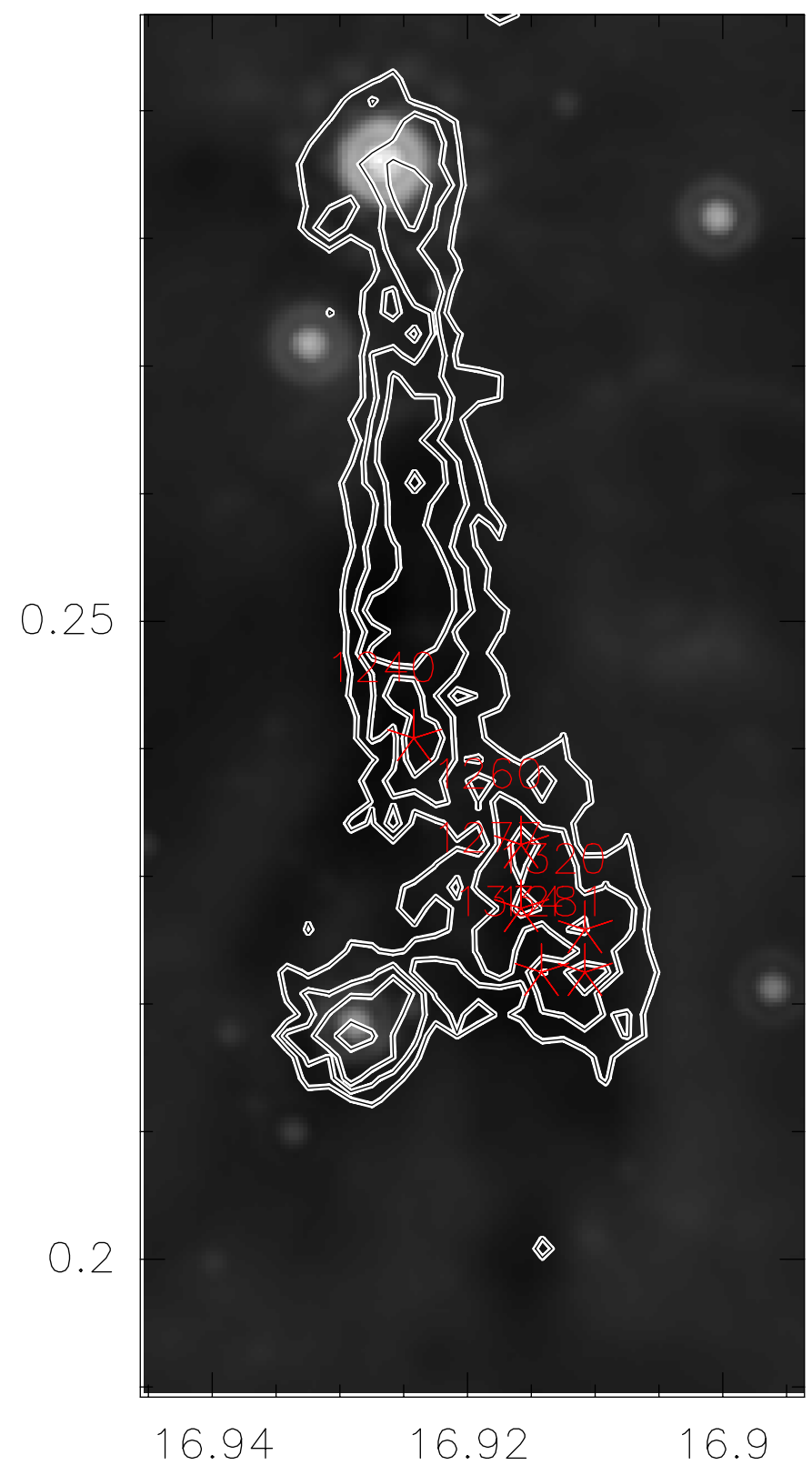

Fig. C.1. continued. 
J. Tackenberg et al.: Starless clumps in ATLASGAL

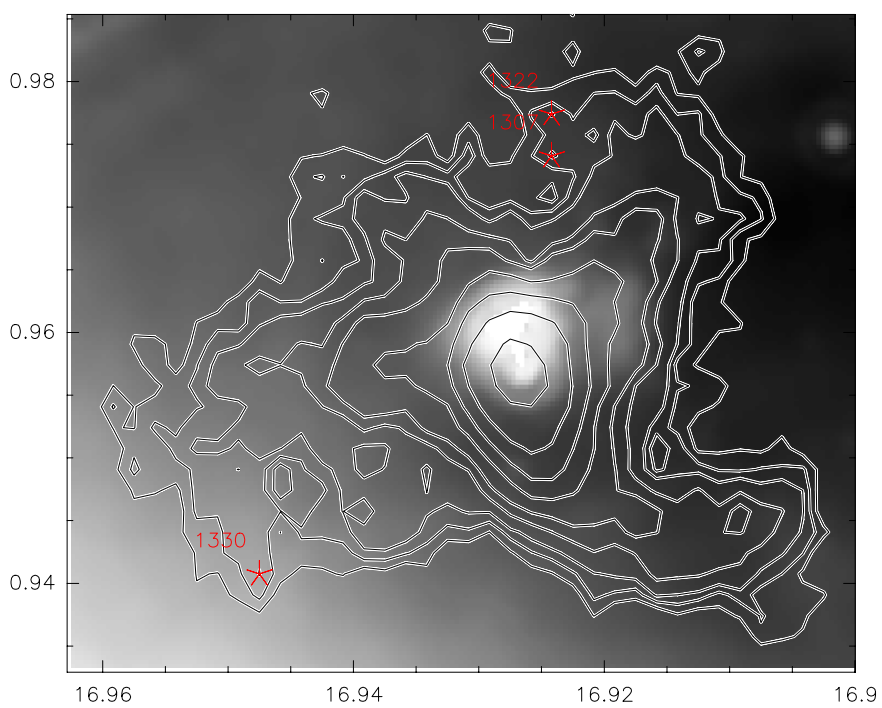

Fig. C.1. continued.
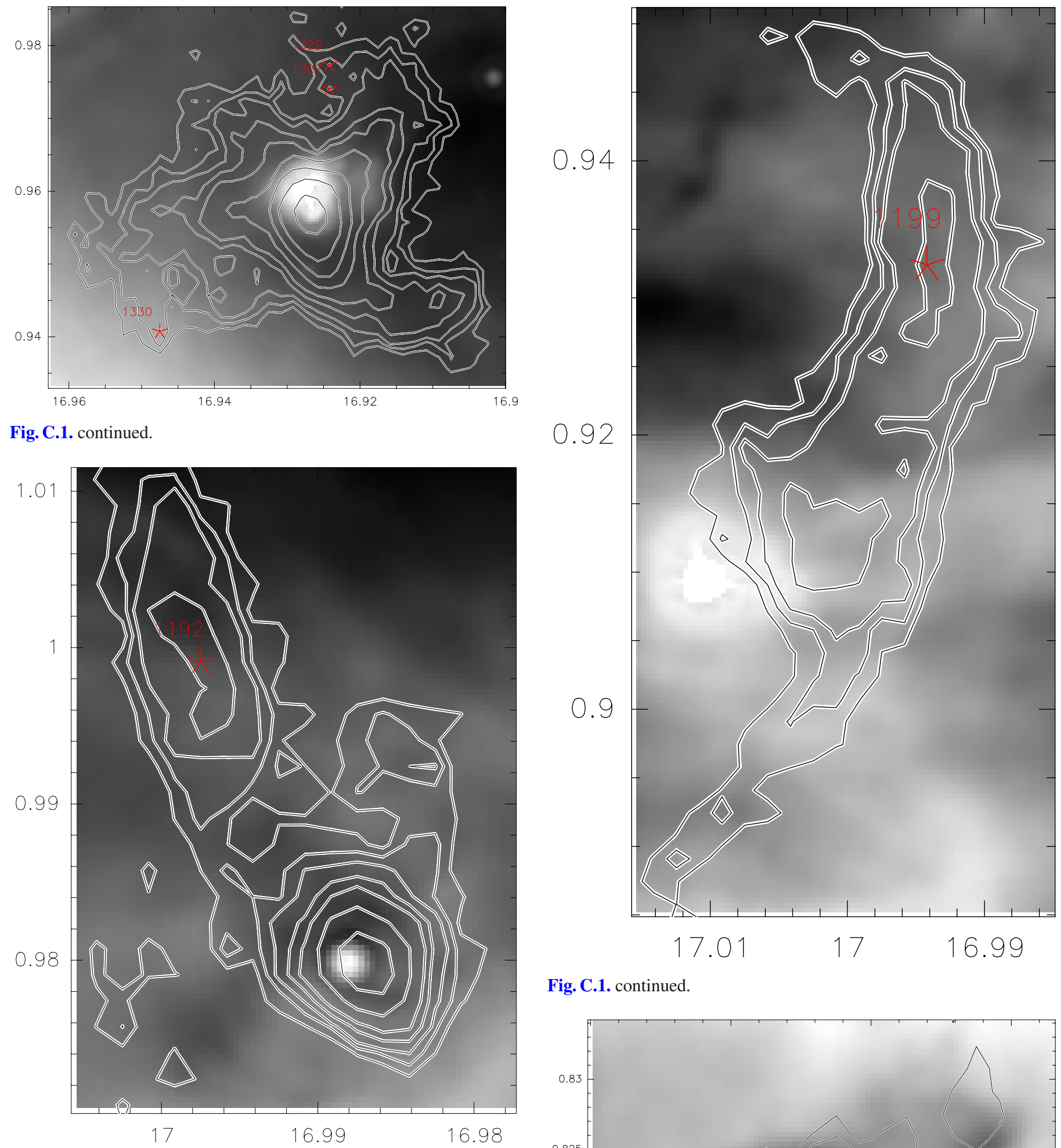

Fig. C.1. continued.

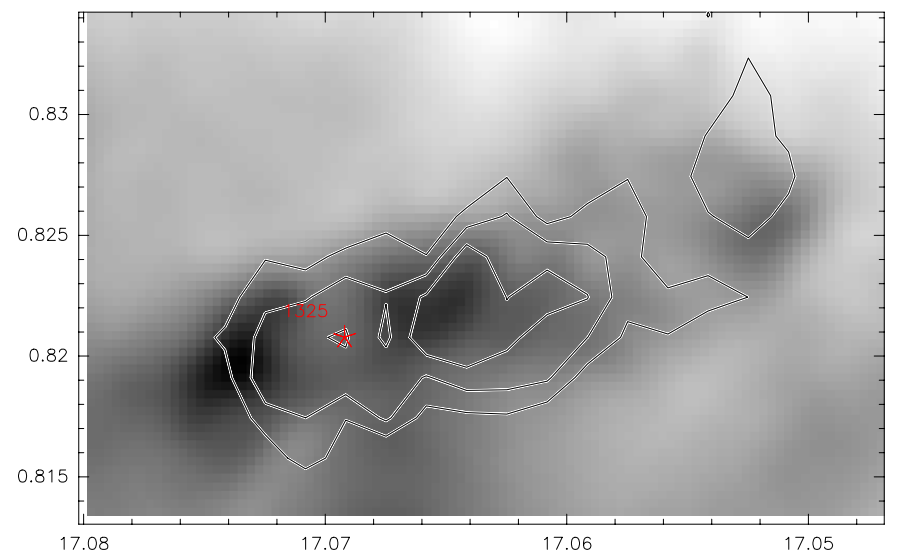

Fig. C.1. continued. 
A\&A 540, A113 (2012)

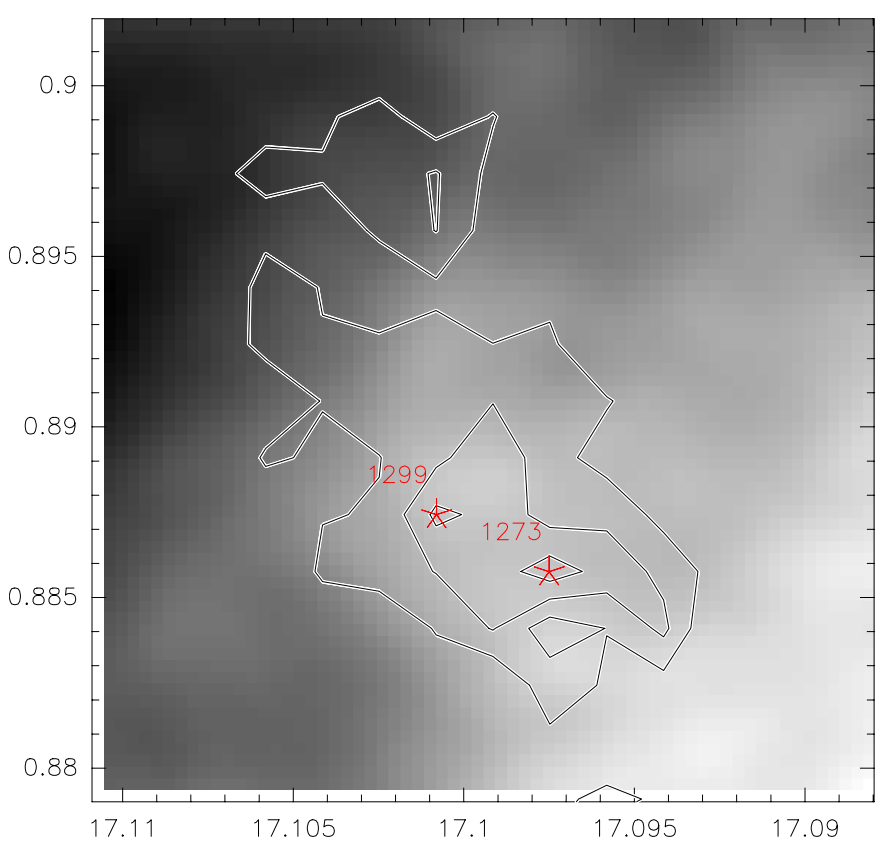

Fig. C.1. continued.

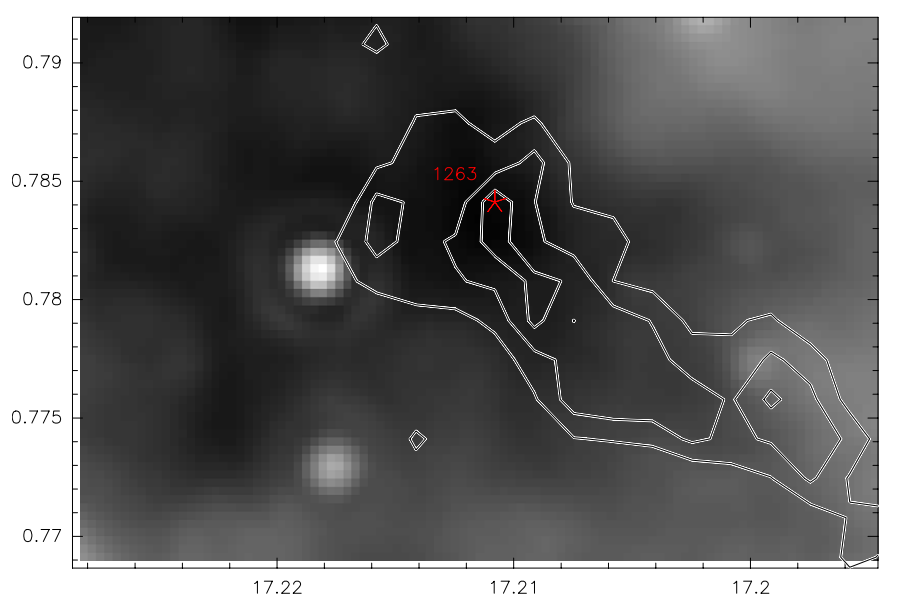

Fig. C.1. continued.

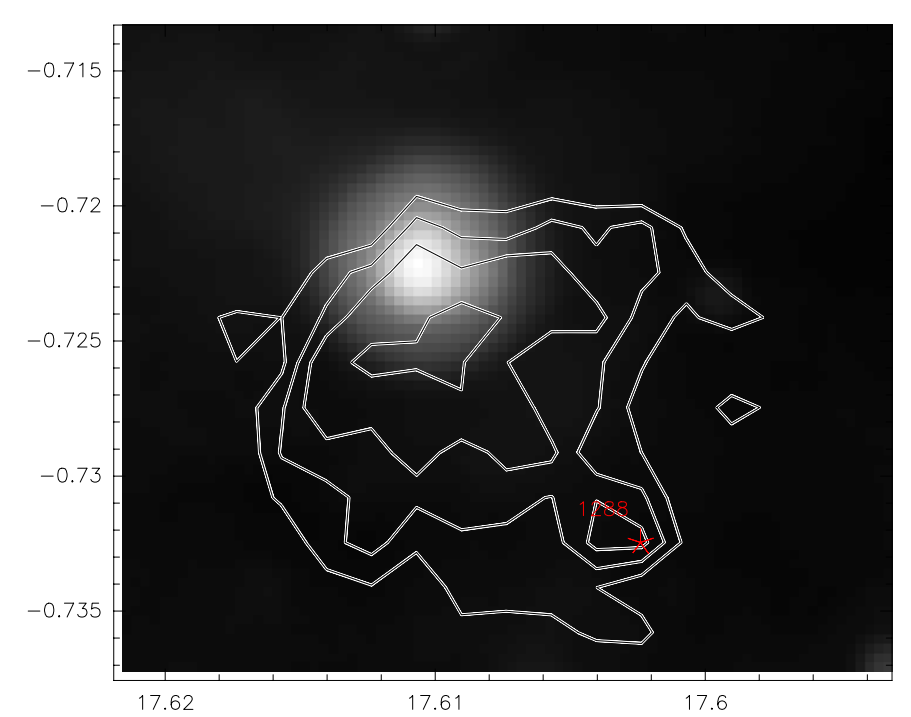

Fig. C.1. continued.

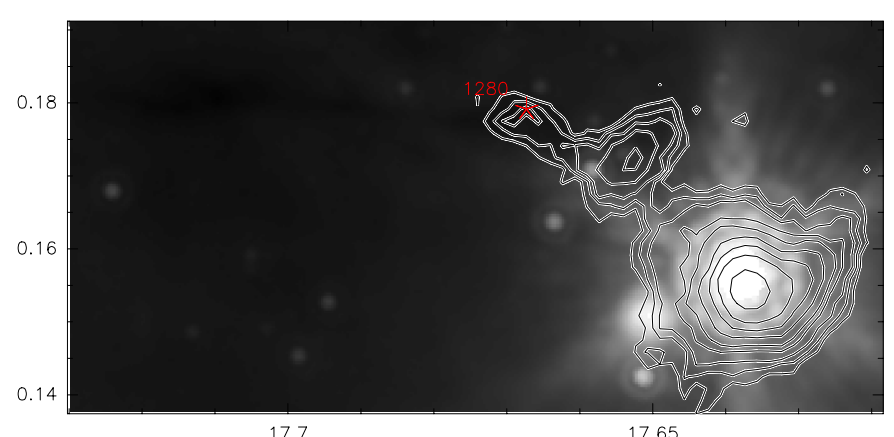

Fig. C.1. continued.

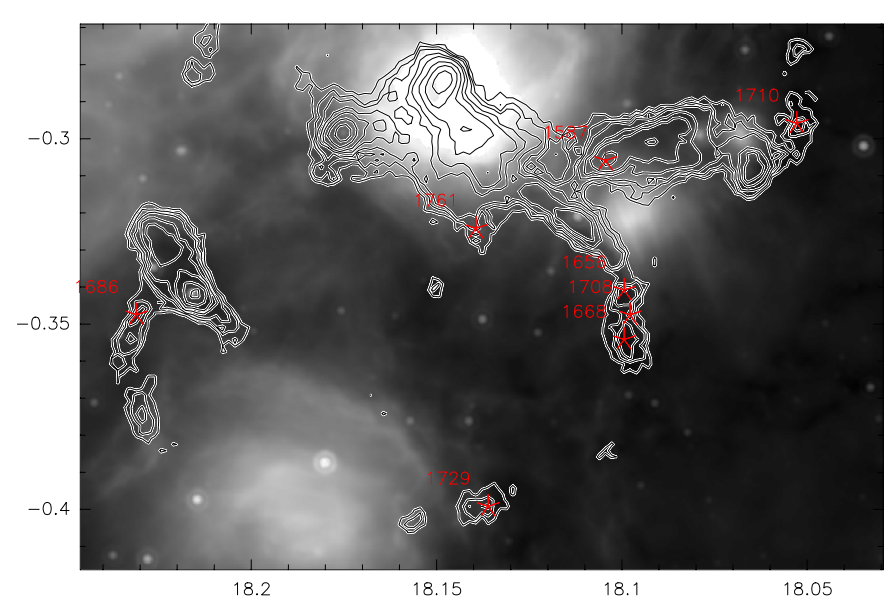

Fig. C.1. continued.

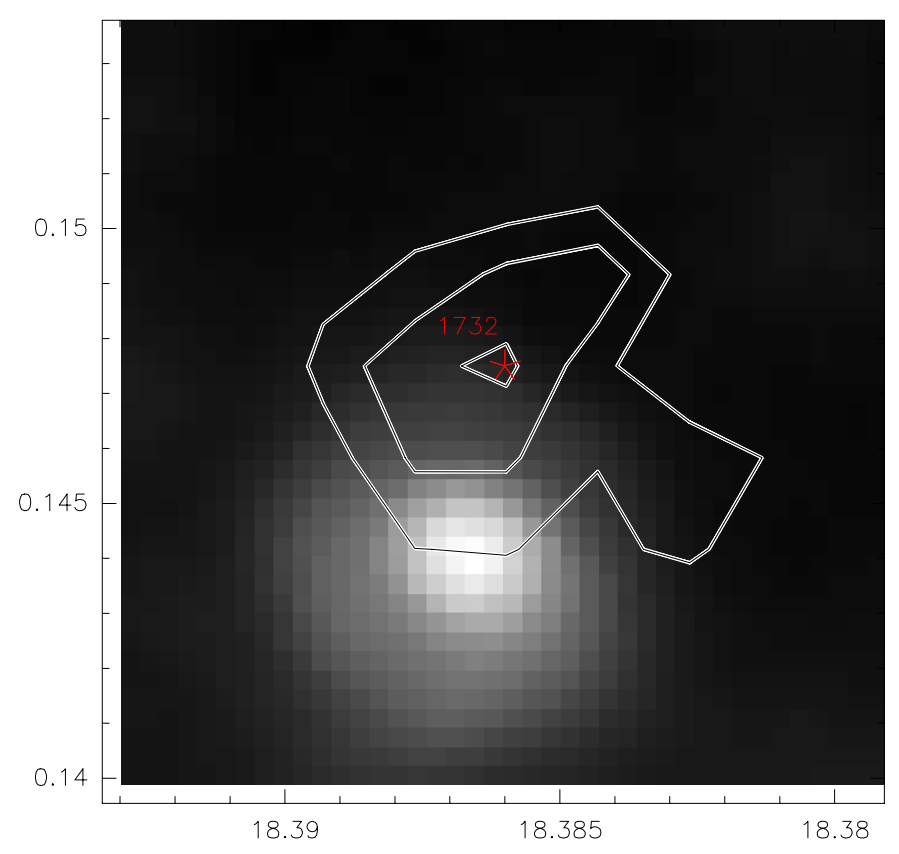

Fig. C.1. continued. 
J. Tackenberg et al.: Starless clumps in ATLASGAL
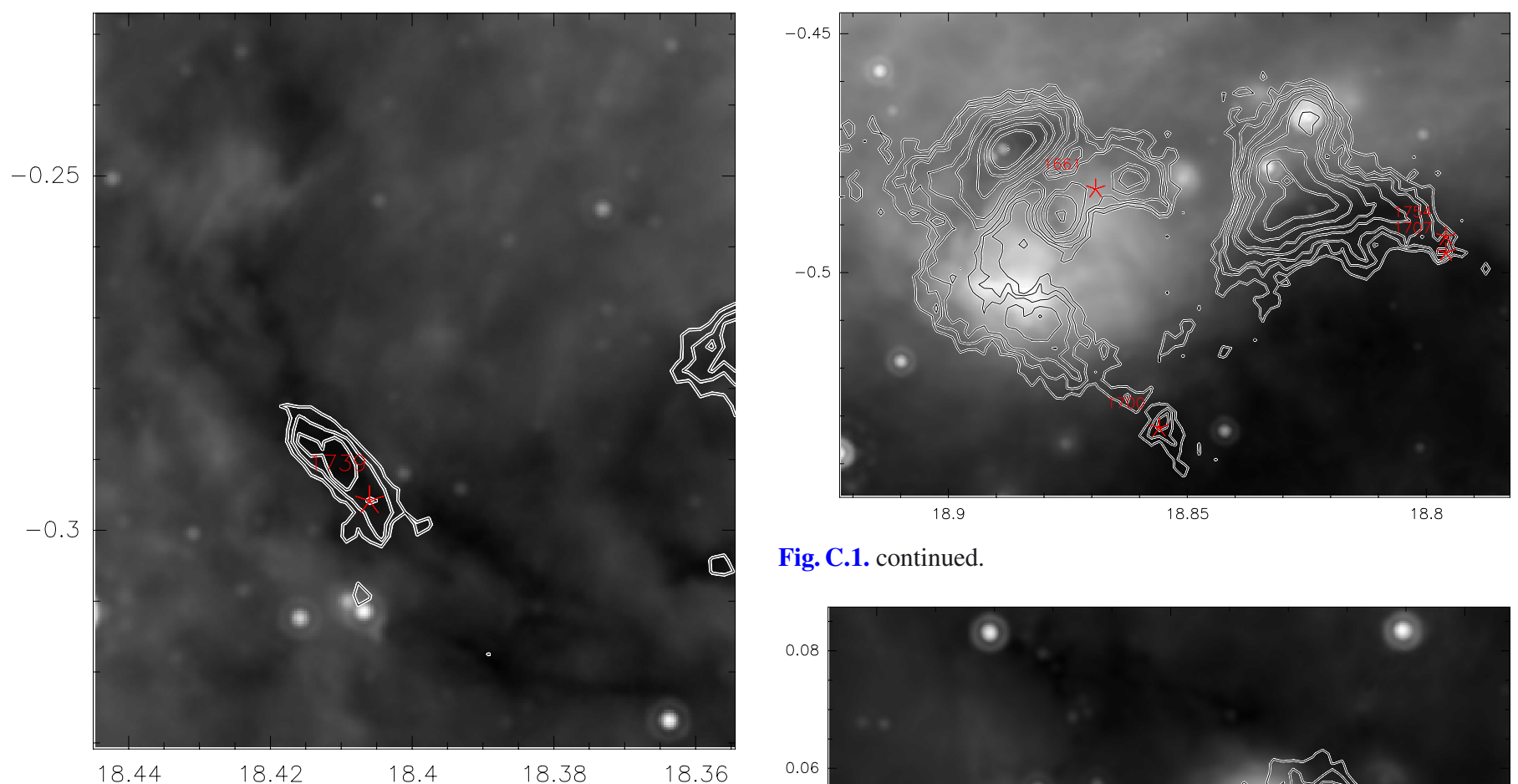

Fig. C.1. continued.

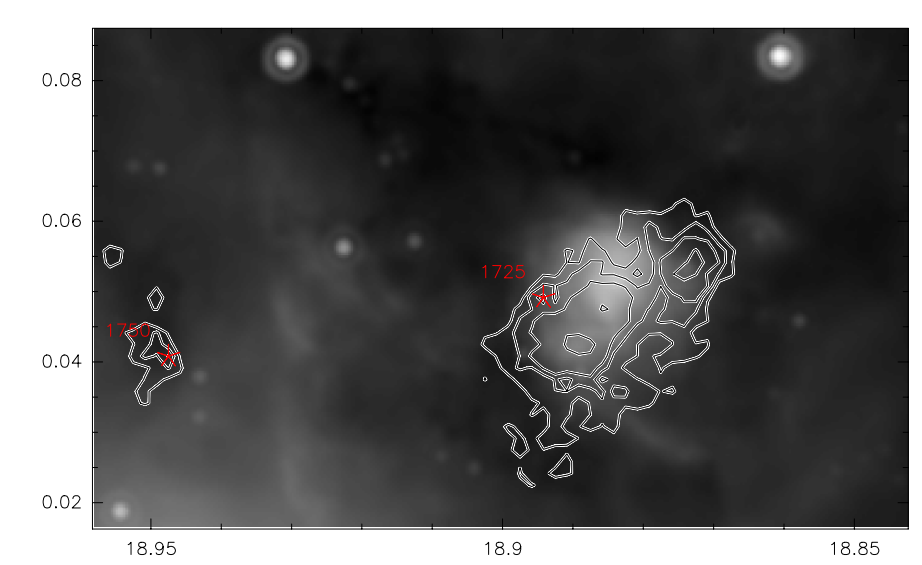

Fig. C.1. continued.

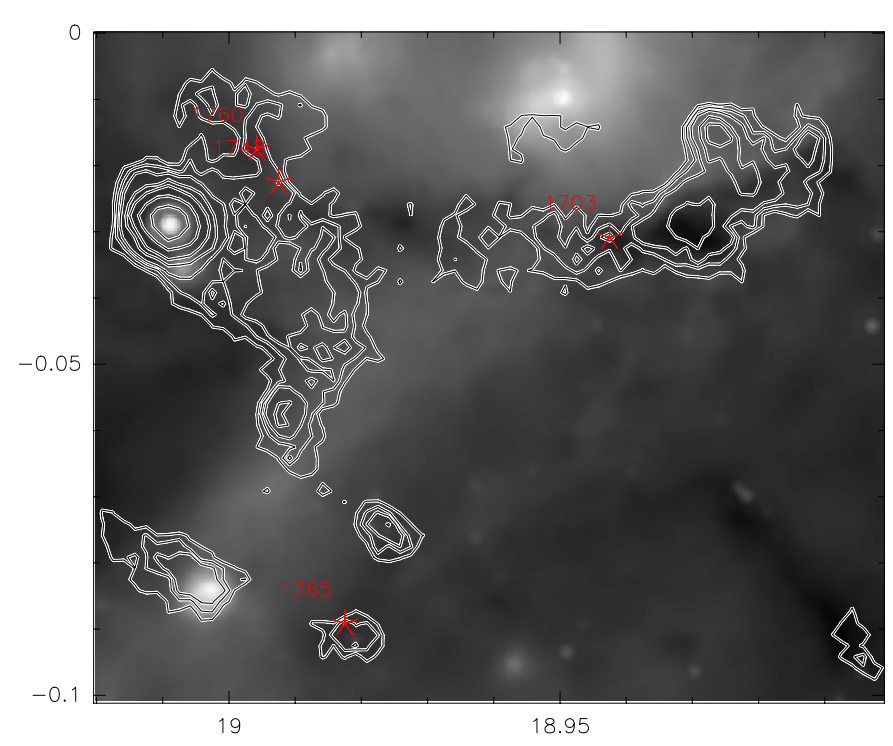

Fig. C.1. continued. 
A\&A 540, A113 (2012)

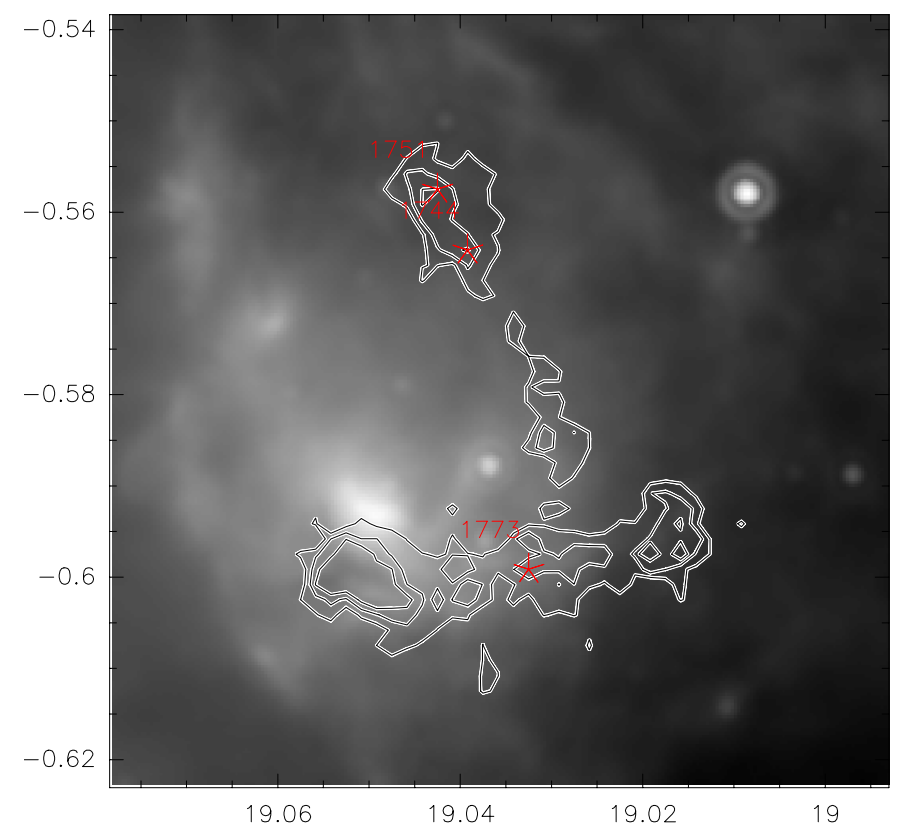

Fig. C.1. continued.

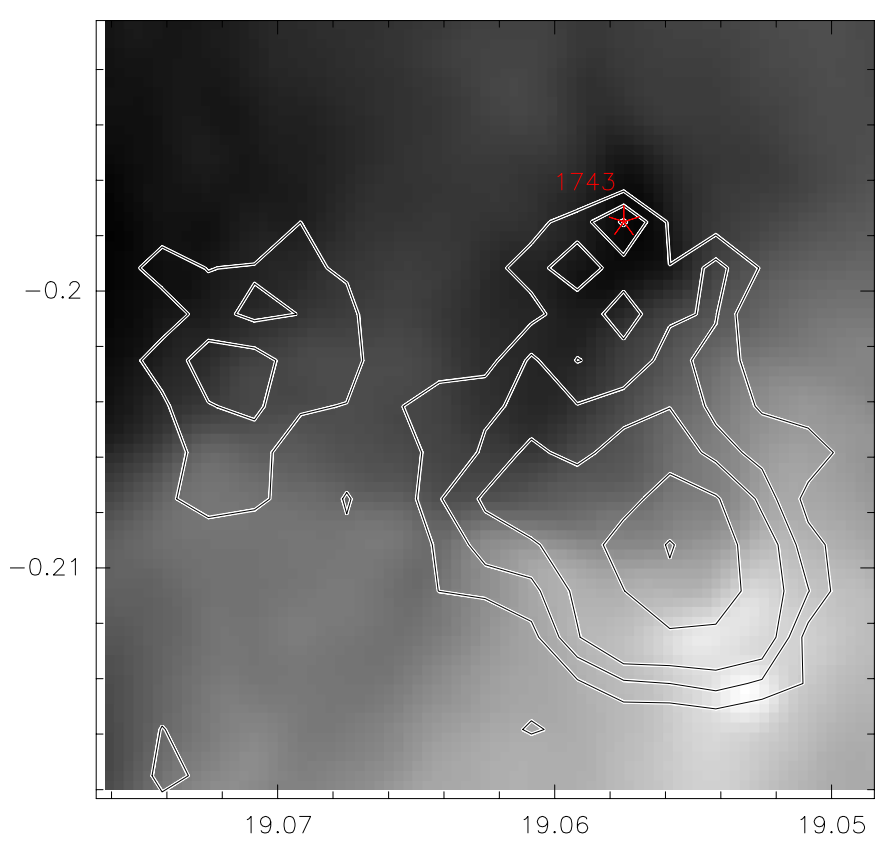

Fig. C.1. continued.

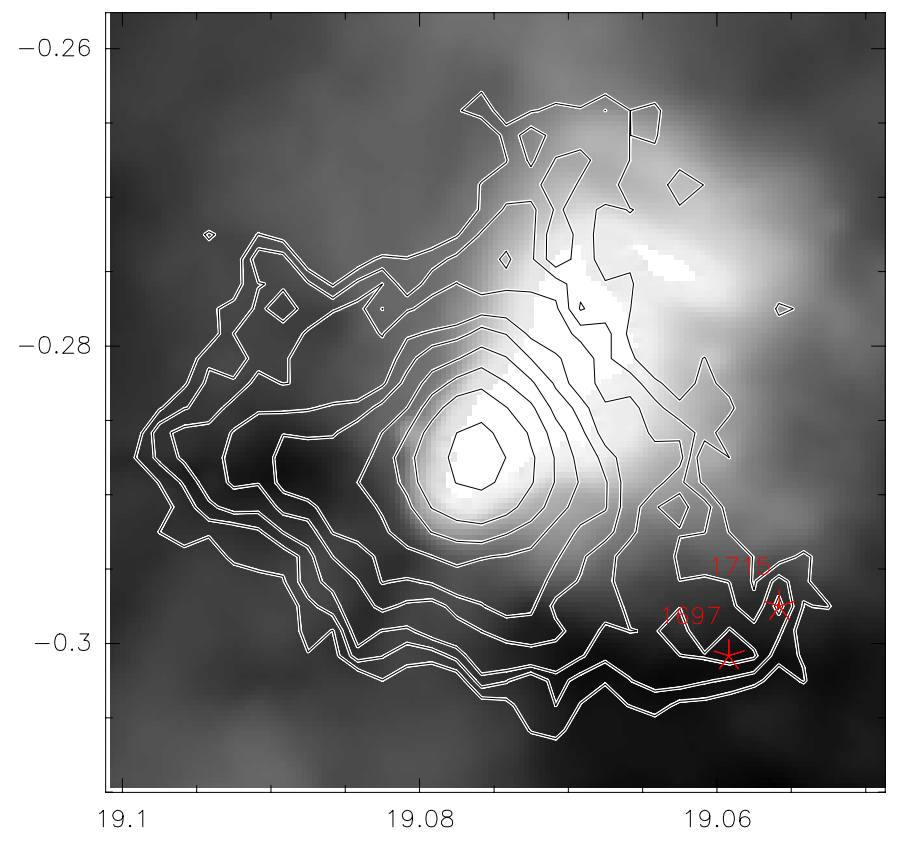

Fig. C.1. continued.

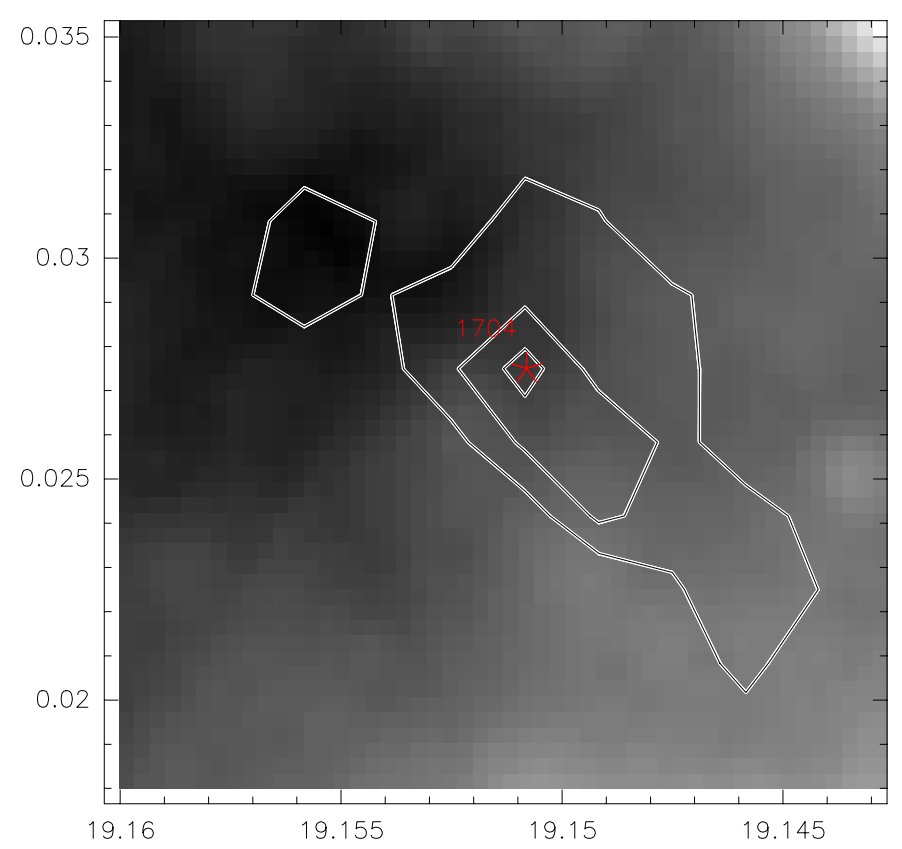

Fig. C.1. continued.

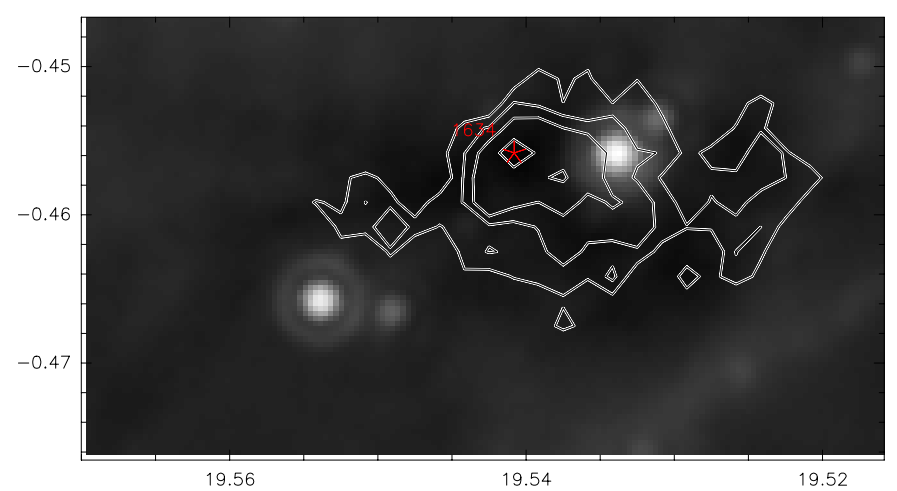

Fig. C.1. continued. 
J. Tackenberg et al.: Starless clumps in ATLASGAL
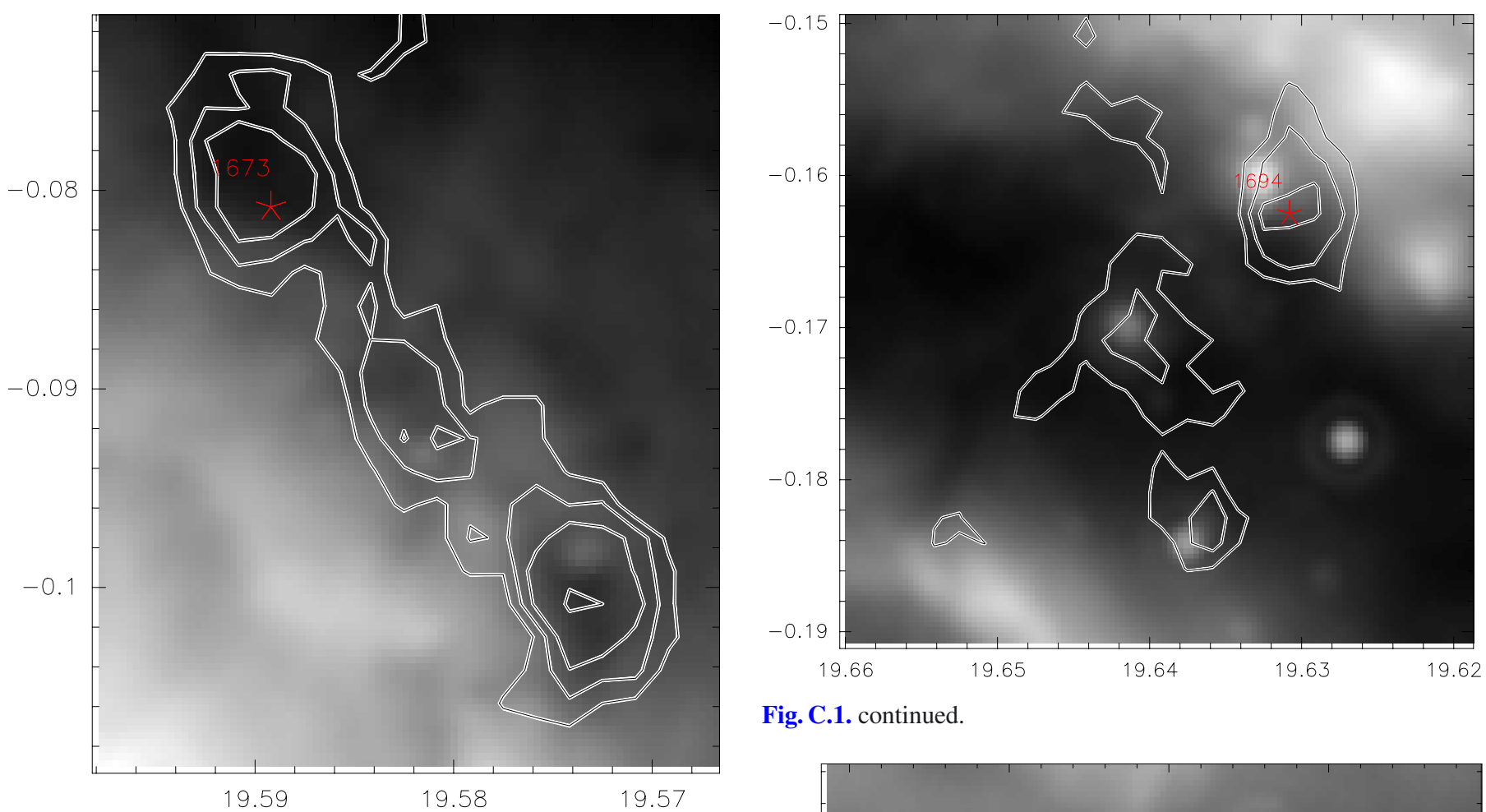

Fig. C.1. continued.

Fig. C.1. continued.
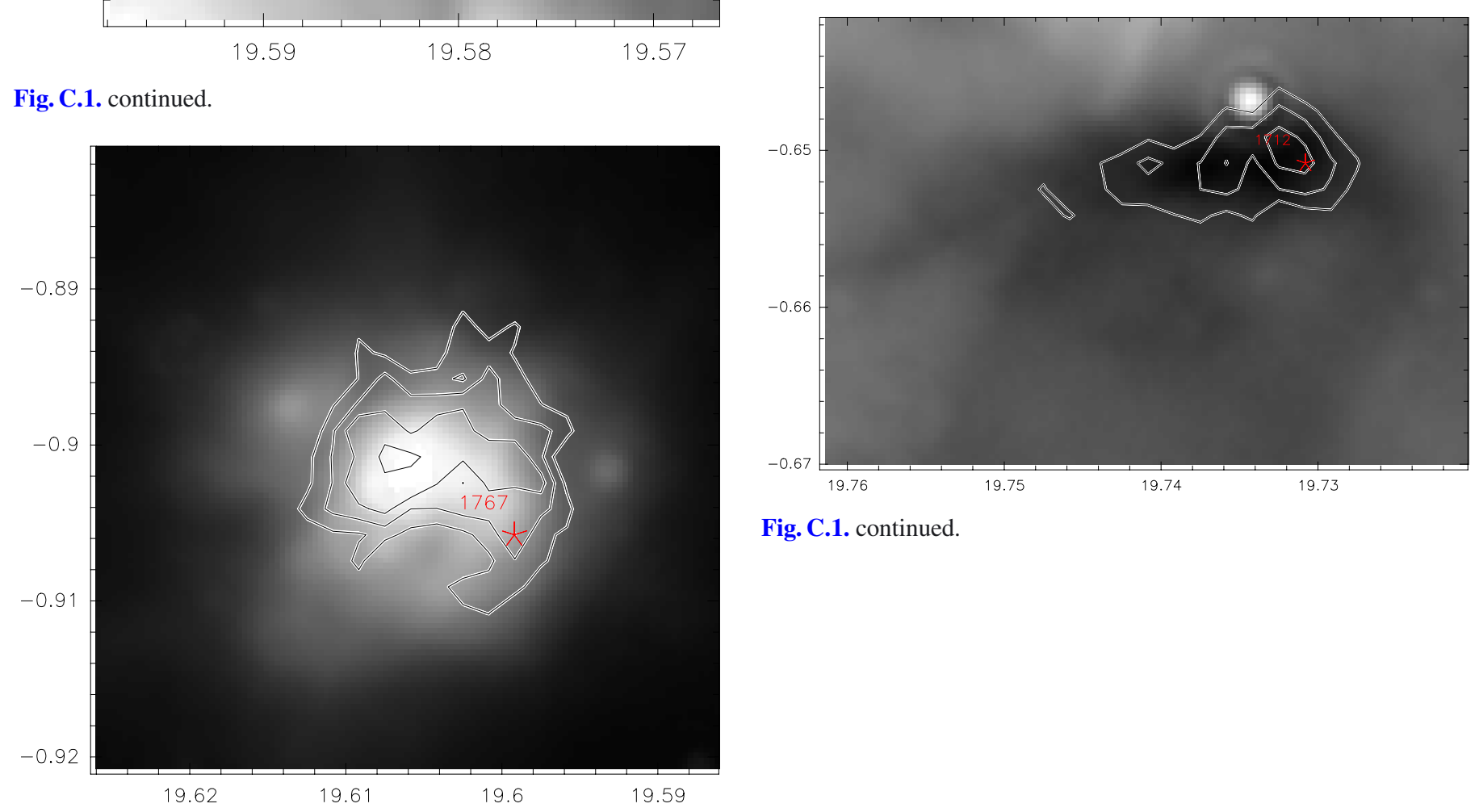

Fig. C.1. continued.

Fig. C.1. continued. 
A\&A 540, A113 (2012)

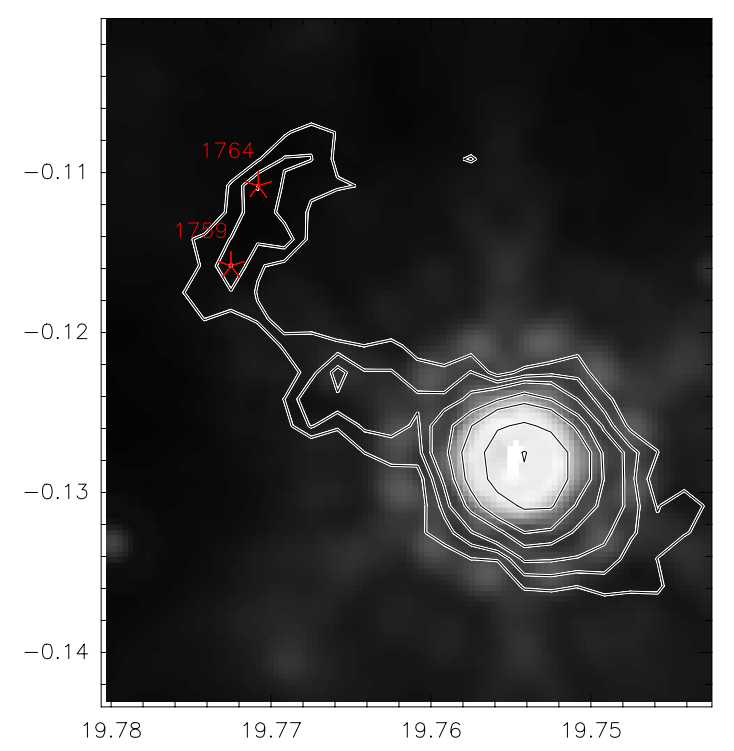

Fig. C.1. continued.

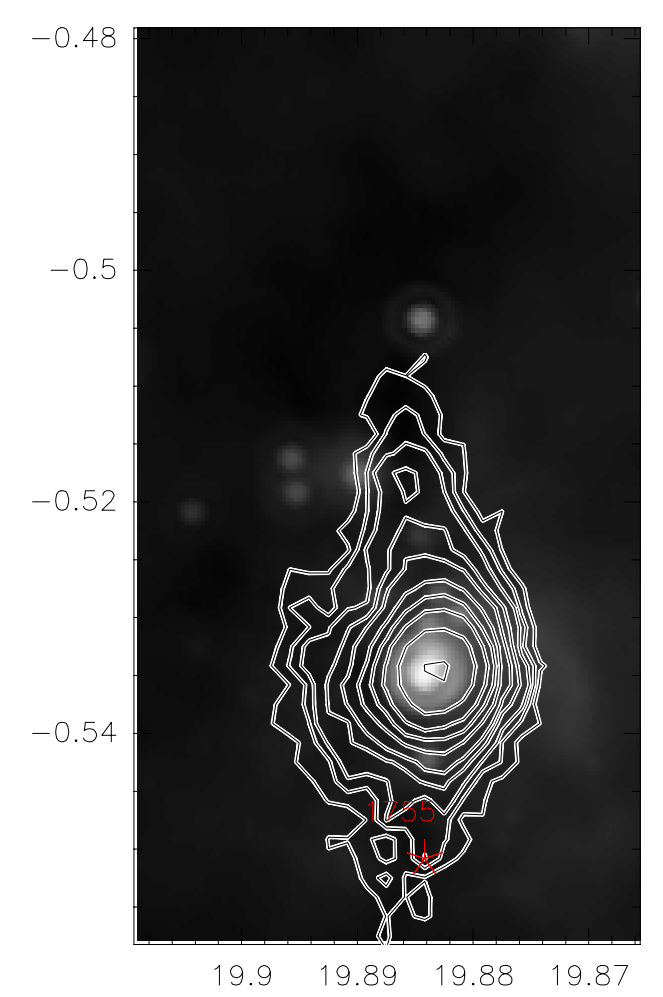

Fig. C.1. continued. 